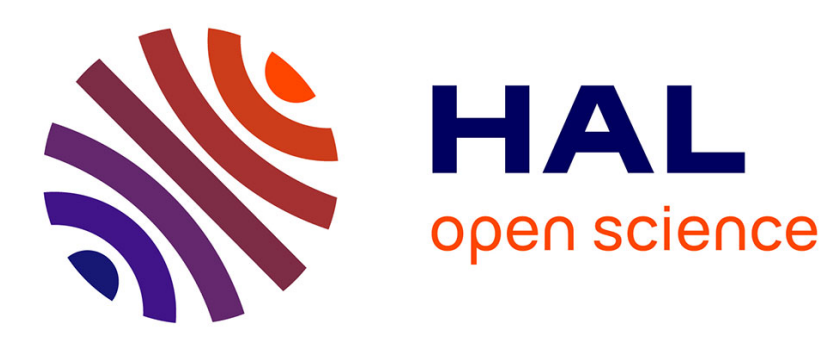

\title{
WKB expansions for weakly well-posed hyperbolic boundary value problems in a strip: time depending loss of derivatives
}

Antoine Benoit

\section{To cite this version:}

Antoine Benoit. WKB expansions for weakly well-posed hyperbolic boundary value problems in a strip: time depending loss of derivatives. 2019. hal-02391809

\section{HAL Id: hal-02391809 \\ https://hal.science/hal-02391809}

Preprint submitted on 3 Dec 2019

HAL is a multi-disciplinary open access archive for the deposit and dissemination of scientific research documents, whether they are published or not. The documents may come from teaching and research institutions in France or abroad, or from public or private research centers.
L'archive ouverte pluridisciplinaire HAL, est destinée au dépôt et à la diffusion de documents scientifiques de niveau recherche, publiés ou non, émanant des établissements d'enseignement et de recherche français ou étrangers, des laboratoires publics ou privés. 


\title{
WKB expansions for weakly well-posed hyperbolic boundary value problems in a strip: time depending loss of derivatives
}

\author{
Antoine BENOIT \\ Université du Littoral Côte d'Opale, LMPA \\ 50 rue Ferdinand Buisson CS 8069962228 Calais, France \\ Email: antoine.benoit@univ-littoral.fr
}

Phone: +333 21463651

December 3, 2019

\begin{abstract}
In this article we are interested in linear hyperbolic systems of equations defined in the strip $\mathbb{R} \times] 0,1[$. More precisely the aim of this article is to described the influence of the boundary conditions on the behaviour of the solution. This question has already been adressed in Benb in which the author shows that in the strip geometry, the self-interaction (meaning that a wave packet regenerates itself by repeated rebounds against the sides of the strip) phenomenon becomes generic and can lead to some exponential growth in time of the solution.

Here we restrict our attention to finite time problems (so that we are not interested in the possible growth in time of the solution) however the main difficulty is that we do not require that the boundary conditions lead to strongly well-posed problems but only to weakly well-posed problems (that is loss(es) of derivatives are possible).

The question is thus to determine what can be the minimal loss of derivatives in the energy estimate of the solution.

The main result of this article is to show, thanks to geometric optics expansions, that if the strip problem admits a boundary in the so-called $W R$-class of BGRSZ02 then the loss of derivatives shall be at least increasing with the time of resolution. More precisely this loss is bounded by below by a step function increasing with respect to time which depends on the minimal time needed to perform a full regeneration of the wave packet.
\end{abstract}

AMS subject classification: 35L04 78A05

Key words: hyperbolic boundary value problems, weak well-posedness, geometric optics expansions, strip geometry, self-interaction phenomenon.

Acknowledgements: The author acknowledge financial support from the ANR research project NABUCO.

\section{Contents}

1 Introduction 2

2 Formal discussion $\mathbf{4}$

$2.1 \quad$ Formal justification for $N=2 \ldots \ldots$. . . . . . . . . . . . . . . . . . . . . . . . . . . 4

2.2 Extension to the framework $N>2 \ldots \ldots \ldots \ldots$

3 Notations and main results 10

3.1 Notations and assumptions . . . . . . . . . . . . . . . . . . . . . . . 10

3.1.1 About the operator $L(\partial) \quad \ldots \ldots \ldots$. . . . . . . . . . . . . . . . . . . . . . . 10

3.1 .2 About the boundary conditions . . . . . . . . . . . . . . . . . . . . . . . 12

3.2 Main result and proof . . . . . . . . . . . . . . . . . . . . . 13 
3.2.1 Statement . . . . . . . . . . . . . . . . . . . . . . . . 13

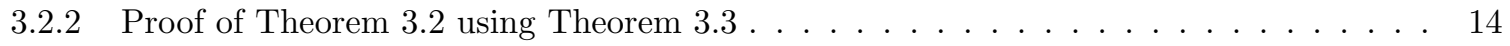

4 Construction of the geometric optics expansion 15

4.1 Useful notations for geometric optics expansions . . . . . . . . . . . . . . . . . . . . . . . . . 15

4.2 The cascades of equations . . . . . . . . . . . . . . . . . . . . . . . . 15

4.3 Determination of the leading order amplitudes . . . . . . . . . . . . . . . . . . . . . . . . 16

4.3 .1 Determination of $\varrho_{0}^{\varepsilon} \ldots \ldots \ldots \ldots \ldots$

4.3.2 The general equation on $\varrho_{n}^{\varepsilon}, \varrho_{n+1}^{\varepsilon}, \nu_{n}^{\varepsilon}, \nu_{n+1}^{\varepsilon}$ for $n \geq 1$. . . . . . . . . . . . . 23

4.3.3 The explicit expression of $\varrho_{0}^{\varepsilon}$, end of the determination of the leading order amplitudes 34

4.4 Determination of higher order amplitudes and summary . . . . . . . . . . . . . . . . 34

5 Examples and comments 36

5.1 Examples . . . . . . . . . . . . . . . . . . . . . . . . . . 36

5.2 Conclusion and comments . . . . . . . . . . . . . . . . . . . . . . . . 37

\section{Introduction}

In this article we study geometric optics expansions for the following hyperbolic system of partial differential equations set in a two dimensional strip. With more details for some $T>0$ we consider the system:

$$
\begin{cases}L(\partial) u:=\partial_{t} u+A_{1} \partial_{1} u+A_{2} \partial_{2} u=f & \text { for } \left.\left.\left.\left(t, x_{1}, x_{2}\right) \in\right]-\infty, T\right] \times \mathbb{R} \times\right] 0,1\left[:=\Omega_{T}=\Omega,\right. \\ B_{0} u_{\mid x_{2}=0}=g_{0} & \text { for } \left.\left.\left(t, x_{1}\right) \in\right]-\infty, T\right] \times \mathbb{R}:=\partial \Omega_{0, T}=\partial \Omega_{0}, \\ B_{1} u_{\mid x_{2}=1}=g_{1} & \text { for } \left.\left.\left(t, x_{1}\right) \in\right]-\infty, T\right] \times \mathbb{R}:=\partial \Omega_{1, T}=\partial \Omega_{1}, \\ u_{\mid t \leq 0}=0 & \text { for } \left.\left(x_{1}, x_{2}\right) \in \mathbb{R} \times\right] 0,1[:=\Gamma,\end{cases}
$$

where for $N \in \mathbb{N}$, the matrices $A_{j} \in \mathbf{M}_{N \times N}(\mathbb{R})$ and where the boundary matrix $B_{0}$ (resp. $B_{1}$ ) lies in $\mathbf{M}_{p_{0} \times N}(\mathbb{R})$ (resp. $\mathbf{M}_{p_{1} \times N}(\mathbb{R})$ ). We assume that $p_{0}$ and $p_{1}$ are the good numbers of boundary conditions specified in Assumption 3.2

This article is mainly devoted to the construction of geometric optics expansions (also called $W K B$ expansions) for (1), that is the construction of an approximate solution of (1). This construction is performed in the particular setting of weak well-posedness (more precisely for systems in the so-called $W R$-class (for " Weakly Real") see [BGRSZ02]) in order to investigate what are the possible losses of regularity (or derivative) of the solution compared to the data of the problem.

Such constructions of geometric optics expansions in this context have a long story starting (in the author's knowledge) by the formal constructions given by [AM87] and MR83] (see also [Maj88]) to explain the appearance of singularities in (non linear) fluid dynamics such as Mach stems formation. We also refer to CW17. (and the many references therein) for the construction of such rigorous geometric optics expansions.

Geometric optics expansions aim to construct an approximate solution of (1) when the source terms are highly oscillating, and if the approximate solution is "close enough" of the exact solution to highlight some phenomena on the exact solution of (1) from the approximate one.

Consider in (1) that $f \equiv 0, g_{1} \equiv 0$, so that the only non trivial source term is $g_{0}$ and it reads under the form: for $0<\varepsilon \ll 1$,

$$
g_{0}\left(t, x_{1}\right):=g_{0}^{\varepsilon}\left(t, x_{1}\right)=e^{\frac{i}{\varepsilon} \varphi\left(t, x_{1}\right)} g\left(t, x_{1}\right),
$$

where $\varphi$ is a linear phase function and where the amplitude $g$ is assumed to be sufficiently smooth and vanishes for negative time.

In the high frequency regime, such a source term generically generates waves packets travelling from the side $\partial \Omega_{0}$ to the interior of the domain. In the more classical setting of the half-space geometry these waves spread to infinity, they will never be reflected back, so the situation is simpler and well-understood. 
However the same behaviour does not hold anymore if one considers more complex geometries like the strip [Benb] because some extra waves coming from the possible reflections against the second component of the boundary (namely the set $\left\{x_{2}=1\right\}$ for the strip geometry) have to be considered in the geometric optics expansion. These extra phases give raise to self-interaction phenomenon which have already been discussed in SS75] or Benb. By self-interaction we mean that a wave packet can regenerate itself after several reflections against the different components of the boundary.

This possible self-interaction between the different waves implies that in order to initialize the construction of the $W K B$ expansion a new invertibility condition has to be imposed. This condition seem $\$$ to be linked with a maximal growth of the energy during the repeated reflections. The growth of the solution during a reflection is given by the so-called uniform Kreiss-Lopatinskii inverse.

Indeed, it is known from the seminal works of Kreiss [Kre70] that the strong well-posedness of the problem in the half-space (in the sense that we have existence, uniqueness of the solution and that this solution is as regular as the source terms of the problem (in some weighted in time $L^{2}$-norm)) is equivalent to the uniform Kreiss-Lopatinskii condition. This condition means that in the normal mode analysis no stable mode is solution of the homogeneous boundary condition.

More precisely it means that if $\zeta$ is a frequency parameter then for all $\zeta$ if $E^{s}(\zeta)$ stands for the stable subspace (let us point that this space depends on $A_{1}$ and $A_{2}$ but not $B_{0}$ or $B_{1}$ ) associated to the frequency $\zeta$ then we have:

$$
\text { ker } B_{0} \cap E^{s}(\zeta)=\{0\}
$$

which implies that the restriction of $B_{0}$ to $E^{s}(\zeta)$ is uniformly invertible (with respect to $\zeta$ ) (we refer to [Kre70-BGS07 or to Definition 3.3 for more details).

This inverse gives the coefficient of amplification/attenuation during the reflection against the side $\left\{x_{2}=0\right\}$ of a wave travelling from the interior to the boundary $\left\{x_{2}=0\right\}$ with frequency $\zeta$.

By localization arguments the uniform Kreiss-Lopatinskii condition on each side of the boundary is a necessary condition for the strong well-posedness of the strip problem.

However it is also known from the litterature (see for example [ST88- Cou05]) that when the uniform Kreiss-Lopatinskii condition breaks down on some specific parts of the frequency space (we refer to Section 3.1 for more details) then a new concept of well-posedness can be considered. More precisely such problems are referred as weakly well-posed in the sense that we still have existence and uniqueness of the solution but this solution is not as regular as (in Sobolev spaces) the source terms of the problem.

In the geometry of the half-space, these losses are well-understood. Indeed from the works of [ST88]Cou05- CG10]- Mar10]-Les07]- Ben14, the full characterization of the possible losses of derivatives is almost achieved (up to some really specific cases of study).

The strategy is the following: first show an energy estimate with a loss of derivatives and secondly saturate this estimate thanks to geometric optics expansions to show the sharpness of the estimate.

In the corner geometry this question has been investigated in Ben17 in which the author shows that the self-interaction phenomenon can be combined with the degeneracy of the uniform Kreiss-Lopatinskii condition to lead to a Hadamard instability formed by the accumulation of arbitrary many weak instabilities (that is to say losses of a finite number of derivatives).

In this article we address the same question but this time in the strip geometry. The main result of this paper is that in such a configuration the loss of derivatives increases with the maximal time of resolution. More precisely the number of losses is bounded by below by a step function of the form

$$
f(T)=\left[\frac{T}{\alpha}\right] \text {, where }[\cdot] \text { denotes the integer part function, }
$$

and where the coefficient $\alpha>0$ is explicit in terms of the group velocities of the hyperbolic operator (it is explicitly given by the matrices $A_{1}, A_{2}$ ) and corresponds to the minimal time needed to regenerate one

\footnotetext{
${ }^{1}$ It has been shown on some examples in $[\mathrm{Benb}$, but a rigorous proof has not been established yet
} 
phase by repeated reflections.

Consequently in terms of losses of derivatives the strip geometry can be seen as a median case between the half-space geometry (where the number of losses is finite whatever is the time scale) and the corner geometry (where this number can be made arbitrarily large independently of the time scale).

However let us remark that compared to the corner geometry for which the self-interaction phenomenon relies on some very restrictive operators $L(\partial)$ (more precisely on the geometry of its characteristic variety see [Ben17] form more details) in becomes generic in the strip geometry. As a consequence the repetition of a finite number of derivative is more generic for the strip problem than for the corner problem.

This kind of hyperbolic systems with increasing (with respect to time) losses of derivatives is not new. It can happen even if one deals with linear hyperbolic systems of partial differential equations defined in the full space.

Indeed, see CSFM14 and the many references therein, it occurs when the coefficients of the hyperbolic operator are of poor regularity or when the hyperbolicity assumption is weak as in [?].

Here one of the interesting point is that we recover the same kind of results but for hyperbolic operators having constant coefficients and under a rather strong hyperbolicity assumption. The growth of the loss comes from a repetition of weak instabilities which is possible because of the strip geometry and the particular choice of the boundary conditions. Moreover the lower bound on the number of losses is explicit in terms of the coefficients $A_{1}$ and $A_{2}$.

Another point of interest is that compared to Bena where the problem is almost the same (the only difference is the area of the frequency space where the uniform Kreiss-Lopatinskii condition breaks down), the behaviour of the solution differs totally. Indeed in Bena], this time dependence of the number of looses is absent and the solution behaves like the one in the half-space geometry.

The paper is organized as follow:

- in Section 2 we give a formal discussion explaining why this increasing with respect to time loss of derivatives is expected. This section is divided into a precise description in the framework of $2 \times 2$ systems and then a description of the needed generalizations to deal with the case where $N>2$.

- In Section 3 we introduce some notations, the assumptions and we state the main results of this article namely Theorem 3.2 dealing with the increasing with time loss of regularity and Theorem 3.3 the construction of geometric optics expansion for (1).

- Section 4 is devoted to the construction of geometric optics expansions, that is the proof of Theorem 3.3 It is the technical part of the article but the main ideas of the proof follows the ones described in Section 2 .

- At last Section 5 gives some examples and comments and/or possible extensions of the results.

\section{Formal discussion}

In this paragraph we discuss the main ideas in the proof of the main results.

Firstly we give a justification when the considered system has two equations. Consequently there is only two generated wave packets in the geometric optic expansion and the self-interaction phenomenon is quite simple.

Secondly we describe the needed generalizations to deal with the case $N>2$.

\subsection{Formal justification for $N=2$}

Consider the system (1) with $f \equiv 0$ and $g_{1} \equiv 0$. It is supposed to be of hyperbolic type so that it should satisfy the principle of finite speed of propagation. Consequently the non-zero source term on the boundary $\partial \Omega_{0}$ can not influence immediately the behaviour of the solution near the boundary $\partial \Omega_{1}$ (where it should be zero in absence of source term in the interior or on the boundary $\partial \Omega_{1}$ ). 
At least during a short time, there is no loss of generality by considering that the problem in the strip (1) acts like the problem in the half-space $\left\{x_{1} \in \mathbb{R}, x_{2}>0\right\}$.

Let $(\underline{\tau}, \underline{\eta}) \in \mathbb{R}^{2}$ be a fixed boundary frequency, it is natural to take as a first ansatz the one of the boundary value problem in the half-space $\left\{x_{1} \in \mathbb{R}, x_{2}>0\right\}$ :

$$
\begin{cases}L(\partial) u^{\varepsilon}=0 & \text { for } t \in]-\infty, T], x_{1} \in \mathbb{R}, x_{2}>0, \\ B_{0} u_{\mid x_{2}=0}^{\varepsilon}=\varepsilon e^{\frac{i}{\varepsilon}\left(t \underline{\tau}+x_{1} \underline{\eta}\right)} g_{0} & \text { for } t \in]-\infty, T], x_{1} \in \mathbb{R}, \\ u_{\mid t \leq 0}^{\varepsilon}=0 & \text { for } x_{1} \in \mathbb{R}, x_{2}>0,\end{cases}
$$

where $g_{0}$ is a smooth given amplitude vanishing for negative times.

The standard theory of linear geometric optics expansions see for example Rau12] applies and we shall consider for ansatz the function

$$
u^{\varepsilon}(t, x) \sim \sum_{n \geq 0} \varepsilon^{n}\left(e^{\frac{i}{\varepsilon}\left(t \underline{\tau}+x_{1} \underline{\underline{\eta}}+x_{2} \xi_{1}\right)} u_{n, 1}^{\varepsilon}(t, x)+e^{\frac{i}{\varepsilon}\left(t \underline{\tau}+x_{1} \underline{\underline{\eta}}+x_{2} \xi_{2}\right)} u_{n, 2}^{\varepsilon}(t, x)\right),
$$

where $\xi_{1}>0$ and $\xi_{2}<0$ are the (real) roots in the $\xi$-variable of the $\operatorname{dispersion}$ relation $\operatorname{det}\left(\underline{\tau} I+, \underline{\eta} A_{1}+\xi A_{2}\right)=$ 0 .

In the following the wave packet associated to $\xi_{1}>0$ is said to be incoming while the one associated to $\xi_{2}<0$ is refered as outgoing. Following, for example CG10, the outgoing phase can not influence the incoming one in the interior of the half-space. However at the level of the boundary of the half-space these waves packets can be mixed by the boundary matrix $B_{0}$. In the $W R$-configuration of [BGRSZ02] (see CG10-Cou05]) we are faced to a loss of derivatives. In the setting of geometric optics expansion this means that a source term on the boundary scaling like $O(\varepsilon)$ induces a geometric optics expansion of order $O(1)$. This explains why the scale in terms of $\varepsilon$ is not the same in (3) and in the ansatz.

To determine the leading order amplitudes, namely $u_{0,1}^{\varepsilon}$ and $u_{0,2}^{\varepsilon}$ and initialize the construction of the approximate solution, we inject the ansatz (4) in (3).

In particular when we consider the interior equation of (3) we obtain that $u_{0,1}^{\varepsilon}$ (resp. $u_{0,2}^{\varepsilon}$ ) lies in the so-called stable (resp. unstable) subspace of $L(\partial)$ (see (2)) and from Lax lemma (see [Lax57] and Lemma 4.1 its evolution in the interior is given by a transport equation (with constant coefficients that only depend on $L(\partial))$. Depending on the kind of the phase, these transport phenomena have a different behaviour. More precisely:

- the outgoing phase induces a transport phenomenon from the "right" to the "left". Consequently in (3) the only non trivial transportable information is the one in the interior that is zero. So this amplitude is, at least during a short time, zero and it is initially neglected in the WKB expansion.

- The incoming phase induces a transport phenomenon from the "left" to the "right". This time the non zero source term on the boundary $\partial \Omega_{0}$ can be transported. To solve this transport equation the trace of the amplitude on $\partial \Omega_{0}$ is required.

In the $W R$-class of [BGRSZ02, the degeneracy of the uniform Kreiss-Lopatinskii condition also induces a transport phenomenon along the boundary $\partial \Omega_{0}$, which turns on the transport phenomenon from the boundary to the interior of the domain.

More precisely, when plugging the ansatz (4) in the boundary condition (3) we obtain that

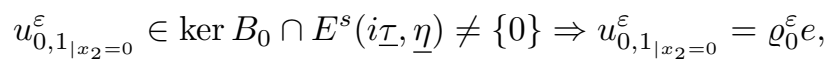

where $\varrho_{0}^{\varepsilon}$ is some (unknown) scalar function and $e$ is a generator $2^{2}$ of $\operatorname{ker} B_{0}$.

In [CG10] the determination of $\varrho_{0}^{\varepsilon}$ is made by a precise analysis of the boundary condition of order one. And the precise value of the transport operator along the boundary of the half-space acting on $\varrho_{0}^{\varepsilon}$ is given

\footnotetext{
${ }^{2}$ Because $N=2$ we have that $\operatorname{dim} \operatorname{ker} B_{0}=\operatorname{dim} E^{s}(i \underline{\tau}, \underline{\eta})=1$.
} 
in terms of the source term $g_{0}$. It permits to determine $u_{0,1}^{\varepsilon}$ by integration along the characteristics of the transport operator (in the interior).

However this transport phenomenon of the incoming phase, because it goes from the "left" to the right", will hit the boundary $\partial \Omega_{1}$ in finite time so that to have a global view of the problem in the strip we have to consider the influence of the reflection of this phase against this side of the boundary (see [Benb]). In order to do so we consider the initial boundary value problem in the half-space $\left\{x_{1} \in \mathbb{R}, x_{2}<1\right\}$ :

$$
\begin{cases}L(\partial) u^{\varepsilon}=0 & \text { for } t \in]-\infty, T], x_{1} \in \mathbb{R}, x_{2}<1 \\ B_{1} u_{\mid x_{2}=1}^{\varepsilon}=\widetilde{g}^{\varepsilon}, & \text { for } t \in]-\infty, T], x_{1} \in \mathbb{R} \\ u_{\mid t \leq 0}^{\varepsilon}=0 & \text { for } x_{1} \in \mathbb{R}, x_{2}<1\end{cases}
$$

where the non zero source term on the side $\partial \Omega_{1}$ comes from the impact of the incoming amplitude against this side.

As a consequence the initially neglected phase in the geometric optics expansion (namely the outgoing one for the side $\partial \Omega_{0}$ ) is now incoming for the side $\partial \Omega_{1}$. It can transport the boundary source term $\widetilde{g}^{\varepsilon}$ from $\partial \Omega_{1}$ to $\partial \Omega_{0}$. Because the boundary condition on $\partial \Omega_{1}$ is assumed to satisfy the uniform Kreiss-Lopatinskii condition, during this reflection there is no amplification phenomenon appearing in the geometric optic expansions.

However $u_{0,2}^{\varepsilon}$ the transported information will hit the side $\partial \Omega_{0}$ in finite time. And we shall investigate more precisely its reflection during this rebound.

When the amplitude associated to the outgoing phase impacts $\partial \Omega_{0}$ it is reflected in the first considered amplitude in the geometric optics expansion that is the incoming phase. Consequently this phase is regenerated back and it is precisely that we mean by self-interaction.

However recall that the boundary condition on $\partial \Omega_{0}$ is not assumed to satisfy the uniform KreissLopatinskii condition but only to be in the $W R$-class. As a consequence during this reflection a new amplification phenomenon/loss of derivative occurs.

Let $\alpha>0$ be the time needed to perform a regeneration of the incoming phase (we stress that it only depends on the hyperbolic operator) then we have the following possibilities:

- if $T<\alpha$ the leading order in the geometric optics expansion should be of order zero if one starts with a boundary source term of order one compared to $\varepsilon$. Because the time is not large enough to see the self-interaction. In particular, the results of [CG10] hold.

- if $\alpha \leq T<2 \alpha$ then the final time of resolution is large enough to see the reflection. The incoming amplitude is regenerated and during this reflection we lose a second derivative. For such final times the considered ansatz is of order zero for a boundary source term of order $\varepsilon^{2}$. However in (4) the term $u_{0,1}^{\varepsilon}$ (that is the one carrying the two amplifications/losses) should be zero under the threshold $t<\alpha$.

We can reiterate exactly the same arguments for times on the form $n \alpha \leq T<(n+1) \alpha$ showing that on these time scales the phase responsible of the degeneracy of the uniform Kreiss-Lopatinskii condition has been regenerated exactly $n$-times so that if one starts with a source term on the boundary of order $\varepsilon^{n+1}$ then the leading order in the WKB expansion should be of order zero (the +1 comes from the fact that for $W R$ problems the first loss of derivatives is immediate) if the boundary source term is non zero.

Moreover in (4) the term carrying the $n+1$ amplifications, namely $u_{0,1}^{\varepsilon}$ is zero under the threshold $t<n \alpha$. Indeed because of the definition of $\alpha$ and the fact $n \alpha \leq T<(n+1) \alpha$, this term comes from the source term $g_{0}$ which has been regenerated $n$ times.

This remark is not harmless at all because we have to keep in mind that in the analysis of [CG10] the boundary condition of order one have to be considered to obtain the value of $\varrho_{0}^{\varepsilon}$ so that the term carrying $n+1$ amplifications in the WKB expansion should a priori depend on the term of order one carrying $n$ amplifications. This is in fact impossible if we fix $n$ such that $n \alpha \leq T<(n+1) \alpha$ because the term carrying 
$n$ amplifications in the WKB expansion, namely $u_{1,1}^{\varepsilon}$ can not be regenerated by self-interaction.

As a consequence we obtain the increasing in time loss of derivatives claimed in Theorem 3.2 because of the construction of the WKB expansion.

\subsection{Extension to the framework $N>2$}

The method described in the case $N=2$ in the previous paragraph can be extended to systems with more than two equations. However this extension implies some important technical difficulties that are listed below:

- generically the source term on the boundary $\partial \Omega_{0}$ induces more than one incoming phase. Let $N_{\mathscr{I}}$ be the number of incoming phases (that is to say the number of positive real roots in the $\xi$-variable of the dispersion relation $\left.\operatorname{det}\left(\underline{\tau} I+\underline{\eta} A_{1}+\xi A_{2}\right)=0\right)$.

Then each of the linear phases $\varphi_{k}:=\underline{\tau} t+\eta x_{1}+\xi_{k} x_{2}$ travels to the boundary $\partial \Omega_{1}$ and reaches it after the time of travel $\alpha_{k}>0$. During each reflection against the side $\partial \Omega_{1}$ there is generically more than one generated outgoing phase. Let $N_{\mathscr{O}}$ be the number of outgoing phases (the number of negative roots of the dispersion relation). Because we have in mind a constantly hyperbolic operator (see Assumption 3.1), we have $N_{\mathscr{I}}+N_{\mathscr{O}} \leq N$.

Like in the case $N=2$, each of these outgoing phases $\varphi_{\ell}$ goes back to the boundary $\partial \Omega_{0}$ and impacts it after the time of travel $\alpha_{\ell}>0$. During each reflection all the incoming phases are regenerated and we observe a loss of a second derivative and/or an amplification at the level of the WKB expansion. Thus the self-interaction phenomenon is more elaborate than in the case $N=2$ because there is $N_{\mathscr{O}}$ paths of phases regenerated for a fixed incoming phase. However this more complicated self-interaction phenomenon is generic in strip problems and was already considered in [Benb].

What may seem annoying in the previous arguments is that each of these paths induces a different time of regeneration. However the discussion exposed in the framework $N=2$ can be extended to the case $N>2$ but the time of appearance of the losses of derivatives is now given by $\alpha:=\min _{k, \ell} \alpha_{k}+\alpha_{\ell}$ that is to say the minimal time of travel in the regeneration process. Indeed the main term in the WKB expansion that is the one carrying the maximal number of losses is the one associated to the wave packet with the higher velocity.

- Another point to be considered when $N>2$ is that even if we still assume that the boundary matrix $B_{0}$ gives a problem in the $W R$-class so that essentially $\operatorname{dim}\left(\operatorname{ker} B_{0} \cap E^{s}(i \underline{\tau}, \underline{\eta})\right)=1$, this does not generically implies that $e$ defined in (2) is a generator of $\operatorname{ker} B_{0}$ because, $\operatorname{dim} E^{s}(\overline{i \underline{\tau}}, \underline{\eta})=\operatorname{dim} \operatorname{ker} B_{0}=1$ does not hold any more.

However, we can use the analysis of [CG10] to write a priori

$$
u_{0,1_{\mid x_{2}=0}^{\varepsilon}}^{\varepsilon}=\varrho_{0}^{\varepsilon} e+\nu_{0}^{\varepsilon} v_{0, *},
$$

instead of (5), where $\varrho_{0}^{\varepsilon}$ and $\nu_{0}^{\varepsilon}$ are scalar (unknowns) functions, $e$ and $v_{0, *}$ are defined in such a way (see Definition 4.2 for more details) that the amplification during a reflection against $\left\{x_{2}=0\right\}$ is carried on $e$ but not on $v_{0, *}$. In other words the two terms in (6) (that can a priori interact together in the boundary condition) appear at different time scales, because they are not associated to the same loss of regularity.

A precise analysis of the self-interaction phenomenon then shows that $\nu_{0}^{\varepsilon}$ is explicitly given by the self-interaction operator (see [Benb]) applied to some function of $\varrho_{0}^{\varepsilon} e$ evaluated one time $t-\alpha>0$ so that this term is in fact zero by the (new) definition of $\alpha$.

Consequently we can conclude, up to several reformulations of the equations, that the $u_{0, k}^{\varepsilon}$ (the amplitudes associated to the incoming phases) are zero under the threshold $t<n \alpha$ and depend explicitly on $g_{0}$ which has been regenerated $n$ times, exactly like in the framework $N=2$. 
The ideas exposed in this section are illustrated in the following figures.

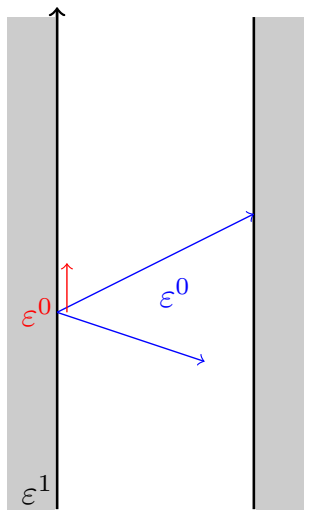

Figure 1: Appearance of the characteristics induced by the source term $N=3$ and $p=2$ and first amplification.

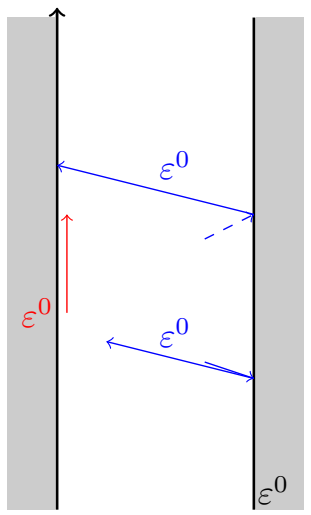

Figure 2: Appearance of the characteristics induced after the first reflection 


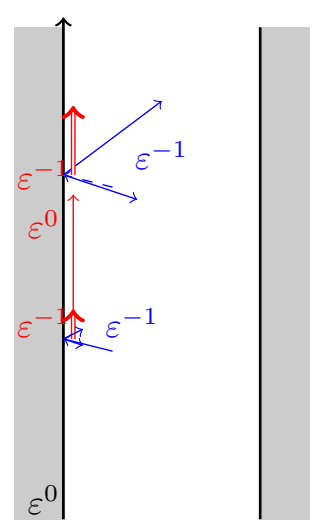

Figure 3: Appearance of the characteristics after the second reflection, second amplification

\section{$3 \quad$ Notations and main results}

In this article $T>0$ stands for the final time of resolution of the strip problem (1).

For convenience we define:

$$
\begin{aligned}
\Omega & :=\mathbb{R} \times] 0,1\left[, \partial \Omega_{0}:=\mathbb{R} \times\{0\} \simeq \mathbb{R} \times\{1\}=: \partial \Omega_{1},\right. \\
\Omega_{T} & \left.\left.\left.\left.:=]-\infty, T] \times \Omega, \partial \Omega_{0, T}:=\right]-\infty, T\right] \times \partial \Omega_{0} \simeq\right]-\infty, T\right] \times \partial \Omega_{1}=: \partial \Omega_{1, T} .
\end{aligned}
$$

We consider the following frequency space obtained after Laplace transform in time $t \leadsto \rightsquigarrow \quad \sigma:=\gamma+i \tau$, with $\gamma>0$ and $\tau \in \mathbb{R}$, and Fourier transform in the $x_{1}$ variable $x_{1} \leadsto \eta \in \mathbb{R}$;

$$
\Xi:=\{\zeta:=(\sigma=\gamma+i \tau, \eta) \in \mathbb{C} \times \mathbb{R} \backslash \Omega \geq 0\}, \Xi_{0}:=\Xi \cap\{\Omega=0\} .
$$

Finally for $a, b \in \mathbb{C}^{p}$ we denote the scalar product on $\mathbb{C}^{p}$ by $a \cdot b$.

\subsection{Notations and assumptions}

\subsubsection{About the operator $L(\partial)$}

Firstly we assume that the operator $L(\partial)$ is hyperbolic in the following sense:

Assumption 3.1 [Constant hyperbolicity] The system (1) is constantly hyperbolic that is there exist $1 \leq$ $M \leq N$, real valued analytic functions $\lambda_{1}, \ldots, \lambda_{M}$ on $\mathbb{R}^{2} \backslash\{0\}$ and positive integers $\mu_{1}, \ldots, \mu_{M}$ such that:

$$
\forall \xi=\left(\xi_{1}, \xi_{2}\right) \in \mathbb{S}, \operatorname{det}\left(\tau I+\xi_{1} A_{1}+\xi_{2} A_{2}\right)=\prod_{j=1}^{M}\left(\tau+\lambda_{j}(\xi)\right)^{\mu_{j}},
$$

with $\lambda_{1}(\xi)<\cdots<\lambda_{M}(\xi)$ and the eigenvalues $\lambda_{j}(\xi)$ of $\xi_{1} A_{1}+\xi_{2} A_{2}$ are semi-simple.

The following assumption is also rather classical in the study of hyperbolic boundary value problems and it is satisfied by many examples of physical interest (for example the wave equation or the linearisation of Euler equations see Paragraph 5.1P

Assumption 3.2 [Non characteristic boundary condition] We assume that $A_{2}$ is non singular, meaning that $\operatorname{det} A_{2} \neq 0$. Moreover the boundary matrices $B_{0}$ and $B_{1}$ induce the good number of boundary conditions that is $B_{0} \in \mathbf{M}_{p, N}(\mathbb{R})$ and $B_{1} \in \mathbf{M}_{N-p, N}(\mathbb{R})$ where $p$ stands for the number of positive real roots of $A_{2}$.

Thanks to Assumption 3.2 we can perform a Laplace transform with respect to time $(t \leadsto \sigma)$ and a Fourier transform $\left(x_{1} \leadsto \eta\right)$ in the $x_{1}$-variable in (1) to write it under its so-called resolvent form. 
Let $\zeta \in \Xi \backslash \Xi_{0}$, then $(1)$ becomes

$$
\left\{\begin{array}{l}
\left.\frac{\mathrm{d}}{\mathrm{d} x_{d}} \widehat{u}(\zeta)=\mathscr{A}(\zeta) \widehat{u}(\zeta)+\widehat{f}(\zeta) \quad \text { for } x_{d} \in\right] 0,1[ \\
B_{0} \widehat{u}_{x_{2}=0}(\zeta)=\widehat{g}_{0}(\zeta) \\
B_{1} \widehat{u}_{\mid x_{2}=1}(\zeta)=\widehat{g}_{1}(\zeta)
\end{array}\right.
$$

in which the resolvent matrix $\mathscr{A}(\zeta)$ is defined by $\mathscr{A}(\zeta):=-A_{2}^{-1}\left(\sigma I+i \eta A_{1}\right)$ where $\widehat{\cdot}$ stands for the FourierLaplace transform.

The following lemma due to Hersh in Her63. ensures that as long as the frequency parameter $\sigma$ admits a non vanishing real part then the same holds for the eigenvalues of $\mathscr{A}(\zeta)$. Consequently the evolution of the solution of (7) is ruled by generalized eigenmodes associated to generalized eigenvalues with strictly positive (resp. strictly negative) real parts which are responsible of exponential increasing (resp. decreasing) components of the solution with respect to the $x_{2}$-variable.

Lemma 3.1 (Hersh [Her63]) Under Assumptions 3.1 and 3.2. let $\zeta \in \Xi \backslash \Xi_{0}$ then the eigenvalues of $\mathscr{A}(\zeta)$ have non vanishing real part.

We denote by $E^{s}(\zeta)$ (resp. $E^{u}(\zeta)$ ) the generalized eigenspace generated by the eigenmodes associated to negative (resp. positive) real part.

For all $\zeta \in \Xi \backslash \Xi_{0}$, dim $E^{s}(\zeta)=p$ and $\operatorname{dim} E^{u}(\zeta)=N-p$ and we have $\mathbb{C}^{N}=E^{s}(\zeta) \oplus E^{u}(\zeta)$.

However, Kreiss's half-space theory [Kre70] requires to have a look to the boundary of the frequency space, namely $\Xi_{0}$. For such frequencies it is clear that Hersh lemma generalically does not hold anymore because of the possible degeneracy of the real part of the eigenvalues. To overcome this "difficulty" we use a more precise result due to [Kre70] for strictly hyperbolic systems and then generalized by [Mét00] for constantly hyperbolic systems. It is the so-called block structure theorem.

Theorem 3.1 (Block structure) Under Assumptions 3.1 and 3.2, for all $\zeta \in \Xi$, there exist a neighbourhood $\mathscr{V}$ of $\underline{\zeta}$ in $\Xi$, an integer $L \geq 1$, a partition $N=\mu_{1}+\cdots+\mu_{L}$, with $\mu_{1}, \ldots, \mu_{L} \geq 1$ and an invertible matrix $T$, regular on $\mathscr{V}$ such that:

$$
\forall \zeta \in \mathscr{V}, T^{-1}(\zeta) \mathscr{A}(\zeta) T(\zeta)=\operatorname{diag}\left(\mathscr{A}_{1}(\zeta), \cdots, \mathscr{A}_{L}(\zeta)\right)
$$

where the blocks $\mathscr{A}_{j}(\zeta) \in \mathbf{M}_{\mu_{j} \times \mu_{j}}(\mathbb{C})$ satisfy one of the following alternatives:

i) all the elements in the spectrum of $\mathscr{A}_{j}(\zeta)$ have positive real part.

ii) All the elements in the spectrum of $\mathscr{A}_{j}(\zeta)$ have negative real part.

iii) $\mu_{j}=1, \mathscr{A}_{j}(\underline{\zeta}) \in i \mathbb{R}, \partial_{\omega} \mathscr{A}_{j}(\underline{\zeta}) \in \mathbb{R} \backslash\{0\}$ and $\mathscr{A}_{j}(\zeta) \in i \mathbb{R}$ for all $\zeta \in \Xi_{0} \cap \mathscr{V}$.

iv) $\mu_{j}>1$ and there exists $k_{j} \in i \mathbb{R}$ such that

$$
\mathscr{A}_{j}(\underline{\zeta})=\left[\begin{array}{ccc}
k_{j} & i & 0 \\
& \ddots & i \\
0 & & k_{j}
\end{array}\right],
$$

where the coefficient in the lower left corner of $\partial_{\omega} \mathscr{A}_{j}(\underline{\zeta}) \in \mathbb{R} \backslash\{0\}$ and for all $\zeta \in \Xi_{0} \cap \mathscr{V}, \mathscr{A}_{j}(\zeta) \in i \mathbf{M}_{\mu_{j} \times \mu_{j}}(\mathbb{R})$.

Theorem 3.1 immediately implies that $\Xi_{0}$ can be decomposed in the following way:

Definition 3.1 We have the partition

$$
\Xi_{0}=\mathbb{E} \cup \mathbb{M} \cup \mathbb{H} \cup \mathbb{G}
$$

where we defined:

- $\mathbb{E}$ the elliptic area. That is the set of frequencies $\zeta \in \Xi_{0}$ such that Theorem 3.1 is satisfied with blocks of type $i$ ) and ii) only. 
- $\mathbb{M}$ the mixed area. That is the set of frequencies $\zeta \in \Xi_{0}$ such that Theorem 3.1 is satisfied with blocks of type i), ii) and one of type iii).

- $\mathbb{H}$ the hyperbolic area. That is the set of frequencies $\zeta \in \Xi_{0}$ such that Theorem 3.1 is satisfied with blocks of type iii) only.

- $\mathbb{G}$ the glancing area. That is the set of frequencies $\zeta \in \Xi_{0}$ such that Theorem 3.1 is satisfied with one block of type iv).

As already mentioned in the introduction we are here interested in the construction of geometric optics expansions associated to hyperbolic frequencies, that is $\mathbb{H}$.

When $\zeta \in \mathbb{H}$ then the stable and unstable subspaces can be easily described; this precise description of $E^{s}(\zeta)$ and $E^{u}(\zeta)$ permits to define the group velocities which play a crucial role in the construction. Indeed in a classical way we have:

Definition 3.2 (Group velocities) Let $\zeta \in \mathbb{H}$ then we have

$$
\mathbb{C}^{N}=E^{s}(\zeta) \oplus E^{u}(\zeta)=\left(\bigoplus_{k \in \mathscr{I}} \operatorname{ker} \mathscr{L}\left(i \tau, \eta, \xi_{k}\right)\right) \oplus\left(\bigoplus_{\ell \in \mathscr{O}} \operatorname{ker} \mathscr{L}\left(i \tau, \eta, \xi_{\ell}\right)\right),
$$

where $\mathscr{L}\left(\xi_{0}, \xi_{1}, \xi_{2}\right):=\xi_{0} I+\xi_{1} A_{1}+\xi_{2} A_{2}$ is the symbol of $L(\partial)$ and where $\mathscr{I}$ (resp. $\mathscr{O}$ ) is the incoming (resp. outgoing) set defined by the set of $m$ such that $\partial_{\xi} \lambda_{k_{m}}\left(\eta, \xi_{m}\right)>0\left(\right.$ resp. $\partial_{\xi} \lambda_{k_{m}}\left(\eta, \xi_{m}\right)<0$ ) (see for example [CG10]).

For $k \in \mathscr{I} \cup \mathscr{O}$ we introduce the group velocity associated to $k$ by defining $\mathbf{v}_{k}:=\nabla \lambda_{m_{k}}\left(\eta, \xi_{k}\right)$.

\subsubsection{About the boundary conditions}

In this paragraph we firstly recall the condition ensuring the strong well-posedness of the hyperbolic boundary value problem in the half-space, namely the uniform Kreiss-Lopatinskii condition, and then we give another type of boundary condition for which the problem in the half-space in known to be weakly well-posed, namely the $W R$ boundary conditions of [BGRSZ02].

Definition 3.3 [Uniform Kreiss-Lopatinskii condition] The strip hyperbolic problem (1) satisfies the uniform Kreiss-Lopatinskii condition on the side $\partial \Omega_{0}$ (resp. $\left.\partial \Omega_{1}\right)$ if for all $\zeta \in \Xi$ we have:

$$
\text { ker } \left.B_{0} \cap E^{s}(\zeta)=\{0\} \quad \text { resp. } \operatorname{ker} B_{1} \cap E^{u}(\zeta)=\{0\}\right) .
$$

In such a situation the restriction of the matrix $B_{0}$ (resp. $\left.B_{1}\right)$ to the stable (resp. unstable) subspace $E^{s}(\zeta)$ (resp. $\left.E^{u}(\zeta)\right)$ is invertible and we denote

$$
\phi_{0}=\phi_{0}(\zeta):=B_{0_{\mid E^{s}(\zeta)}^{-1}}^{-r e s p .} \phi_{1}=\phi_{1}(\zeta):=B_{1_{\mid E^{u}(\zeta)}^{-1}}^{-1} .
$$

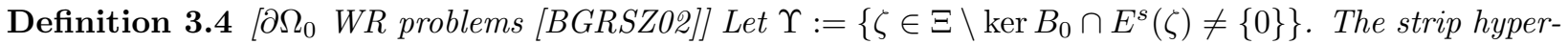
bolic problem (1) is said to be in the WR-class for the side $\partial \Omega_{0}$ if it satisfies the following conditions:

- the strip problem (1) satisfies the (weak) Kreiss-Lopatinskii condition that is $\Upsilon \cap\left(\Xi \backslash \Xi_{0}\right)=\emptyset$.

- $\Upsilon \neq \emptyset$ and $\Upsilon \subset \mathbb{H}$.

- For all $\underline{\zeta} \in \Upsilon$, there exist a neighbourhood $\mathscr{V}$ of $\underline{\zeta}$ in $\Xi$, a regular basis $\left(e_{1}, e_{2}, \ldots, e_{p}\right)(\zeta)$ of $E^{s}(\zeta)$ on $\mathscr{V}$, an invertible and smooth matrix $P(\zeta)$ on $\mathscr{V}$ and a smooth real valued function $\Theta$ on $\mathscr{V}$ such that the following holds

$$
\forall \zeta \in \mathscr{V}, B_{0}\left[e_{1}, e_{2}, \ldots, e_{p}\right](\zeta)=P^{-1}(\zeta) \operatorname{diag}(\gamma+i \Theta(\zeta), 1, \ldots, 1) P(\zeta) .
$$

Crudely speaking $W R$ boundary conditions are such that the uniform Kreiss-Lopatinskii condition degenerates for at least one boundary frequency $\underline{\zeta}$ of hyperbolic type (meaning that the resolvent matrix $\mathscr{A}(\underline{\zeta})$ is diagonalizable with purely imaginary eigenvalues) and in such a way that $\operatorname{dim}\left(\operatorname{ker} B_{0} \cap E^{s}(\underline{\zeta})\right)=1$. Let 
us stress that such boundary conditions are generic (see the examples in BGRSZ02 and Figures 4.5).

For such a boundary condition we know from [Cou05] and CG10] that the problem in the half-space is weakly well-posed in the sense that it admits a unique solution $u$ and that this solution satisfies the energy estimate: there exists $C>0$ such that for all $\gamma$ large enough

$$
\gamma\|u\|_{L_{\gamma}^{2}\left(\widetilde{\Omega}_{T}\right)}^{2}+\left\|u_{\mid x_{d}=0}\right\|_{L_{\gamma}^{2}\left(\partial \widetilde{\Omega}_{T}\right)}^{2} \leq C\left(\frac{1}{\gamma}\|f\|_{L_{\gamma}^{2}\left(\widetilde{\Omega}_{T}\right)}^{2}+\frac{1}{\gamma^{3}}\left\|\nabla_{t, x^{\prime}} f\right\|_{L_{\gamma}^{2}\left(\widetilde{\Omega}_{T}\right)}^{2}+\left\|g_{0}\right\|_{L_{\gamma}^{2}\left(\widetilde{\partial \Omega}_{T}\right)}^{2}+\frac{1}{\gamma^{2}}\left\|\nabla g_{0}\right\|_{L_{\gamma}^{2}\left(\partial \widetilde{\Omega}_{T}\right)}^{2}\right)
$$

where $\widetilde{\Omega}_{T}$ stands for the half space $\left.\left.\left\{(t, x)=\left(t, x^{\prime}, x_{d}\right) \in\right]-\infty, T\right] \times \mathbb{R}^{d-1} \times\right] 0, \infty[\}$ and where $\|\cdot\|_{L_{\gamma}^{2}(X)}$ is the time weighted norm defined by $\|\cdot\|_{L_{\gamma}^{2}(X)}:=\left\|e^{-\gamma t} \cdot\right\|_{L^{2}(X)}$, where $X \subset \widetilde{\Omega}_{T}$. So for such a degeneracy of the uniform Kreiss-Lopatinskii condition the solution exhibits a loss of one derivative in the interior and a loss of one derivative on the boundary.

In this article we are interested in the influence of one boundary condition which does not satisfies the uniform Kreiss-Lopatinskii condition but which is only in the $W R$-class on the behaviour of the solution of the strip problem ${ }^{3}$

Assumption 3.3 The boundary conditions of the strip hyperbolic problem (1) satisfy the following:

- the boundary condition on $\partial \Omega_{0}$ is in the WR-class in the sense of Definition 3.4.

- The boundary condition on $\partial \Omega_{1}$ satisfies the uniform Kreiss-Lopatinskii condition in the sense of Definition 3.3 .

\subsection{Main result and proof}

The main result of this paper states that if the solution of (1) exists then it has to develop a time depending number of losses of derivatives. More precisely Theorem 3.2 gives an explicit lower bound on the number of losses of derivatives. This lower bound is a step function depending on $T$ and of the group velocities of the problem. In particular such a problem can not be (weakly) well-posed in infinity time.

\subsubsection{Statement}

Let $T>0$ and consider the strip problem 4

$$
\begin{cases}\partial_{t} u+A_{1} \partial_{1} u+A_{2} \partial_{2} u=f & \text { on } \Omega_{T}, \\ B_{0} u_{\mid x_{2}=0}=g_{0} & \text { on } \partial \Omega_{0, T}, \\ B_{1} u_{\mid x_{2}=1}=0 & \text { on } \partial \Omega_{1, T}, \\ u_{\mid t \leq 0}=0 & \text { on } \Omega .\end{cases}
$$

Then the main result is the following:

Theorem 3.2 Let $K>0$ be given; under Assumptions 3.1, 3.2 and 3.3, assume that the strip problem (10) is weakly well-posed in the sense that for all $f \in L^{2}\left(\Omega_{T}\right)$ and $g_{0} \in H^{K}\left(\partial \Omega_{0, T}\right)$ vanishing for negative times, the solution $u$ exists and satisfies the following energy estimate: there exists $C_{T}>0$ such that

$$
\|u\|_{L^{2}\left(\Omega_{T}\right)}^{2} \leq C_{T}\left(\|f\|_{L^{2}\left(\Omega_{T}\right)}^{2}+\left\|g_{0}\right\|_{H^{K}\left(\partial \Omega_{0, T}\right)}^{2}\right),
$$

then $K \geq\left[\frac{T}{\alpha}\right]+1$ where $\alpha>0$ is the minimal time of regeneration given by

$$
\alpha:=\min _{(k, \ell) \in \mathscr{I} \times \mathscr{O}}\left\{\frac{1}{\mathbf{v}_{k, 2}}-\frac{1}{\mathbf{v}_{\ell, 2}}\right\}>0,
$$

where $\mathbf{v}_{\cdot, 2}$ stands for the second component of the group velocity introduced in Definition 3.2.

\footnotetext{
${ }^{3}$ The results of this paper hold mutatis mutandis if both boundary conditions are in the $W R$-class but we choose, to simplify the computations in the proofs, to consider only one boundary condition in the $W R$-class. By convention let us say that the boundary condition on $\partial \Omega_{0}$ is in the $W R$-class.

${ }^{4}$ Because of the linearity of 1 , the case where $g_{1} \not \equiv 0$ is of little interest in its own. A careful study however shows that Theorem 3.2 holds if one changes the subscripts 0 into 1 up to modify a little the lower bound on $K$.
} 
The proof of Theorem 3.2 relies on the construction of the geometric optics expansion associated to 10 . By linearity of 10 and to simplify as much as possible the construction, we assume that $f \equiv 0$.

Consequently, consider the highly oscillating boundary value problem in the strip $\Omega_{T}$ : for $0<\varepsilon \ll 1$,

$$
\begin{cases}\partial_{t} u^{\varepsilon}+A_{1} \partial_{1} u^{\varepsilon}+A_{2} \partial_{2} u^{\varepsilon}=0 & \text { on } \Omega_{T} \\ B_{0} u_{\mid x_{2}=0}^{\varepsilon}=\varepsilon^{\left[\frac{T}{\alpha}\right]+1} g_{0}^{\varepsilon} & \text { on } \partial \Omega_{0, T}, \\ B_{1} u_{\mid x_{2}=1}^{\varepsilon}=0 & \text { on } \partial \Omega_{1, T} \\ u_{\mid t \leq 0}^{\varepsilon}=0 & \text { on } \Omega\end{cases}
$$

where the source term on the boundary $\partial \Omega_{0, T}$ is given by

$$
g_{0}^{\varepsilon}\left(t, x_{1}\right):=e^{\frac{i}{\varepsilon}\left(\underline{\tau} t+\underline{\eta} x_{1}\right)} g_{0}\left(t, x_{1}\right),
$$

for some fixed frequency $(\underline{\tau}, \underline{\eta}) \in \mathbb{R}^{2}$ and some given amplitude $g_{0} \in H^{\infty}\left(\partial \Omega_{0, T}\right)$ vanishing for negative times.

The theorem giving the geometric optics expansion is the following

Theorem 3.3 (Geometric optics expansion for (13)) Under Assumptions 3.1, 3.2 and 3.3 also assume

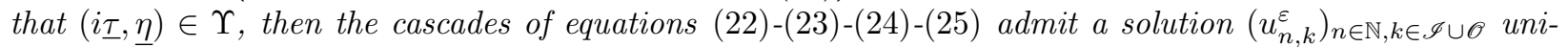
formly bounded with respect to $\varepsilon$ where for all $k \in \mathscr{I} \cup \mathscr{O}$ and for all $n \in \mathbb{N}, u_{n, k}^{\varepsilon} \in \mathscr{P}_{n}$ (the profil space defined in (21)). Moreover there exists $g_{0}$ in (14) such that the $u_{0, k}^{\varepsilon}$ are not identically zero.

To conclude this section we give a proof of Theorem 3.2 under the hypothesis that Theorem 3.3 holds. The proof of Theorem 3.3 that is the construction of the WKB expansion is the technical part of the article and is performed in Section 4 .

\subsubsection{Proof of Theorem 3.2 using Theorem 3.3}

The proof exposed follows the one of [CG10] (see also [Ben17]). We proceed by contradiction. Assume that $K<\left[\frac{T}{\alpha}\right]+1$ and consider $u^{\varepsilon}$ the exact solution of $\left[13\right.$. Define the approximate solution $u_{a p p}^{\varepsilon}$ given by the truncated WKB expansion. More precisely

$$
u_{a p p}^{\varepsilon}:=\sum_{k \in \mathscr{I} \cup \mathscr{O}} e^{\frac{i}{\varepsilon} \varphi_{k}} u_{0, k}^{\varepsilon}+\varepsilon \sum_{k \in \mathscr{I} \cup \mathscr{O}} e^{\frac{i}{\varepsilon} \varphi_{k}} u_{1, k}^{\varepsilon}
$$

where the $u_{n, k}^{\varepsilon}$ are the one given by Theorem 3.3. By construction (see the cascades of equations 222-23)(24)-25) the remainder $u^{\varepsilon}-u_{a p p}^{\varepsilon}$ satisfies the strip problem:

$$
\begin{cases}L(\partial)\left(u^{\varepsilon}-u_{a p p}^{\varepsilon}\right)=f^{\varepsilon} & \text { on } \Omega_{T}, \\ B_{0}\left(u^{\varepsilon}-u_{a p p}^{\varepsilon}\right)_{\mid x_{2}=0}=0 & \text { on } \partial \Omega_{0, T}, \\ B_{1}\left(u^{\varepsilon}-u_{a p p}^{\varepsilon}\right)_{\mid x_{2}=1}=0 & \text { on } \partial \Omega_{1, T} \\ \left(u^{\varepsilon}-u_{a p p}^{\varepsilon}\right)_{\mid t \leq 0}=0 & \text { on } \Gamma .\end{cases}
$$

where

$$
f^{\varepsilon}:=\varepsilon \sum_{k \in \mathscr{I} \cup \mathscr{O}} e^{\frac{i}{\varepsilon} \varphi_{k}} L(\partial) u_{1, k}^{\varepsilon} .
$$

From the energy estimate (11) applied to (13) and 10 respectively we obtain

$$
\left\|u^{\varepsilon}-u_{a p p}^{\varepsilon}\right\|_{L^{2}\left(\Omega_{T}\right)} \leq C_{T}\left\|f^{\varepsilon}\right\|_{L^{2}\left(\Omega_{T}\right)} \sim \varepsilon \text { and }\left\|u^{\varepsilon}\right\|_{L^{2}\left(\Omega_{T}\right)} \leq C_{T}\left\|g_{0}^{\varepsilon}\right\|_{L^{2}\left(\partial \Omega_{0, T}\right)} \sim \varepsilon^{\left[\frac{T}{\alpha}\right]+1-K} .
$$

From the triangle inequality and using the fact $K<\left[\frac{T}{\alpha}\right]+1, u_{a p p}^{\varepsilon}$ is $O\left(\varepsilon^{\lambda}\right)$ with $\lambda>0$. This contradicts the fact that the $u_{0, k}^{\varepsilon}$ are not identically zero. 


\section{Construction of the geometric optics expansion}

\subsection{Useful notations for geometric optics expansions}

This paragraph lists several notations that will be used in the following in order to simplify the reading.

Firstly let us point that the index $\ell$ will always be associated to outgoing modes and that the index $k$ will refer to incoming modes (or exceptionally to incoming and outgoing modes (in order to save some notations)) (we refer to Definition 3.2). We recall that the set of incoming phases is denoted by $\mathscr{I}$, the set of outgoing phases is denoted by $\mathscr{O}$ and finally we use the short hand notation $\mathscr{H}:=\mathscr{I} \cup \mathscr{O}$.

The operator $\left(I-\mathcal{S}^{\varepsilon}\right)$ (see (36) for a precise definition) denotes the self-interaction operator. The transport operator along the boundary $\partial \Omega_{0}$ (which naturally appears for $W R$ boundary conditions see CG10]) is denoted by $\mathcal{T}$ (see (41) for a precise definition).

As it is common in the construction of geometric optics expansions we use the following projections:

Definition 4.1 (Projections) Consider $\underline{\zeta}=(i \underline{\tau}, \underline{\eta}) \in \mathbb{H}$, let for $k=1, \ldots, \# \mathscr{H}, f_{k}:=\left(i \underline{\tau}, \underline{\eta}, \xi_{k}\right)$ where $\xi_{k}$ stands for the $k^{i t h}$ (real) root in the $\xi$ variable of $\operatorname{det} \mathscr{L}(i \underline{\tau}, \underline{\eta}, \xi)=0$. We define the following projections:

- $P^{k}$ the projection on $\operatorname{ker} \mathscr{L}\left(i \underline{\tau}, \underline{\tau}, \xi_{k}\right)$ with respect to the direct sum (8).

- $Q^{k}$ the projection on $A_{2} \operatorname{ker} \mathscr{L}\left(i \underline{\tau}, \underline{\tau}, \xi_{k}\right)$ with respect to the direct sum $!^{5}\left(A_{2}(8)\right)$.

- Finally we define $R^{k}$ the pseudo-inverse of $\mathscr{L}\left(i \underline{\tau}, \underline{\eta}, \xi_{k}\right)$ characterized in a unique way by the relations:

$$
R^{k} \mathscr{L}\left(i \underline{\tau}, \underline{\eta}, \xi_{k}\right)=I-P^{k} \text { and } P^{k} R^{k}=R^{k} Q^{k}=0 .
$$

At last we define here some elements that are used when one deals with geometric optic expansions in the $W R$ class, see CG10.

Definition 4.2 Under Assumption 3.3 and let $\underline{\zeta} \in \Upsilon$ we introduce:

- a vector $e \in \mathbb{R}^{N} \backslash\{0\}$ such that $\operatorname{ker} B_{0} \cap E^{s}(\underline{\zeta})=\operatorname{span}\{e\}$. And for convenience for all $k \in \mathscr{I}$, we define $e_{k}:=P^{k}$.

- $A$ vector $b \in \mathbb{R}^{p} \backslash\{0\}$ such that we have $B_{0} E^{s}(\underline{\zeta})=\left\{v \in \mathbb{C}^{p} \backslash b \cdot v=0\right\}$.

- A vector space $E_{*}^{s}(\zeta)$ which is a supplementary vector space to span $\{e\}$ in $E^{s}(\underline{\zeta})$. Moreover the restriction of $B_{0}$ to $E_{*}^{s}(\underline{\zeta})$ is invertible and we denote its inverse by $\phi_{0, *}:=\phi_{0, *}(\underline{\zeta})=B_{0_{\mid E_{*}^{s}(\zeta)}^{-1}}^{-1}$.

\subsection{The cascades of equations}

In the general framework we consider the highly oscillating system of equations for $T>0$

$$
\begin{cases}L(\partial) u^{\varepsilon}=0 & \text { in } \Omega_{T}, \\ B_{0} u_{\mid x_{2}=0}^{\varepsilon}=\varepsilon^{K+1} g^{\varepsilon} & \text { on } \partial \Omega_{0, T}, \\ B_{1} u_{\mid x_{2}=1}^{\varepsilon}=0 & \text { on } \partial \Omega_{1, T}, \\ u_{\mid t \leq 0}^{\varepsilon}=0 & \text { on } \Omega,\end{cases}
$$

where $K:=K(T)=\left[\frac{T}{\alpha}\right]$ (where we recall that $[\cdot]$ denotes the integer part function) with $\alpha:=\min _{k \in \mathscr{I}, \ell \in \mathscr{O}} \frac{1}{\mathbf{v}_{k, 2}}-$ $\frac{1}{\mathbf{v}_{\ell, 2}}$. In 19 the source term on the boundary $\partial \Omega_{0, T}$, namely $g^{\varepsilon}$, reads:

$$
g^{\varepsilon}:=g^{\varepsilon}\left(t, x_{1}\right)=e^{\frac{i}{\varepsilon}\left(\underline{\tau} t+\underline{\eta} x_{1}\right)} g\left(t, x_{1}\right)
$$

for a fixed frequency $\underline{\zeta}=(i \underline{\tau}, \underline{\eta}) \in \Upsilon$ and a fixed amplitude $g \in H^{\infty}\left(\Omega_{T}\right)$ satisfying $g_{\mid t \leq 0}=0$.

\footnotetext{
${ }^{5}$ Recall here that from Assumption 3.2 we have $\operatorname{det} A_{2} \neq 0$
} 
We choose for ansatz

$$
u^{\varepsilon} \sim \sum_{n \geq 0} \varepsilon^{n} \sum_{k \in \mathscr{H}} e^{\frac{i}{\varepsilon} \varphi_{k}(t, x)} u_{n, k}^{\varepsilon}(t, x)
$$

where the amplitudes $u_{n, k}^{\varepsilon}$ are in the profile space $\mathscr{P}_{n}$ defined as follows

Definition 4.3 Let $T>0$, $\alpha$ be given by 12 and $X \subset \mathbb{R}^{d}$. For all $n \in \mathbb{N}$ we define

$$
\left.\left.\mathscr{P}_{n}:=\mathscr{P}_{n, T, \alpha}(X)=\left\{u \in H^{\infty}(]-\infty, T\right] \times X\right) \backslash u_{\mid t \leq(K-n) \alpha}=0 \text { for } n \leq K \text { and } u_{\mid t \leq 0}=0 \text { for } n>K\right\} \text {. }
$$

For convenience we denote by $\mathscr{P}_{-1}=\{0\}$ the singleton containing the zero function.

In 20 the phases, namely the $\varphi_{k}(t, x)$, are defined by $\varphi_{k}(t, x):=\underline{\tau} t+\underline{\eta} x_{1}+\xi_{k} x_{2}$, where the $\xi_{k}$ are the roots in the $\xi$-variable of the dispersion relation $\operatorname{det} \mathscr{L}(\underline{\tau}, \underline{\eta}, \xi)=0$ (recall that $\mathscr{L}$ is the symbol of $L(\partial)$ ).

Plugging the ansatz (20) in the interior equation of 19 gives the iterative relation:

$$
\begin{cases}\mathscr{L}\left(\mathrm{d} \varphi_{k}\right) u_{0, k}^{\varepsilon}=0 & \forall k \in \mathscr{H}, \\ i \mathscr{L}\left(\mathrm{d} \varphi_{k}\right) u_{n+1, k}^{\varepsilon}+L(\partial) u_{n, k}^{\varepsilon}=0 & \forall n \geq 0, \forall k \in \mathscr{H} .\end{cases}
$$

If we now inject the ansatz (20) in the boundary conditions of 19 we obtain that for all $n \geq 0$ :

$$
B_{0}\left(\sum_{k \in \mathscr{I}} u_{n, k}^{\varepsilon}+\sum_{\ell \in \mathscr{O}} u_{n, \ell}^{\varepsilon}\right)_{\mid x_{2}=0}=\delta_{n, K+1} g
$$

for the boundary condition on $\partial \Omega_{0}(\delta$, , stands for the Kronecker symbol) and

$$
B_{1}\left(\sum_{k \in \mathscr{I}} e^{\frac{i}{\varepsilon} \xi_{k}} u_{n, k}^{\varepsilon}+\sum_{\ell \in \mathscr{O}} e^{\frac{i}{\varepsilon} \xi_{\ell}} u_{n, \ell}^{\varepsilon}\right)_{\mid x_{2}=1}=0
$$

for the boundary condition on $\partial \Omega_{1}$.

Finally concerning the initial condition it is automatically satisfied if

$$
\forall n \geq 0, \forall k \in \mathscr{H}, u_{n, k}^{\varepsilon} \in \mathscr{P}_{n} .
$$

\subsection{Determination of the leading order amplitudes}

The first equation of 22 written for $n=0$ gives the polarization condition

$$
\forall k \in \mathscr{H}, P^{k} u_{0, k}^{\varepsilon}=u_{0, k}^{\varepsilon},
$$

where we recall that the projection $P^{k}$ is introduceded in Definition 4.1 . Using the fact that for $k \in \mathscr{I}, u_{0, k}^{\varepsilon} \in$ $E^{s}(\underline{\zeta})$ and the decomposition given in Definition 4.1 we write

$$
\begin{array}{r}
\sum_{k \in \mathscr{I}} u_{0, k_{\mid x_{2}=0}}^{\varepsilon}\left(t, x_{1}\right)=\varrho_{0}^{\varepsilon}\left(t, x_{1}\right) e+\nu_{0}^{\varepsilon}\left(t, x_{1}\right) v_{0, *} \\
\text { so that } \forall k \in \mathscr{I}, P^{k} u_{0, k_{\mid x_{2}=0}^{\varepsilon}}^{\varepsilon}=\varrho_{0}^{\varepsilon} e_{k}+\nu_{0}^{\varepsilon} P^{k} v_{0, *}:=\varrho_{0}^{\varepsilon} e_{k}+\nu_{0}^{\varepsilon} v_{0, *, k},
\end{array}
$$

where $e$ is a vector such that $\operatorname{ker} B_{0} \cap E^{s}(\underline{\zeta})=\operatorname{vect}\{e\}$ and $v_{0, *} \in E_{*}^{s}(\underline{\zeta})$.

Also note that in (26), $\varrho_{0}^{\varepsilon}$ and $\nu_{0}^{\varepsilon}$ are scalar functions that inherit the profile space properties ${ }^{6}$ of $u_{0, k}^{\varepsilon}$ and that need to be determined to construct the trace of $\sum_{k \in \mathscr{I}} u_{0, k}^{\varepsilon}$ on $\partial \Omega_{0}$. As we will see the knowledge of these functions is the only thing needed to determine all the amplitudes in the WKB expansion.

Then we multiply in the left hand side the second equation of 22 written for $n=0$ by $Q^{k}$ to obtain

$$
\forall k \in \mathscr{H}, Q^{k} L(\partial) P^{k} u_{0, k}^{\varepsilon}=0,
$$

and we are in position to apply Lax Lemma

\footnotetext{
${ }^{6}$ In fact we will see in the following that we have $\varrho_{0}^{\varepsilon} \in \mathscr{P}_{0}$ and $\nu_{0}^{\varepsilon} \in \mathscr{P}_{-1}$.
} 
Lemma 4.1 (Lax, [Lax57]) Under Assumption 3.1 for all $k \in \mathscr{H}$ we have the relation:

$$
Q^{k} L(\partial) P^{k} u=\left(\partial_{t}+\mathbf{v}_{k} \cdot \nabla_{x}\right) Q^{k} P^{k} u,
$$

where we recall that $\mathbf{v}_{k}$ stands for the group velocity associated to the phase $\varphi_{k}$ introduced in Definition 3.2 .

Consequently if $k \in \mathscr{I}$, from Lax lemma combined with 23) and (25), we are interested in the transport equation:

$$
\forall k \in \mathscr{I}, \begin{cases}\left(\partial_{t}+\mathbf{v}_{k} \cdot \nabla_{x}\right) u_{0, k}^{\varepsilon}=0 & \text { in } \Omega_{T}, \\ B_{0}\left(\sum_{k \in \mathscr{I}} u_{0, k}^{\varepsilon}\right)_{\mid x_{2}=0}=-B_{0}\left(\sum_{\ell \in \mathscr{O}} u_{0, \ell}^{\varepsilon}\right)_{\mid x_{2}=0} & \text { on } \partial \Omega_{0, T}, \\ u_{0, k}^{\varepsilon} \in \mathscr{P}_{0}, & \end{cases}
$$

while for $\ell \in \mathscr{O}$ we consider (using (24) this time):

$$
\forall \ell \in \mathscr{O}, \begin{cases}\left(\partial_{t}+\mathbf{v}_{\ell} \cdot \nabla_{x}\right) u_{0, \ell}^{\varepsilon}=0 & \text { in } \Omega_{T}, \\ B_{1}\left(\sum_{\ell \in \mathscr{O}} e^{\frac{i}{\varepsilon} \xi_{\ell}} u_{0, \ell}^{\varepsilon}\right)_{\mid x_{2}=1}=-B_{1}\left(\sum_{k \in \mathscr{I}} e^{\frac{i}{\varepsilon} \xi_{k}} u_{0, k}^{\varepsilon}\right)_{\mid x_{2}=1} & \text { on } \partial \Omega_{1, T}, \\ u_{0, \ell}^{\varepsilon} \in \mathscr{P}_{0} . & \end{cases}
$$

The equation for outgoing amplitudes is warless indeed we can apply the uniform Kreiss-Lopatinskii condition in the boundary condition on $\partial \Omega_{1, T}$ and then apply the projection $P^{\ell}$ to obtain the desired value of the trace of outgoing amplitudes, namely

$$
\forall \ell \in \mathscr{O}, u_{0, \ell_{\mid x_{2}=1}^{\varepsilon}}^{\varepsilon}=-e^{-\frac{i}{\varepsilon} \xi_{\ell}} P^{\ell} \phi_{1} B_{1} \sum_{k \in \mathscr{I}} e^{\frac{i}{\varepsilon} \xi_{k}} u_{0, k_{\mid x_{2}=1}}^{\varepsilon} .
$$

However we can not use the same trick as it is done in Benb for incoming waves because we do not assume the uniform Kreiss-Lopatinskii condition on the side $\partial \Omega_{0}$ but that we are in the $W R$-class.

Consequently the main issue in the $W R$-class is that the boundary condition of $(27)$ does not provide any information on $\varrho_{0}^{\varepsilon}$ so that the components of the trace on the $e_{k}$ are missing in (27).

Let us assume for a while that $\varrho_{0}^{\varepsilon}$ and $\nu_{0}^{\varepsilon}$ are given functions. Then it is easy to solve 27] by integration along the characteristics. We obtain

$$
\forall k \in \mathscr{I}, u_{0, k}^{\varepsilon}(t, x)=\varrho_{0}^{\varepsilon}\left(t-\frac{1}{\mathbf{v}_{k, 2}} x_{2}, x_{1}-\frac{\mathbf{v}_{k, 1}}{\mathbf{v}_{k, 2}} x_{2}\right) e_{k}+\nu_{0}^{\varepsilon}\left(t-\frac{1}{\mathbf{v}_{k, 2}} x_{2}, x_{1}-\frac{\mathbf{v}_{k, 1}}{\mathbf{v}_{k, 2}} x_{2}\right) v_{0, *, k} .
$$

In order to save some notations in the following we introduce

$$
\mathbf{t}_{\rightarrow}:=\mathbf{t}_{\rightarrow, k}\left(t, x_{2}\right)=t-\frac{1}{\mathbf{v}_{k, 2}} x_{2} \text { and } \mathbf{x}_{\rightarrow}:=\mathbf{x}_{\rightarrow, k}(x)=x_{1}-\frac{\mathbf{v}_{k, 1}}{\mathbf{v}_{k, 2}} x_{2} .
$$

Consequently at this step of the construction the right hand side of 29 is a known function (or depending on $\varrho_{0}^{\varepsilon}$ and $\nu_{0}^{\varepsilon}$ ) and we are free to integrate (28) along the characteristics to obtain the value of the outgoing amplitudes. More precisely for all $\ell \in \mathscr{O}$ we have

$$
\begin{aligned}
u_{0, \ell}^{\varepsilon}(t, x)= & -P^{\ell} \phi_{1} B_{1} \sum_{k \in \mathscr{I}} e^{\frac{i}{\varepsilon}\left(\xi_{k}-\xi_{\ell}\right)} \varrho_{0}^{\varepsilon}\left(t-\frac{1}{\mathbf{v}_{k, 2}}+\frac{1}{\mathbf{v}_{\ell, 2}}\left(1-x_{2}\right), x_{1}-\frac{\mathbf{v}_{k, 1}}{\mathbf{v}_{k, 2}}+\frac{\mathbf{v}_{\ell, 1}}{\mathbf{v}_{\ell, 2}}\left(1-x_{2}\right)\right) e_{k} \\
& -P^{\ell} \phi_{1} B_{1} \sum_{k \in \mathscr{I}} e^{\frac{i}{\varepsilon}\left(\xi_{k}-\xi_{\ell}\right)} \nu_{0}^{\varepsilon}\left(t-\frac{1}{\mathbf{v}_{k, 2}}+\frac{1}{\mathbf{v}_{\ell, 2}}\left(1-x_{2}\right), x_{1}-\frac{\mathbf{v}_{k, 1}}{\mathbf{v}_{k, 2}}+\frac{\mathbf{v}_{\ell, 1}}{\mathbf{v}_{\ell, 2}}\left(1-x_{2}\right)\right) v_{0, *, k} .
\end{aligned}
$$

Once again we introduce the short hand notations

$$
\mathbf{t}_{\leftarrow}:=\mathbf{t}_{\leftarrow, k, \ell}\left(t, x_{2}\right)=t-\frac{1}{\mathbf{v}_{k, 2}}+\frac{1}{\mathbf{v}_{\ell, 2}}\left(1-x_{2}\right) \text { and } \mathbf{x}_{\leftarrow, k, \ell}:=\mathbf{x}_{\leftarrow}(x)=x_{1}-\frac{\mathbf{v}_{k, 1}}{\mathbf{v}_{k, 2}}+\frac{\mathbf{v}_{\ell, 1}}{\mathbf{v}_{\ell, 2}}\left(1-x_{2}\right) .
$$


So we can explicit the right hand side of the boundary condition of 27 in terms of $\varrho_{0}^{\varepsilon}$ and $\nu_{0}^{\varepsilon}$. Indeed we have

$$
B_{0} \sum_{k \in \mathscr{I}} u_{0, k_{\mid x_{2}=0}}^{\varepsilon}=-B_{0} \sum_{\ell \in \mathscr{O}} u_{0, \ell_{\mid x_{2}=0}^{\varepsilon}}^{\varepsilon} \Longrightarrow \nu_{0}^{\varepsilon} v_{0, *}=-\phi_{0, *} B_{0} \sum_{\ell \in \mathscr{O}} u_{0, \ell_{\mid x_{2}=0}^{\varepsilon}},
$$

where we used the fact that the uniform Kreiss-Lopatinskii condition holds on the restricted stable subspace $E_{*}^{s}(\zeta)$.

So finally some computations (see [Benb]) give the following relation between $\varrho_{0}^{\varepsilon}$ and $\nu_{0}^{\varepsilon}$ :

$$
\left(I-\mathcal{S}_{*}^{\varepsilon}\right) \nu_{0}^{\varepsilon} v_{0, *}=\mathcal{S}_{*}^{\varepsilon} \varrho_{0}^{\varepsilon} e,
$$

where we defined for functions $f v$, with $f$ a scalar function depending on $\left(t, x_{1}\right)$ and a fixed vector $v$ :

$$
\left(\mathcal{S}_{*}^{\varepsilon} f v\right)\left(t, x_{1}\right):=\sum_{\ell \in \mathscr{O}} \sum_{k \in \mathscr{I}} e^{\frac{i}{\varepsilon}\left(\xi_{k}-\xi_{\ell}\right)} f\left(t-\alpha_{k, \ell}, x_{1}-\beta_{k, \ell}\right) \phi_{0, *} B_{0} P^{\ell} \phi_{1} B_{1} P^{k} v,
$$

with $\alpha_{k, \ell}:=\frac{1}{\mathbf{v}_{k, 2}}-\frac{1}{\mathbf{v}_{\ell, 2}}>0$ and $\beta_{k, \ell}:=\frac{\mathbf{v}_{k, 1}}{\mathbf{v}_{k, 2}}-\frac{\mathbf{v}_{\ell, 1}}{\mathbf{v}_{\ell, 2}} \in \mathbb{R}$.

Note that up to the restriction of $B_{0}$ to $E_{*}^{s}\left(\underline{\zeta)}\right.$ the definition of the operator $\mathcal{S}_{*}^{\varepsilon}$ is the same as the operator of self-interaction of Benb].

To determine $\nu_{0}^{\varepsilon} v_{0, *}$ in terms of $\varrho_{0}^{\epsilon} e$ it is sufficient to invert the operator $\left(I-\mathcal{S}_{*}^{\varepsilon}\right)$. The main argument in order to do this is that we are in finite time and that the terms in the Neumann series expansion of $\left(I-\mathcal{S}_{*}^{\varepsilon}\right)$ reads essentially $7^{7}$ under the form:

$$
\left(\left(\mathcal{S}_{*}^{\varepsilon}\right)^{j} f v\right)\left(t, x_{1}\right)=\sum_{l=0}^{j} f\left(t-A_{l}, x_{1}-B_{l}\right) R^{\varepsilon, l} v
$$

where the $R^{\varepsilon, l}$ are matrices and where the coefficients $A_{l}>0$ and (resp. $B_{l} \in \mathbb{R}$ ) are sum of terms in $\left\{\alpha_{k, \ell}\right\}_{k, \ell}$ (resp. $\left.\left\{\beta_{k, \ell}\right\}_{k, \ell}\right)$ with $l$ elements.

From the profile space definition $\varrho_{0}^{\varepsilon}$ is zero under the time threshold $\alpha K \leq \alpha_{k, \ell} K$ for all $k \in \mathscr{I}, \ell \in \mathscr{O}$ combined with the fact that $A_{l}$ is increasing with $l$ (because the $\alpha_{k, \ell}$ are positive) it follows that $\left(\left(\mathcal{S}_{*}^{\varepsilon}\right)^{j} \varrho_{0}^{\varepsilon}\right)$ is zero up to some $j$. Consequently the Neumann serie expansion contains a finite number of terms so that $\left(I-\mathcal{S}_{*}^{\varepsilon}\right)^{-1}$ is well-defined.

Moreover in the case of $\nu_{0}^{\varepsilon} v_{0, *}$ we have a nice simplification. Indeed:

$$
\nu_{0}^{\varepsilon} v_{0, *}=\sum_{j=1}^{\infty}\left(\mathcal{S}^{\varepsilon}\right)^{j} \varrho_{0}^{\varepsilon} e .
$$

But recall that $\varrho_{0}^{\varepsilon} \in \mathscr{P}_{0}$ so that we have $\varrho_{0}^{\varepsilon}\left(t, x_{1}\right)=0$ as soon as $t \leq \alpha K$. Consequently we have:

$$
t-\alpha_{k, \ell} \leq T-\alpha \leq \alpha\left(\left[\frac{T}{\alpha}\right]+1\right)-\alpha=\alpha K
$$

So all the terms in the Neumann series expansion vanish and consequently we have $\nu_{0}^{\varepsilon} v_{0, *} \equiv 0$.

Remark This observation is totally natural. Indeed, the loss of derivatives studied in CG10 for hyperbolic boundary value problems in the $W R$-class comes from the component of $u_{0, k}^{\varepsilon}$ lying on vect $\{e\}$ but not from the one on $E_{*}^{s}(\underline{\zeta})$ so we recover that $\nu_{0}^{\varepsilon} v_{0, *} \equiv 0$ on the time scale $t \leq \alpha K$.

It follows that we can use this cancellation in $(26)$ and $(32)$ to express all the leading order amplitudes in terms of $\varrho_{0}^{\varepsilon}$ only.

The aim of the following is thus to determine $\varrho_{0}^{\varepsilon}$ in order to complete the construction of the leading order term.

\footnotetext{
${ }^{7}$ Considering that the precise value of the terms in the expansion is not really necessary for the remaining of the proof we do not give them explicitly here.
} 


\subsubsection{Determination of $\varrho_{0}^{\varepsilon}$}

The method to determine the unknown $\varrho_{0}^{\varepsilon}$ described here follows the one of [CG10] but it has to be adapted to multiple losses of derivative as in Ben17. The only equation of the WKB cascade in which $\varrho_{0}^{\varepsilon}$ appears is the boundary condition of order one on the side $\partial \Omega_{0, T}$. This condition can be written in the following form:

$$
B_{0} \sum_{k \in \mathscr{I}} P^{k} u_{1, k_{\mid x_{2}=0}^{\varepsilon}}^{\varepsilon}+B_{0} \sum_{k \in \mathscr{I}}\left(I-P^{k}\right) u_{1, k_{\mid x_{2}=0}^{\varepsilon}}^{\varepsilon}+B_{0} \sum_{\ell \in \mathscr{O}} u_{1, \ell_{\mid x_{2}=0}}^{\varepsilon}=\delta_{K, 0} g .
$$

Taking the inner product of 37 ) with the vector $b$ introduced in Definition 4.2 (so that the first term in the left hand side of (37) vanishes) then gives:

$$
b \cdot B_{0} \sum_{k \in \mathscr{I}}\left(I-P^{k}\right) u_{1, k_{\mid x_{2}=0}}^{\varepsilon}+b \cdot B_{0} \sum_{\ell \in \mathscr{O}} P^{\ell} u_{1, \ell_{\mid x_{2}=0}^{\varepsilon}}^{\varepsilon}+b \cdot B_{0} \sum_{\ell \in \mathscr{O}}\left(I-P^{\ell}\right) u_{1, \ell_{\mid x_{2}=0}^{\varepsilon}}=\delta_{K, 0} b \cdot g
$$

and we shall explicit each terms in the left hand side of $(38)$ in terms of $\varrho_{0}^{\varepsilon}$.

Firstly we deal with the terms involving the unpolarized part of the amplitudes. Composing the second equation of (22) by $R^{k}$ (see Definition 4.1) shows that the unpolarized part of the amplitude of order one depends on the amplitude of the leading order amplitude. Indeed we have:

$$
\forall k \in \mathscr{H},\left(I-P^{k}\right) u_{1, k}^{\varepsilon}=i R^{k} L(\partial) u_{0, k}^{\varepsilon}
$$

We now use the following Lemma

Lemma 4.2 ([CG10 $]$ ) For all $k \in \mathscr{H}$, we have that $R^{k} A_{2} P^{k}=0$.

Moreover if $d=2$ we also have $R^{k} A_{1} P^{k}=Q^{k} A_{1} R^{k}=-\frac{\tau}{\underline{\eta}} R^{k} P^{k}$.

We first compute $\left(I-P^{\ell}\right) u_{1, \ell}^{\varepsilon}$ for $\ell \in \mathscr{O}$ using 39 . We have from 32 (in which we use the fact that $\left.\nu_{0}^{\varepsilon} v_{0, *} \equiv 0\right)$ :

$$
i R^{\ell} L(\partial) u_{0, \ell}^{\varepsilon}=-i R^{\ell} P^{\ell} \phi_{1} B_{1} \sum_{k \in \mathscr{I}} e^{\frac{i}{\varepsilon}\left(\xi_{k}-\xi_{\ell}\right)} \partial_{t} \varrho_{0}^{\varepsilon}\left(\mathbf{t}_{\leftarrow}, \mathbf{x}_{\leftarrow}\right) e_{k}-i \underbrace{R^{\ell} A_{1} P^{\ell}}_{=-\underline{\underline{\tau}} R^{\ell} P^{\ell}} \phi_{1} B_{1} \sum_{k \in \mathscr{I}} e^{\frac{i}{\varepsilon}\left(\xi_{k}-\xi_{\ell}\right)} \partial_{1} \varrho_{0}^{\varepsilon}\left(\mathbf{t}_{\leftarrow}, \mathbf{x}_{\leftarrow}\right) e_{k} .
$$

So for all $\ell \in \mathscr{O}$

$$
\left(I-P^{\ell}\right) u_{1, \ell}^{\varepsilon}=-i R^{\ell} P^{\ell} \phi_{1} B_{1} \sum_{k \in \mathscr{I}} e^{\frac{i}{\varepsilon}\left(\xi_{k}-\xi_{\ell}\right)}\left(\mathcal{T} \varrho_{0}^{\varepsilon}\right)\left(\mathbf{t}_{\leftarrow}, \mathbf{x}_{\leftarrow}\right) e_{k}
$$

where the transport operator on the boundary $\mathcal{T}$ is defined by

$$
\mathcal{T}:=\partial_{t}-\frac{\tau}{\underline{\eta}} \partial_{1}
$$

The same kind of computations shows that the unpolarized part of the incoming waves are given by:

$$
\forall k \in \mathscr{I},\left(I-P^{k}\right) u_{1, k}^{\varepsilon}=i\left(\mathcal{T} \varrho_{0}^{\varepsilon}\right)\left(\mathbf{t}_{\rightarrow}, \mathbf{x}_{\rightarrow}\right) R^{k} e_{k}
$$

This completes the determination of unpolarized amplitudes in (38) and its remains to determine the polarized part of the outgoing amplitudes. In order to do so we essentially reiterate the computations made to construct the amplitudes of order zero up to two non-trivial modifications:

- the amplitudes of order one are not polarized any more.

- We need to decompose the (unknown) polarized part of the trace $\sum_{k \in \mathscr{I}} P^{k} u_{1, k_{\mid x_{2}=0}}$ on the subspaces vect $\{e\}$ and $E_{*}^{s}(\underline{\zeta})$ by introducing two new unknowns scalar functions $\varrho_{1}^{\varepsilon}$ and $\nu_{1}^{\varepsilon}$ (see [26).

However we will have $\nu_{1}^{\varepsilon} \in \mathscr{P}_{0}$, this time, so the terms involving $\nu_{1}^{\varepsilon}$ can not be neglected as the ones involving $\nu_{0}^{\varepsilon}$ in the previous discussion. 
Let us stress that these two modifications do not modify in an essential way the loss of derivatives process (and more precisely the time of appearance of the losses). Indeed this process only depends on $e$ and the group velocities (which are given by the hyperbolic boundary value problem).

As a consequence compared to the equations on the leading order amplitudes the previously exposed modifications will more or less only give rise to the same equations up to some (possibly complicated) extra terms to take into account, however the main idea remains the one exposed in Section 2.

Multiply the second equation of 22 by $Q^{k}$ for $k \in \mathscr{H}$ and using Lax Lemma shows that the polarized part of the amplitudes solves the following transport equations:

$$
\forall k \in \mathscr{I},\left\{\begin{array}{l}
\left(\partial_{t}+\mathbf{v}_{k} \cdot \nabla_{x}\right) Q^{k} P^{k} u_{1, k}^{\varepsilon}=-Q^{k} L(\partial)\left(I-P^{k}\right) u_{1, k}^{\varepsilon}, \\
B_{0} \sum_{k \in \mathscr{I}} P^{k} u_{1, k_{\mid x_{2}=0}}^{\varepsilon}=-B_{0} \sum_{\ell \in \mathscr{O}} u_{1, \ell_{\mid x_{2}=0}=0}^{\varepsilon}-B_{0} \sum_{k \in \mathscr{I}}\left(I-P^{k}\right) u_{1, k_{\mid x_{2}=0}^{\varepsilon}}, \\
u_{1, k_{\mid t \leq(K-1) \alpha}}^{\varepsilon}=0
\end{array}\right.
$$

and

$$
\forall \ell \in \mathscr{O},\left\{\begin{array}{l}
\left(\partial_{t}+\mathbf{v}_{\ell} \cdot \nabla_{x}\right) Q^{\ell} P^{\ell} u_{1, \ell}^{\varepsilon}=-Q^{\ell} L(\partial)\left(I-P^{\ell}\right) u_{1, \ell}^{\varepsilon}, \\
B_{1} \sum_{\ell \in \mathscr{O}} e^{\frac{i}{\varepsilon} \xi_{\ell}} P^{\ell} u_{1, \ell_{\mid x_{2}=1}^{\varepsilon}}^{\varepsilon}=-B_{1} \sum_{k \in \mathscr{I}} e^{\frac{i}{\varepsilon} \xi_{k}} u_{1, k_{\mid x_{2}=1}^{\varepsilon}}^{\varepsilon}-B_{1} \sum_{\ell \in \mathscr{O}} e^{\frac{i}{\varepsilon} \xi_{\ell}}\left(I-P^{\ell}\right) u_{1, \ell_{\mid x_{2}=1}^{\varepsilon}}^{\varepsilon}, \\
u_{1, \ell_{\mid t \leq(K-1) \alpha}}^{\varepsilon}=0,
\end{array}\right.
$$

and we begin by the resolution of (43).

Firstly we shall make the interior term explicit with respect to $\varrho_{0}^{\varepsilon}$. From 42 and Lemma 4.2 , we deduce that for all $k \in \mathscr{I}$ :

$$
\begin{aligned}
-Q^{k} L(\partial)\left(I-P^{k}\right) u_{1, k}^{\varepsilon}= & -i Q^{k}\left(L(\partial)\left(\mathcal{T} \varrho_{0}^{\varepsilon}\right)\left(\mathbf{t}_{\rightarrow}, \mathbf{x}_{\rightarrow}\right) R^{k} e_{k}\right) \\
= & -i\left(\partial_{t t}^{2} \varrho_{0}^{\varepsilon}-\frac{\underline{\tau}}{\underline{\eta}} \partial_{t 1}^{2} \varrho_{0}^{\varepsilon}\right)\left(\mathbf{t}_{\rightarrow}, \mathbf{x}_{\rightarrow}\right) Q^{k} R^{k} e_{k} \\
& -i\left(\partial_{1 t}^{2} \varrho_{0}^{\varepsilon}-\frac{\tau}{\underline{\eta}} \partial_{11}^{2} \varrho_{0}^{\varepsilon}\right)\left(\mathbf{t}_{\rightarrow}, \mathbf{x}_{\rightarrow}\right) \underbrace{Q^{k} A_{1} R^{k} e_{k}}_{=-\frac{\tau}{\underline{\underline{T}}} Q^{k} R^{k} e_{k}} \\
= & -i\left(\mathcal{T}^{2} \varrho_{0}^{\varepsilon}\right)\left(\mathbf{t}_{\rightarrow}, \mathbf{x}_{\rightarrow}\right) Q^{k} R^{k} e_{k} .
\end{aligned}
$$

Once again, because of the degeneracy of the uniform Kreiss-Lopatinskii condition on $\partial \Omega_{0}$, the boundary condition of 430 is not sufficient to determine $P^{k} u_{1, k_{\mid x_{2}=0}}^{\varepsilon}$. So we introduce the notation:

$$
\forall k \in \mathscr{I}, P^{k} u_{1, k_{\mid x_{2}=0}^{\varepsilon}}^{\varepsilon}\left(t, x_{1}\right):=\varrho_{1}^{\varepsilon}\left(t, x_{1}\right) e_{k}+\nu_{1}^{\varepsilon}\left(t, x_{1}\right) v_{1, *, k},
$$

where $v_{1, *, k}:=P^{k} v_{1, *}$ with $v_{1, *} \in E_{*}^{s}(\underline{\zeta})$ and $\varrho_{1}^{\varepsilon} \in \mathscr{P}_{1}, \nu_{1}^{\varepsilon} \in \mathscr{P}_{0}$ are two (unknown) scalar functions.

Noticing that the source term in the interior of the transport equation (43) lies along the characteristics we obtain that

$$
P^{k} u_{1, k}^{\varepsilon}(t, x)=\varrho_{1}^{\varepsilon}\left(\mathbf{t}_{\rightarrow}, \mathbf{x}_{\rightarrow}\right) e_{k}+\nu_{1}^{\varepsilon}\left(\mathbf{t}_{\rightarrow}, \mathbf{x}_{\rightarrow}\right) v_{1, *, k}-i \frac{x_{2}}{\mathbf{v}_{k, 2}}\left(\mathcal{T}^{2} \varrho_{0}^{\varepsilon}\right)\left(\mathbf{t}_{\rightarrow}, \mathbf{x}_{\rightarrow}\right) \widetilde{Q}^{k} Q^{k} R^{k} e_{k},
$$

where $\widetilde{Q}^{k}=\left(Q_{\mid \operatorname{Ran} P^{k}}^{k}\right)^{-1}$.

We now turn to the resolution of (44) in terms of $\varrho_{0}^{\varepsilon}, \varrho_{1}^{\varepsilon}$ and $\nu_{1}^{\varepsilon}$. In order to do so we shall explicit the dependency of the source terms with respect to these functions. Firstly for the interior source term, reiterating essentially the same computations as for 45, we have:

$$
-Q^{\ell} L(\partial)\left(I-P^{\ell}\right) u_{1, \ell}^{\varepsilon}=i Q^{\ell} R^{\ell} P^{\ell} \phi_{1} B_{1} \sum_{k \in \mathscr{I}} e^{\frac{i}{\varepsilon}\left(\xi_{k}-\xi_{\ell}\right)}\left(\mathcal{T}^{2} \varrho_{0}^{\varepsilon}\right)\left(\mathbf{t}_{\leftarrow}, \mathbf{x}_{\leftarrow}\right) e_{k} .
$$


Then (40), 42] and 47] and the uniform Kreiss-Lopatinskii on the side $\partial \Omega_{1, T}$ give

$$
\begin{aligned}
P^{\ell} u_{1, \ell_{\mid x_{2}=1}^{\varepsilon}}^{\varepsilon}= & -\sum_{k \in \mathscr{I}} e^{\frac{i}{\varepsilon}\left(\xi_{k}-\xi_{\ell}\right)} \varrho_{1}^{\varepsilon}\left(t-\frac{1}{\mathbf{v}_{k, 2}}, x_{1}-\frac{\mathbf{v}_{k, 1}}{\mathbf{v}_{k, 2}}\right) P^{\ell} \phi_{1} B_{1} e_{k} \\
& -\sum_{k \in \mathscr{I}} e^{\frac{i}{\varepsilon}\left(\xi_{k}-\xi_{\ell}\right)} \nu_{1}^{\varepsilon}\left(t-\frac{1}{\mathbf{v}_{k, 2}}, x_{1}-\frac{\mathbf{v}_{k, 1}}{\mathbf{v}_{k, 2}}\right) P^{\ell} \phi_{1} B_{1} v_{1, *, k} \\
& +i \sum_{k \in \mathscr{I}} \frac{e^{\frac{i}{\varepsilon}\left(\xi_{k}-\xi_{\ell}\right)}}{\mathbf{v}_{k, 2}}\left(\mathcal{T}^{2} \varrho_{0}^{\varepsilon}\right)\left(t-\frac{1}{\mathbf{v}_{k, 2}}, x_{1}-\frac{\mathbf{v}_{k, 1}}{\mathbf{v}_{k, 2}}\right) P^{\ell} \phi_{1} B_{1} \widetilde{Q}^{k} Q^{k} R^{k} e_{k} \\
& -i \sum_{k \in \mathscr{I}} e^{\frac{i}{\varepsilon}\left(\xi_{k}-\xi_{\ell}\right)}\left(\mathcal{T} \varrho_{0}^{\varepsilon}\right)\left(t-\frac{1}{\mathbf{v}_{k, 2}}, x_{1}-\frac{\mathbf{v}_{k, 1}}{\mathbf{v}_{k, 2}}\right) P^{\ell} \phi_{1} B_{1} R^{k} e_{k} \\
& +i \sum_{\ell^{\prime} \in \mathscr{O}} \sum_{k \in \mathscr{I}} e^{\frac{i}{\varepsilon}\left(\xi_{k}-\xi_{\ell}\right)}\left(\mathcal{T} \varrho_{0}^{\varepsilon}\right)\left(t-\frac{1}{\mathbf{v}_{k, 2}}, x_{1}-\frac{\mathbf{v}_{k, 1}}{\mathbf{v}_{k, 2}}\right) P^{\ell} \phi_{1} B_{1} R^{\ell^{\prime}} P^{\ell^{\prime}} \phi_{1} B_{1} e_{k} .
\end{aligned}
$$

Consequently we are in position to solve the transport equation (44) and we obtain:

$$
\begin{aligned}
P^{\ell} u_{1, \ell}^{\varepsilon}= & -\sum_{k \in \mathscr{I}} e^{\frac{i}{\varepsilon}\left(\xi_{k}-\xi_{\ell}\right)} \varrho_{1}^{\varepsilon}\left(\mathbf{t}_{\leftarrow}, \mathbf{x}_{\leftarrow}\right) P^{\ell} \phi_{1} B_{1} e_{k} \\
& -\sum_{k \in \mathscr{I}} e^{\frac{i}{\varepsilon}\left(\xi_{k}-\xi_{\ell}\right)} \nu_{1}^{\varepsilon}\left(\mathbf{t}_{\leftarrow}, \mathbf{x}_{\leftarrow}\right) P^{\ell} \phi_{1} B_{1} v_{1, *, k} \\
& +i \sum_{k \in \mathscr{I}} \frac{e^{\frac{i}{\varepsilon}\left(\xi_{k}-\xi_{\ell}\right)}}{\mathbf{v}_{k, 2}}\left(\mathcal{T}^{2} \varrho_{0}^{\varepsilon}\right)\left(\mathbf{t}_{\leftarrow}, \mathbf{x}_{\leftarrow}\right) P^{\ell} \phi_{1} B_{1} \widetilde{Q}^{k} Q^{k} R^{k} e_{k} \\
& -i \sum_{k \in \mathscr{I}} e^{\frac{i}{\varepsilon}\left(\xi_{k}-\xi_{\ell}\right)}\left(\mathcal{T} \varrho_{0}^{\varepsilon}\right)\left(\mathbf{t}_{\leftarrow}, \mathbf{x}_{\leftarrow}\right) P^{\ell} \phi_{1} B_{1} R^{k} e_{k} \\
& +i \sum_{\ell^{\prime} \in \mathscr{O}} \sum_{k \in \mathscr{I}} e^{\frac{i}{\varepsilon}\left(\xi_{k}-\xi_{\ell}\right)}\left(\mathcal{T} \varrho_{0}^{\varepsilon}\right)\left(\mathbf{t}_{\leftarrow}, \mathbf{x}_{\leftarrow}\right) P^{\ell} \phi_{1} B_{1} R^{\ell^{\prime}} P^{\ell^{\prime}} \phi_{1} B_{1} e_{k} \\
& -i \frac{1-x_{2}}{\mathbf{v}_{\ell, 2}} \sum_{k \in \mathscr{I}} e^{\frac{i}{\varepsilon}\left(\xi_{k}-\xi_{\ell}\right)}\left(\mathcal{T}^{2} \varrho_{0}^{\varepsilon}\right)\left(\mathbf{t}_{\leftarrow}, \mathbf{x}_{\leftarrow}\right) \widetilde{Q}^{\ell} Q^{\ell} R^{\ell} P^{\ell} \phi_{1} B_{1} e_{k}
\end{aligned}
$$

where the first terms in the right hand side of (48) comes from the transport of the boundary source term, the last one comes from the transport of the interior source term (which lies along the characteristics).

Consequently the trace of $P^{\ell} u_{1, \ell}^{\varepsilon}$ on the side $\partial \Omega_{0, T}$ is a sum of functions evaluated in $\left(t-\alpha_{k, \ell}, x_{1}-\beta_{k, \ell}\right)$. However by definition of the profile space $\mathscr{P}_{0}$ we have that $\varrho_{0}^{\varepsilon}\left(t-\alpha_{k, \ell}, \cdot-\beta_{k, \ell}\right)=0$ for all $t \leq T$ and for all $(k, \ell) \in \mathscr{I} \times \mathscr{O}$. So all the terms involving $\varrho_{0}^{\varepsilon}$ in 49 can in fact be neglected and we obtain that

$$
\begin{aligned}
\sum_{\ell \in \mathscr{O}} P^{\ell} u_{1, \ell_{\mid x_{2}=0}}^{\varepsilon}= & -\sum_{k \in \mathscr{I}} \sum_{\ell \in \mathscr{O}} e^{\frac{i}{\varepsilon}\left(\xi_{k}-\xi_{\ell}\right)} \varrho_{1}^{\varepsilon}\left(t-\alpha_{k, \ell}, x_{1}-\beta_{k, \ell}\right) P^{\ell} \phi_{1} B_{1} e_{k} \\
& -\sum_{k \in \mathscr{I}} \sum_{\ell \in \mathscr{O}} e^{\frac{i}{\varepsilon}\left(\xi_{k}-\xi_{\ell}\right)} \nu_{1}^{\varepsilon}\left(t-\alpha_{k, \ell}, x_{1}-\beta_{k, \ell}\right) P^{\ell} \phi_{1} B_{1} v_{1, *, k} .
\end{aligned}
$$

At this step of the discussion all the terms in 38 have been explicited in terms of $\varrho_{0}^{\varepsilon}$, $\varrho_{1}^{\varepsilon}$ and $\nu_{1}^{\varepsilon}$. So from 40, 42 and (50), 38) rewrites (note that the contribution of the $\left(I-P^{\ell}\right) u_{1, \ell}^{\varepsilon}$ is zero):

$$
\begin{aligned}
\left(\mathcal{T} \varrho_{0}^{\varepsilon}\right)\left(t, x_{1}\right)= & \delta_{K, 0} c b \cdot g+c b \cdot B_{0} \sum_{k \in \mathscr{I}} \sum_{\ell \in \mathscr{O}} e^{\frac{i}{\varepsilon}\left(\xi_{k}-\xi_{\ell}\right)} \varrho_{1}^{\varepsilon}\left(t-\alpha_{k, \ell}, x_{1}-\beta_{k, \ell}\right) P^{\ell} \phi_{1} B_{1} e_{k} \\
& +c b \cdot B_{0} \sum_{k \in \mathscr{I}} \sum_{\ell \in \mathscr{O}} e^{\frac{i}{\varepsilon}\left(\xi_{k}-\xi_{\ell}\right)} \nu_{1}^{\varepsilon}\left(t-\alpha_{k, \ell}, x_{1}-\beta_{k, \ell}\right) P^{\ell} \phi_{1} B_{1} P^{k} v_{1, *}
\end{aligned}
$$

with $c:=\frac{1}{i b \cdot B_{0} \sum_{k \in \mathscr{I}} R^{k} e_{k}} \neq 0$ so that we can express $\varrho_{0}^{\varepsilon}$ in terms of $\varrho_{1}^{\varepsilon}$ and $\nu_{1}^{\varepsilon}$ by solving a transport equation along the boundary. 
Now we shall determine $\nu_{1}^{\varepsilon} v_{1, *}$ in terms of $\varrho_{1}^{\varepsilon}$ and $\varrho_{0}^{\varepsilon}$. We consider 23 written for $n=1$. This condition reads:

$$
\forall k \in \mathscr{I}, \nu_{1}^{\varepsilon} v_{1, *, k}=\delta_{K, 0} P^{k} \phi_{0, *} g-P^{k} \phi_{0, *} B_{0} \sum_{\ell \in \mathscr{O}} u_{1, \ell_{\mid x_{2}=0}}-P^{k} \phi_{0, *} B_{0} \sum_{k^{\prime} \in \mathscr{I}}\left(I-P^{k^{\prime}}\right) u_{1, k_{\mid x_{2}=0}^{\prime}} .
$$

Using (42), 40, and (50) combined with the fact that $\varrho_{0}^{\varepsilon} \in \mathscr{P}_{0}, 52$ becomes

$$
\left(\left(I-\mathcal{S}_{*}^{\varepsilon}\right) \nu_{1}^{\varepsilon} v_{1, *}\right)\left(t, x_{1}\right)=\delta_{K, 0} \phi_{0, *} g\left(t, x_{1}\right)+\left(\mathcal{S}_{*}^{\varepsilon} \varrho_{1}^{\varepsilon} e\right)\left(t, x_{1}\right)-i \phi_{0, *} B_{0} \sum_{k \in \mathscr{I}}\left(\mathcal{T} \varrho_{0}^{\varepsilon}\right)\left(t, x_{1}\right) R^{k} e_{k},
$$

and once again we determine $\nu_{1}^{\varepsilon} v_{1, *}$ from the Neumann series expansion, from the definition of the profile space we have:

$$
\begin{aligned}
\nu_{1}^{\varepsilon}\left(t, x_{1}\right) v_{1, *} & =\delta_{K, 0} \sum_{j \geq 0}\left(\mathcal{S}_{*}^{\varepsilon}\right)^{j} \phi_{0, *} g+\sum_{j \geq 1}\left(\mathcal{S}_{*}^{\varepsilon}\right)^{j} \varrho_{1}^{\varepsilon} e-i \sum_{j \geq 0}\left(\mathcal{S}_{*}^{\varepsilon}\right)^{j} \sum_{k \in \mathscr{I}}\left(\mathcal{T} \varrho_{0}^{\varepsilon}\right) \phi_{0, *} B_{0} R^{k} e_{k} \\
& =\delta_{K, 0} \phi_{0, *} g+\mathcal{S}_{*}^{\varepsilon} \varrho_{1}^{\varepsilon}-i \sum_{k \in \mathscr{I}}\left(\mathcal{T} \varrho_{0}^{\varepsilon}\right) \phi_{0, *} B_{0} R^{k} e_{k} .
\end{aligned}
$$

This equation determines $\nu_{1}^{\varepsilon} v_{1, *} \in \mathscr{P}_{0}^{8}$ in terms of the (still) unknowns functions $\varrho_{0}^{\varepsilon}$ and $\varrho_{1}^{\varepsilon}$.

An interesting point is that in (51) we are not interested in $\nu_{1}^{\varepsilon} v_{1, *}$ but in the $\nu_{1}^{\varepsilon}\left(t-\alpha_{k, \ell}, x_{1}-\beta_{k, \ell}\right) v_{1, *}$ which are known to be zero from (54). As a consequence (51) reads:

$$
\left(\mathcal{T} \varrho_{0}^{\varepsilon}\right)\left(t, x_{1}\right)=\delta_{K, 0} c b \cdot g+c b \cdot B_{0} \sum_{k \in \mathscr{I}} \sum_{\ell \in \mathscr{O}} e^{\frac{i}{\varepsilon}\left(\xi_{k}-\xi_{\ell}\right)} \varrho_{1}^{\varepsilon}\left(t-\alpha_{k, \ell}, x_{1}-\beta_{k, \ell}\right) P^{\ell} \phi_{1} B_{1} e_{k},
$$

so we have determined $\varrho_{0}^{\varepsilon}$ in terms of $\varrho_{1}^{\varepsilon}$.

In particular, if $K=0$ (which is equivalent to impose that the information carried by the source term can not perform a full reflection) (55) reads:

$$
\left(\mathcal{T} \varrho_{0}^{\varepsilon}\right)\left(t, x_{1}\right)=c b \cdot g,
$$

which is nothing but the transport equation on the boundary find in CG10. Consequently if $K=0$ then the construction of the $u_{0, k}^{\varepsilon}, k \in \mathscr{H}$ is complete because $\varrho_{0}^{\varepsilon}$ is easily determinable from the resolution of the boundary transport equation.

A consequence of (55) is that to construct $\varrho_{0}^{\varepsilon}$ we should construct $\varrho_{1}^{\varepsilon}$. Also note that in (55), $\varrho_{0}^{\varepsilon}$ depends on $\varrho_{1}^{\varepsilon}$ evaluated at a time scale large enough to ensure that the wave packets have perform a full reflection.

This means, in particular, that the terms carrying the $(K+1)^{t h}$ loss of derivative come from the term carrying the $K^{\text {th }}$ loss which have made a complete regeneration.

For future purpose for all $n \geq 1$ let

$$
\sum_{k \in \mathscr{I}} P^{k} u_{n, k_{\mid x_{2}=0}^{\varepsilon}}^{\varepsilon}\left(t, x_{1}\right)=\varrho_{n}^{\varepsilon}\left(t, x_{1}\right) e+\nu_{n}^{\varepsilon} v_{n, *},
$$

where $v_{n, *} \in E^{s, *}(\underline{\zeta})$ and where $\varrho_{n}^{\varepsilon} \in \mathscr{P}_{n}, \nu_{n}^{\varepsilon} \in \mathscr{P}_{n-1}$. We also define for all $k \in \mathscr{I}, v_{n, *, k}:=P^{k} v_{n, *}$.

Then with these new notations and the previous remarks the remaining of the construction is clear. We reiterate essentially the same kind of computations to obtain an equation giving $\varrho_{1}^{\varepsilon}$ in terms of $\varrho_{2}^{\varepsilon}$ at a time scale larger than the minimal time to perform a full regeneration of the phases and so on.

So an iterative process will give an equation of the form:

$$
\varrho_{0}^{\varepsilon}\left(t, x_{1}\right)=F_{0}(g)+F_{-1}\left(\varrho_{K+1}^{\varepsilon}\right),
$$

\footnotetext{
${ }^{8}$ It is clear from (54) that $\nu_{1}^{\varepsilon} v_{1, *} \in \mathscr{P}_{0}$. Indeed the last two terms in the right hand side of 54 are in $\mathscr{P}_{0}$. Then the first term in the right hand side of 54 is by definition in $\mathscr{P}_{K}$. However the Kronecker symbol implies that it is non zero if and only if $K=0$.
} 
where $F_{0}(g)$ only depends on the source term $g$ (evaluated on time scales large enough to take into account $K$ full regeneration) and where $F_{-1}\left(\varrho_{K+1}^{\varepsilon}\right)$ only depends on $\varrho_{K+1}^{\varepsilon}$ (evaluated on time scales large enough to take into account $K+1$ full regeneration). However by definition of the profile space $\varrho_{K+1}^{\varepsilon} \in \mathscr{P}_{K+1}=\mathscr{P}_{K}$ so that $F_{-1}\left(\varrho_{K+1}^{\varepsilon}\right)$ is zero and (57) determines $\varrho_{0}^{\varepsilon}$ in terms of the source term $g$ only. Using this expression in (30) and (32) then completes the construction of the $u_{0, k}^{\varepsilon}$ for $k \in \mathscr{H}$.

However, let us point here that unfortunately some technical details complicate the sketch of proof.

- Firstly in all the computations exposed so far we used the fact that $\nu_{0}^{\varepsilon} \equiv 0$ which implies non trivial simplifications. This will not be true any more for $\nu_{n}^{\varepsilon}$ as soon as $n \geq 1$ (recall that from (54), $\nu_{1}^{\varepsilon}$ is not zero for $\alpha K \leq t \leq(\alpha+1) K)$.

As a matter of fact the expressions determining $\left(I-P^{k}\right) u_{n, k}^{\varepsilon},\left(I-P^{\ell}\right) u_{n, \ell}^{\varepsilon}$ and $P^{\ell} u_{n, \ell}^{\varepsilon}$ will include some extra terms compared to (40), 42) and 49) when $n>1$.

- A direct consequence of the previous remark is that the equation determining $\varrho_{n}^{\varepsilon}$ in terms of $\varrho_{n+1}^{\varepsilon}$ is more complicated than (51) as soon as $n \geq 1$ (indeed some extra terms are expected).

However a much more annoying point is that to pass from (51) to 55 we used in a non trivial way that the contribution of the terms involving $\nu_{1}^{\varepsilon}$ in the right hand side of (51). This will not be true any more as soon as $n>1$.

So that because of (52) we expect that (compared (51)) the equation determining $\varrho_{n}^{\varepsilon}$ has in its right hand side a term involving $\varrho_{n}^{\varepsilon}$ that comes from the contribution of $\nu_{n+1}^{\varepsilon}$.

What saves the day is that these terms depend on $\varrho_{n}^{\varepsilon}$ but only on time scales large enough to perform a full regeneration. Consequently the new equation determining $\varrho_{n}^{\varepsilon}$ takes the form

$$
\left(I-\mathscr{R}_{n}^{\varepsilon}\right)\left(\mathcal{T} \varrho_{n}^{\varepsilon}\right)=F_{n}(g)+F_{n-1}\left(\varrho_{n+1}^{\varepsilon}\right),
$$

where $F_{n}(g)$ only depend on the source term, $F_{n-1}\left(\varrho_{n+1}^{\varepsilon}\right)$ only depends on $\varrho_{n+1}^{\varepsilon}$ and $\mathscr{R}_{n}^{\varepsilon}$ is an operator evaluating on times larger than the minimal time needed to perform a full regeneration of the phases. So we can solve $(58)$ by taking the Neumann series expansion of the right hand side to obtain a relation giving $\varrho_{n}^{\varepsilon}$ in terms of $\varrho_{n+1}^{\varepsilon}$ and the iterative argument described to obtain the expected equation (57) applies.

\subsubsection{The general equation on $\varrho_{n}^{\varepsilon}, \varrho_{n+1}^{\varepsilon}, \nu_{n}^{\varepsilon}, \nu_{n+1}^{\varepsilon}$ for $n \geq 1$}

The general equation on $\varrho_{n}^{\varepsilon}, \varrho_{n+1}^{\varepsilon}, \nu_{n}^{\varepsilon}, \nu_{n+1}^{\varepsilon}$ for $n \geq 1$ In this paragraph we essentially reiterate the computations performed in Section 4.3.1 to obtain an equation linking the unknowns components of the trace namely the $\varrho_{n}^{\varepsilon}, \varrho_{n+1}^{\varepsilon}, \nu_{n}^{\varepsilon}, \nu_{n+1}^{\varepsilon}$ for $n \geq 1$.

We however have to take into account the influence of the component $\nu_{n}^{\varepsilon} v_{n, *}$ and the fact that the amplitudes $u_{n, k}^{\varepsilon}, k \in \mathscr{H}$ are not polarized any more.

The main result of this paragraph is the following proposition:

Proposition 4.1 For all $n \geq 1$, for $k \in \mathscr{I}$ and $\ell \in \mathscr{O}$ there exist functions $\mathscr{F}_{n, k, n p}^{\varepsilon}, \mathscr{F}_{n, k, p}^{\varepsilon}, \mathscr{G}_{n, \ell, n p}^{\varepsilon}$ and $\mathscr{G}_{n, \ell, p}^{\varepsilon}$ such that we have the following decompositions

$$
\forall k \in \mathscr{I}, u_{n, k}^{\varepsilon}(t, x):=\mathscr{F}_{n, k, n p}^{\varepsilon}(t, x)+\mathscr{F}_{n, k, p}^{\varepsilon}(t, x)+\varrho_{n}^{\varepsilon}\left(\mathbf{t}_{\rightarrow}, \mathbf{x}_{\rightarrow}\right) e_{k}+\nu_{n}^{\varepsilon}\left(\mathbf{t}_{\rightarrow}, \mathbf{x}_{\rightarrow}\right) v_{n, *, k}
$$

and

$$
\begin{aligned}
\forall \ell \in \mathscr{O}, u_{n, \ell}^{\varepsilon}(t, x):= & \mathscr{G}_{n, \ell, n p}^{\varepsilon}(t, x)+\mathscr{G}_{n, \ell, p}^{\varepsilon}(t, x)-\sum_{k \in \mathscr{I}} e^{\frac{i}{\varepsilon}\left(\xi_{k}-\xi_{\ell}\right)} \varrho_{n}^{\varepsilon}\left(\mathbf{t}_{\leftarrow}, \mathbf{x}_{\leftarrow}\right) P^{\ell} \phi_{1} B_{1} e_{k} \\
& -\sum_{k \in \mathscr{I}} e^{\frac{i}{\varepsilon}\left(\xi_{k}-\xi_{\ell}\right)} \nu_{n}^{\varepsilon}\left(\mathbf{t}_{\leftarrow}, \mathbf{x}_{\leftarrow}\right) P^{\ell} \phi_{1} B_{1} v_{n, k, *} .
\end{aligned}
$$

Moreover $\mathscr{F}_{n, k, n p}^{\varepsilon}, \mathscr{F}_{n, k, p}^{\varepsilon}, \mathscr{G}_{n, \ell, n p}^{\varepsilon}$ and $\mathscr{G}_{n, \ell, p}^{\varepsilon}$ depend linearly on the $\varrho_{j}^{\varepsilon}$ and the $\nu_{j}^{\varepsilon}$ for $0 \leq j \leq n-1$. 
In the decomposition 59 (resp. 60 ), the terms $\mathscr{F}_{n, k, n p}^{\varepsilon}\left(\right.$ resp. $\mathscr{G}_{n, \ell, n p}^{\varepsilon}$ ) correspond to the unpolarized part of the amplitude $u_{n, k}^{\varepsilon}$ (resp. $\left.u_{n, \ell}^{\varepsilon}\right)$, whereas $\mathscr{F}_{n, k, p}^{\varepsilon}$ (resp. $\mathscr{G}_{n, \ell, p}^{\varepsilon}$ ) correspond to the polarized part of the amplitude $u_{n, k}^{\varepsilon}$ (resp. $\left.u_{n, \ell}^{\varepsilon}\right)$ that does not depend on the higher order unknowns $\varrho_{n+1}^{\varepsilon}$ and $\nu_{n+1}^{\varepsilon}$.

Proof : The proof is based on an iterative process.

Because of the results of Paragraph 4.3.1. The decompositions 59 and 60 (for $n=1$ ) holds with

$$
\begin{aligned}
\mathscr{F}_{1, k, n p}^{\varepsilon} & :=\left(I-P^{k}\right) u_{1, k}^{\varepsilon}=i\left(\mathcal{T} \varrho_{0}^{\varepsilon}\right)\left(\mathbf{t}_{\rightarrow}, \mathbf{x}_{\rightarrow}\right) R^{k} e_{k}, \\
\mathscr{F}_{1, k, p}^{\varepsilon} & :=-i \frac{x_{2}}{\mathbf{v}_{k, 2}}\left(\mathcal{T}^{2} \varrho_{0}^{\varepsilon}\right)\left(\mathbf{t}_{\rightarrow}, \mathbf{x}_{\rightarrow}\right) \widetilde{Q}^{k} Q^{k} R^{k} e_{k},
\end{aligned}
$$

and

$$
\begin{aligned}
\mathscr{G}_{1, \ell, n p}^{\varepsilon}:= & -i \sum_{k \in \mathscr{I}} e^{\frac{i}{\varepsilon}\left(\xi_{k}-\xi_{\ell}\right)}\left(\mathcal{T} \varrho_{0}^{\varepsilon}\right)\left(\mathbf{t}_{\leftarrow}, \mathbf{x}_{\leftarrow}\right) R^{\ell} P^{\ell} \phi_{1} B_{1} e_{k} \\
\mathscr{G}_{1, \ell, p}^{\varepsilon}:= & -i \sum_{k \in \mathscr{I}} e^{\frac{i}{\varepsilon}\left(\xi_{k}-\xi_{\ell}\right)}\left(\mathcal{T} \varrho_{0}^{\varepsilon}\right)\left(\mathbf{t}_{\leftarrow}, \mathbf{x}_{\leftarrow}\right) P^{\ell} \phi_{1} B_{1} R^{k} e_{k} \\
& +i \sum_{k \in \mathscr{I}} \frac{e^{\frac{i}{\varepsilon}\left(\xi_{k}-\xi_{\ell}\right)}}{\mathbf{v}_{k, 2}}\left(\mathcal{T}^{2} \varrho_{0}^{\varepsilon}\right)\left(\mathbf{t}_{\leftarrow}, \mathbf{x}_{\leftarrow}\right) P^{\ell} \phi_{1} B_{1} \widetilde{Q}^{k} Q^{k} R^{k} e_{k} \\
& +i \sum_{k \in \mathscr{I}} \sum_{\ell^{\prime} \in \mathscr{O}} e^{\frac{i}{\varepsilon}\left(\xi_{k}-\xi_{\ell}\right)}\left(\mathcal{T} \varrho_{0}^{\varepsilon}\right)\left(\mathbf{t}_{\leftarrow}, \mathbf{x}_{\leftarrow}\right) P^{\ell} \phi_{1} B_{1} R^{\ell^{\prime}} P^{\ell^{\prime}} \phi_{1} B_{1} e_{k} \\
& -i \frac{\left(1-x_{2}\right)}{\mathbf{v}_{\ell, 2}} \sum_{k \in \mathscr{I}} e^{\frac{i}{\varepsilon}\left(\xi_{k}-\xi_{\ell}\right)}\left(\mathcal{T}^{2} \varrho_{0}^{\varepsilon}\right)\left(\mathbf{t}_{\leftarrow}, \mathbf{x}_{\leftarrow}\right) \widetilde{Q}^{\ell} Q^{\ell} R^{\ell} P^{\ell} \phi_{1} B_{1} e_{k} .
\end{aligned}
$$

Assume that $(59)$ and $(60)$ hold at the order $n$. Then following the computations performed in Paragraph 4.3.1 and using the standard relation for unpolarized parts we define for all $k \in \mathscr{I}$

$$
\mathscr{F}_{n+1, k, n p}^{\varepsilon}(t, x):=i R^{k} L(\partial)\left(\mathscr{F}_{n, k, n p}^{\varepsilon}+\mathscr{F}_{n, k, p}^{\varepsilon}\right)(t, x)+i\left(\mathcal{T} \varrho_{n}^{\varepsilon}\right)\left(\mathbf{t}_{\rightarrow}, \mathbf{x}_{\rightarrow}\right) R^{k} e_{k}+i\left(\mathcal{T}_{\nu_{n}^{\varepsilon}}^{\varepsilon}\right)\left(\mathbf{t}_{\rightarrow}, \mathbf{x}_{\rightarrow}\right) R^{k} v_{n, k, *}
$$

and for all $\ell \in \mathscr{O}$

$$
\begin{aligned}
\mathscr{G}_{n+1, \ell, n p}^{\varepsilon}(t, x):= & i R^{\ell} L(\partial)\left(\mathscr{G}_{n, \ell, n p}^{\varepsilon}+\mathscr{G}_{n, \ell, p}^{\varepsilon}\right)(t, x)-i \sum_{k \in \mathscr{I}} e^{\frac{i}{\varepsilon}\left(\xi_{k}-\xi_{\ell}\right)}\left(\mathcal{T} \varrho_{n}^{\varepsilon}\right)\left(\mathbf{t}_{\leftarrow}, \mathbf{x}_{\leftarrow}\right) R^{\ell} P^{\ell} \phi_{1} B_{1} e_{k} \\
& -i \sum_{k \in \mathscr{I}} e^{\frac{i}{\varepsilon}\left(\xi_{k}-\xi_{\ell}\right)}\left(\mathcal{T} \nu_{n}^{\varepsilon}\right)\left(\mathbf{t}_{\leftarrow}, \mathbf{x}_{\leftarrow}\right) R^{\ell} P^{\ell} \phi_{1} B_{1} v_{n, k, *} .
\end{aligned}
$$

In particular, it is clear from the iterative assumption, that such $\mathscr{F}_{n+1, k, n p}^{\varepsilon}$ and $\mathscr{G}_{n+1, \ell, n p}^{\varepsilon}$ depend linearly on the $\varrho_{j}^{\varepsilon}$ and the $\nu_{j}^{\varepsilon}$ for $0 \leq j \leq n$. So that to determine the terms $\mathscr{F}_{n+1, k, p}^{\varepsilon, h, n p}$ and $\mathscr{G}_{n+1, \ell, p}^{\varepsilon}$ we consider the polarized parts $P^{k} u_{n+1, k}^{\varepsilon}$ and $P^{\ell} u_{n+1, \ell}^{\varepsilon}$. Once again reiterating essentially the computations performed in Paragraph 4.3.1 we have to solve the transport equations:

$$
\forall k \in \mathscr{I},\left\{\begin{array}{l}
\left(\partial_{t}+\mathbf{v}_{k} \cdot \nabla_{x}\right) P^{k} u_{n+1, k}^{\varepsilon}=-\widetilde{Q}^{k} Q^{k} L(\partial) \mathscr{F}_{n, k, n p}^{\varepsilon} \\
P^{k} u_{n+1, k_{\mid x_{2}=0}^{\varepsilon}}^{\varepsilon}=\varrho_{n, k}^{\varepsilon} e_{k}+\nu_{n+1}^{\varepsilon} v_{n+1, k, *} \\
u_{n+1, k}^{\varepsilon} \in \mathscr{P}_{n+1}
\end{array}\right.
$$

and

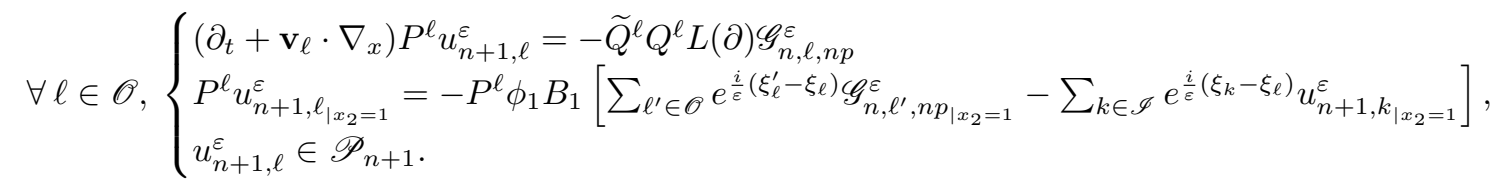


Integrating (63) along the characteristics implies that the decomposition 59 holds at the order $n+1$ if we define:

$$
\begin{aligned}
& \mathscr{F}_{n+1, k, p}^{\varepsilon}(t, x)=-i \frac{x_{2}}{\mathbf{v}_{k, 2}} \widetilde{Q}^{k} Q^{k}\left[L(\partial) R^{k} L(\partial)\left(\mathscr{F}_{n, k, n p}^{\varepsilon}+\mathscr{F}_{n, k, p}^{\varepsilon}\right)(t, x)\right. \\
& \left.+\left(\left(\mathscr{T}^{2} \varrho_{n}^{\varepsilon}\right) R^{k} e_{k}+\left(\mathscr{T}^{2} \nu_{n}^{\varepsilon}\right) R^{k} v_{n, k, *}\right)\left(t-\frac{1}{\mathbf{v}_{k, 2}} x_{2}, x_{1}-\frac{\mathbf{v}_{k, 1}}{\mathbf{v}_{k, 2}} x_{2}\right)\right],
\end{aligned}
$$

which clearly depends linearly on the $\varrho_{j}^{\varepsilon}$ and the $\nu_{j}^{\varepsilon}$ for $0 \leq j \leq n$.

Using this expression in the transport equation (64) then shows that the decomposition (59) holds at the order $n+1$ if we define:

$$
\begin{aligned}
\mathscr{G}_{n+1, \ell, p}^{\varepsilon}(t, x)= & \frac{1-x_{2}}{\mathbf{v}_{\ell, 2}} \widetilde{Q}^{\ell} Q^{\ell}\left(L(\partial) \mathscr{G}_{n+1, \ell, n p}^{\varepsilon}\right)(t, x) \\
& -P^{\ell} \phi_{1} B_{1} \sum_{\ell^{\prime} \in \mathscr{O}} e^{\frac{i}{\varepsilon}\left(\xi_{\ell^{\prime}}-\xi_{\ell}\right)} \mathscr{G}_{n+1, k, n p_{\mid x_{2}=1}^{\varepsilon}}^{\varepsilon}\left(t+\frac{1-x_{2}}{\mathbf{v}_{\ell, 2}}, x_{1}+\left(1-x_{2}\right) \frac{\mathbf{v}_{\ell, 1}}{\mathbf{v}_{\ell, 2}}\right) \\
& -P^{\ell} \phi_{1} B_{1} \sum_{k \in \mathscr{I}} e^{\frac{i}{\varepsilon}\left(\xi_{k}-\xi_{\ell}\right)}\left[\mathscr{F}_{n+1, k, n p_{\mid x_{2}=1}}^{\varepsilon}+\mathscr{F}_{n+1, k, p_{\mid x_{2}=1}}^{\varepsilon}\right]\left(t+\frac{1-x_{2}}{\mathbf{v}_{\ell, 2}}, x_{1}+\left(1-x_{2}\right) \frac{\mathbf{v}_{\ell, 1}}{\mathbf{v}_{\ell, 2}}\right) .
\end{aligned}
$$

We also have the proposition

Proposition 4.2 For all $n \geq 1$ we have the following

$$
\begin{aligned}
& \forall k \in \mathscr{I}, \mathscr{F}_{n, k, n p_{\mid x_{2}=0}=0}^{\varepsilon}, \mathscr{F}_{n, k, n p_{\mid x_{2}=1}^{\varepsilon}}^{\varepsilon} \in \mathscr{P}_{n-1} \text { and } \mathscr{F}_{n, k, n p_{\mid x_{2}=0}^{\varepsilon}}^{\varepsilon} \equiv 0, \mathscr{F}_{n, k, n p_{\mid x_{2}=1}}^{\varepsilon} \in \mathscr{P}_{n-1} \\
& \forall \ell \in \mathscr{O}, \mathscr{G}_{n, \ell,\left.n\right|_{\mid x_{2}=1} ^{\varepsilon}}^{\varepsilon}, \mathscr{G}_{n, \ell, p_{\mid x_{2}=1}}^{\varepsilon} \in \mathscr{P}_{n-1} \text { and } \mathscr{G}_{n, \ell,\left.n\right|_{\mid x_{2}=0} ^{\varepsilon}}, \mathscr{G}_{n, \ell, p_{\mid x_{2}=0}}^{\varepsilon} \in \mathscr{P}_{n-2} .
\end{aligned}
$$

Proof : Once again the proof relies on an iterative argument. Clearly from the initialization made in the proof of Proposition 4.1, Proposition 4.2 holds for $n=1$.

Assume that Proposition 4.2 holds for some $n$ then from (61) combined with $\mathbf{t}_{\rightarrow_{\mid x_{2}=0}}=t$ and because $L(\partial)$ and $\mathcal{T}$ leave the space $\mathscr{P}_{n}$ invariant, it is clear that $\mathscr{F}_{n+1, k, n p_{\mid x_{2}=0}^{\varepsilon}}^{\varepsilon} \in \mathscr{P}_{n}$. Indeed the first term in the right hand side of (61) is in $\mathscr{P}_{n-1}$ by the iterative assumption, the second one is in $\mathscr{P}_{n}$ by definition of $\varrho_{n}^{\varepsilon}$ and the last one lies in $\mathscr{P}_{n-1}$ by definition of $\nu_{n}^{\varepsilon}$.

Because $\mathbf{t}_{\rightarrow_{\mid x_{2}=1}}=t-\frac{1}{\mathbf{v}_{k, 2}}$, it is also clear from 61] and 65 that $\mathscr{F}_{n, k,\left.n\right|_{\mid x_{2}=1} ^{\varepsilon}}, \mathscr{F}_{n, k, p_{\mid x_{2}=1}^{\varepsilon}}^{\varepsilon} \in \mathscr{P}_{n}$.

Concerning the terms $\mathscr{G}_{n+1, \ell, p}^{\varepsilon}$ and $\mathscr{G}_{n+1, \ell, n p}^{\varepsilon}$ the iterative assumption combined with the equality $\mathbf{t}_{\leftarrow \mid x_{2}=1}=$ $\mathbf{t}_{\rightarrow \mid x_{2}=1}$ implies (using (62) and (66))that $\mathscr{G}_{n+1, \ell, p}^{\varepsilon}, \mathscr{G}_{n+1, \ell, n p}^{\varepsilon} \in \mathscr{P}_{n}$ (the limiting term in $\mathscr{G}_{n+1, \ell, n p}^{\varepsilon}$ is $\left(\mathcal{T} \varrho_{n}^{\varepsilon}\right)\left(\mathbf{t}_{\leftarrow \mid x_{2}=1}, \cdot\right)$ with a repercussion in 66$)$.

Finally the iterative assumption and the equality $\mathbf{t}_{\leftarrow \mid x_{2}=0}=\alpha_{k, \ell}$ shows that the first in the right hand side of $(62)$ is in $\mathscr{P}_{n-2}$, the second one in $\mathscr{P}_{n-1}$ and the last one in $\mathscr{P}_{n-2}$. Consequently $\mathscr{G}_{n+1, \ell, n p}^{\varepsilon} \in \mathscr{P}_{n-1}$.

Using this result in the expression of $\mathscr{G}_{n+1, \ell, p}^{\varepsilon}$ shows that the limiting term in the right hand side of 66. is the first one (which lies in $\mathscr{P}_{n-1}$, the other ones are in $\mathscr{P}_{n-2}$ ).

First reformulation : $\nu_{n}^{\varepsilon}$ as a function of the $\varrho_{j}^{\varepsilon}$ for $j \in \llbracket 0, n \rrbracket$ The aim of this paragraph is to show that the (unknown) "almost-stable" component of the trace of the incoming modes on $\partial \Omega_{0, T}$ can be expressed in terms of the "purely-unstable" components that is the $\varrho_{j}^{\varepsilon}$ for all $j \in \llbracket 0, n \rrbracket$.

The first step is to consider the boundary condition (23) at the order $n$. Multiplying this equation by $\phi_{0, *}$ in the left hand side we obtain that

$$
\forall k \in \mathscr{I}, \nu_{n}^{\varepsilon} v_{n, k, *}=\delta_{n+1, K} P^{k} \phi_{0, *} g-P^{k} \phi_{0, *} B_{0} \sum_{k^{\prime} \in \mathscr{I}}\left(I-P^{k^{\prime}}\right) u_{n, k_{\mid x_{2}=0}^{\prime}}^{\varepsilon}-P^{k} \phi_{0, *} B_{0} \sum_{\ell \in \mathscr{O}} u_{n, \ell_{\mid x_{2}=0}^{\varepsilon}},
$$


equation that from Proposition 4.1 reads:

$$
\begin{aligned}
\left(I-\mathcal{S}_{*}^{\varepsilon}\right) \nu_{n}^{\varepsilon} v_{n, *}= & \delta_{n, K+1} P^{k} \phi_{0, *} g+\mathcal{S}_{*}^{\varepsilon} \varrho_{n}^{\varepsilon}-\phi_{0, *} B_{0} \sum_{k \in \mathscr{I}} \mathscr{F}_{n, k, n p_{\mid x_{2}=0}}^{\varepsilon} \\
& -\phi_{0, *} B_{0} \sum_{\ell \in \mathscr{O}} \mathscr{G}_{n, \ell, n p_{\mid x_{2}=0}^{\varepsilon}}^{\varepsilon}-\phi_{0, *} B_{0} \sum_{\ell \in \mathscr{O}} \mathscr{G}_{n, \ell, p_{\mid x_{2}=0}}^{\varepsilon} .
\end{aligned}
$$

From Propositions 4.1 and 4.2 we can write?

$$
\sum_{k \in \mathscr{I}} \mathscr{F}_{n, k, n p_{\mid x_{2}=0}}^{\varepsilon}:=\sum_{m=0}^{n-1} \mathbb{F}_{n, m}^{\varepsilon}+\sum_{m=0}^{n-1} \mathbb{F}_{n, m, *}^{\varepsilon} \text { and } \sum_{\ell \in \mathscr{O}} \mathscr{G}_{n, \ell, n p_{\mid x_{2}=0}^{\varepsilon}}+\mathscr{G}_{n, \ell, p_{\mid x_{2}=0}}^{\varepsilon}:=\sum_{j=0}^{n-1} \mathbb{G}_{n, m}^{\varepsilon}+\sum_{j=0}^{n-1} \mathbb{G}_{n, m, *}^{\varepsilon},
$$

where (with a slight abuse of notations) for all $n \geq 0, m \in \llbracket 0, n-1 \rrbracket, \mathbb{F}_{n, m}^{\varepsilon}$ and $\mathbb{G}_{n, m}^{\varepsilon}$ (resp. $\mathbb{F}_{n, m, *}^{\varepsilon}$ and $\left.\mathbb{G}_{n, m, *}^{\varepsilon}\right)$ are linear operators that only depend on $\varrho_{m}^{\varepsilon}$ (resp. $\left.\nu_{m}^{\varepsilon}\right)$ and satisfying that $\mathbb{F}_{n, m}^{\varepsilon, m}, \mathbb{G}_{n, m}^{\varepsilon} \in \mathscr{P}_{m}$ (resp. $\left.\mathbb{F}_{n, m, *}^{\varepsilon}, \mathbb{G}_{n, m, *}^{\varepsilon} \in \mathscr{P}_{m-1}\right)$.

With these new notations in hand, from the Neumann series expansion of $I-\mathcal{S}_{*}^{\varepsilon}$, 67) becomes for all $\mathrm{n}$ $\in \mathbb{N}$,

$$
\nu_{n}^{\varepsilon} v_{n, *}=\delta_{n, K+1} \sum_{j=0}^{K}\left(\mathcal{S}_{*}^{\varepsilon}\right)^{j} \phi_{0, *} g+\sum_{m=0}^{n-1} \sum_{j=0}^{\min (m, K)}\left(\mathcal{S}_{*}^{\varepsilon}\right)^{j} \widetilde{\mathscr{V}}_{n, m}^{\varepsilon}+\sum_{m=1}^{n-1} \sum_{j=0}^{\min (m-1, K)}\left(\mathcal{S}_{*}^{\varepsilon}\right)^{j} \widetilde{\mathscr{V}}_{n, m, *}^{\varepsilon}+\sum_{j=1}^{\min (n, K)}\left(\mathcal{S}_{*}^{\varepsilon}\right)^{j} \varrho_{n}^{\varepsilon} e,
$$

where we defined

$$
\widetilde{\mathscr{V}}_{n, m}^{\varepsilon}=-\phi_{0, *} B_{0}\left(\mathbb{F}_{n, m}^{\varepsilon}+\mathbb{G}_{n, m}^{\varepsilon}\right) \in \mathscr{P}_{m} \text { and } \widetilde{\mathscr{V}}_{n, m}:=-\phi_{0, *} B_{0}\left(\mathbb{F}_{n, m, *}^{\varepsilon}+\mathbb{G}_{n, m, *}^{\varepsilon}\right) \in \mathscr{P}_{m-1} .
$$

Equation (69) essentially gives the expression of $\nu_{n}^{\varepsilon}$ in terms of the $\varrho_{m}^{\varepsilon}, \nu_{q}^{\varepsilon}$ for $m \in \llbracket 0, n \rrbracket, q \in \llbracket 1, n-1 \rrbracket$.

The main result of this paragraph is then to show that up to change the definition of the $\widetilde{\mathcal{V}}_{n, m}^{\varepsilon}, \widetilde{\mathscr{V}}_{n, m, *}^{\varepsilon}$ we can restrict the second sum in the right hand side of 699 to $m \in \llbracket 1, n-2 \rrbracket$. In other words we can express $\nu_{n}^{\varepsilon}$ in terms of the $\varrho_{m}^{\varepsilon}$ for $m \in \llbracket 0, n-1 \rrbracket$ and the $\nu_{q}^{\varepsilon}$ for $q \in \llbracket 0, n-2 \rrbracket$. Consequently an iterative argument (recall that $\nu_{0}^{\varepsilon} \equiv 0$ ) shows that for all $n \in \mathbb{N}$ we can express $\nu_{n}^{\varepsilon}$ in terms of the $\varrho_{m}^{\varepsilon}$ for $m \in \llbracket 0, n-1 \rrbracket$ only.

We give here the details of the computations for the special case $n=K+1$. Firstly because this term will be of particular importance in the rest of the proof (more specifically in the reformulation of the equation determining $\varrho_{n+1}^{\varepsilon}$ in terms of $\varrho_{n}^{\varepsilon}$ ) and secondly because this term is the most delicate to handle with because of the source term $g$.

Proposition 4.3 For all $m \in \llbracket 0, K \rrbracket$ (resp. $m \in \llbracket 1, K-1 \rrbracket$ ), there exist linear operators $\mathscr{V}_{K+1, m}^{\varepsilon}$ (resp. $\left.\mathscr{V}_{K+1, m, *}^{\varepsilon}\right)$ only depending on $\varrho_{m}^{\varepsilon}\left(\right.$ resp. $\left.\nu_{m}^{\varepsilon}\right)$ such that

$$
\nu_{K+1}^{\varepsilon} v_{K+1, *}=\sum_{j=0}^{K}\left(\mathcal{S}_{*}^{\varepsilon}\right)^{j} \phi_{0, *} g+\sum_{m=0}^{K} \sum_{j=0}^{m}\left(\mathcal{S}_{*}^{\varepsilon}\right)^{j} \mathscr{V}_{K+1, m}^{\varepsilon}+\sum_{m=1}^{K-1} \sum_{j=0}^{m-1}\left(\mathcal{S}_{*}^{\varepsilon}\right)^{j} \mathscr{V}_{K+1, m, *}^{\varepsilon}+\sum_{j=1}^{K}\left(\mathcal{S}_{*}^{\varepsilon}\right)^{j} \varrho_{K+1}^{\varepsilon} e
$$

Proof : We start from equation 69 written for $n=K+1$ in which we isolate the only term depending on $\nu_{K}^{\varepsilon}\left(\right.$ namely $\left.\widetilde{\mathscr{V}}_{K+1, K, *}^{\varepsilon}\right)$ :

$$
\begin{aligned}
\nu_{K+1}^{\varepsilon} v_{K+1, *}= & \sum_{j=0}^{K}\left(\mathcal{S}_{*}^{\varepsilon}\right)^{j} \phi_{0, *} g+\sum_{m=0}^{K} \sum_{j=0}^{m}\left(\mathcal{S}_{*}^{\varepsilon}\right)^{j} \widetilde{\mathscr{V}}_{K+1, m}^{\varepsilon}+\sum_{m=1}^{K-1} \sum_{j=0}^{m-1}\left(\mathcal{S}_{*}^{\varepsilon}\right)^{j} \widetilde{\mathscr{V}}_{K+1, m, *}^{\varepsilon}+\sum_{j=1}^{K}\left(\mathcal{S}_{*}^{\varepsilon}\right)^{j} \varrho_{K+1}^{\varepsilon} e \\
& +\sum_{j=0}^{K-1}\left(\mathcal{S}_{*}^{\varepsilon}\right)^{j} \widetilde{\mathscr{V}}_{K+1, K, *}^{\varepsilon}\left(\nu_{K}^{\varepsilon} v_{K, *}\right) .
\end{aligned}
$$

\footnotetext{
${ }^{9}$ As the reader will notice, the precise expression of the operators $\mathbb{F}_{n, m}^{\varepsilon}, \mathbb{F}_{n, m, *}^{\varepsilon}$ and $\mathbb{G}_{n, m}^{\varepsilon}, \mathbb{G}_{n, m, *}^{\varepsilon}$ is of little interest for the following. Indeed the only interesting point is their behaviour compared to the profile spaces. So to save some tedious formula we will not give the precise expressions here.
} 
From equation 69 written for $n=K$ we also have

$$
\nu_{K}^{\varepsilon} v_{K, *}=\sum_{m=0}^{K-1} \sum_{p=0}^{m}\left(\mathcal{S}_{*}^{\varepsilon}\right)^{p} \widetilde{\mathscr{V}}_{K, m}^{\varepsilon}+\sum_{m=1}^{K-1} \sum_{p=0}^{m-1}\left(\mathcal{S}_{*}^{\varepsilon}\right)^{p} \widetilde{\mathscr{V}}_{K, m, *}^{\varepsilon}+\sum_{p=1}^{K}\left(\mathcal{S}_{*}^{\varepsilon}\right)^{p} \varrho_{K}^{\varepsilon} e,
$$

from which it follows (recall that $\widetilde{\mathscr{V}}_{K+1, K, *}^{\varepsilon}$ is linear with respect to $\nu_{K}^{\varepsilon} v_{K, *}$ ) that

$$
\begin{aligned}
\sum_{j=0}^{K-1}\left(\mathcal{S}_{*}^{\varepsilon}\right)^{j} \widetilde{\mathscr{V}}_{K+1, K, *}^{\varepsilon}\left[\nu_{K}^{\varepsilon} v_{K, *}\right]= & \sum_{m=0}^{K-1} \sum_{j=0}^{K-1}\left(\mathcal{S}_{*}^{\varepsilon}\right)^{j} \underbrace{\sum_{p=0}^{m} \widetilde{\mathscr{V}}_{K+1, K, *}^{\varepsilon}\left[\left(\mathcal{S}_{*}^{\varepsilon}\right)^{p} \widetilde{\mathscr{V}}_{K, m}^{\varepsilon}\right]}_{:=\widetilde{\mathscr{V}}_{K+1, m}^{\varepsilon}} \\
& +\sum_{m=1}^{K-1} \sum_{j=0}^{K-1}\left(\mathcal{S}_{*}^{\varepsilon}\right)^{j} \underbrace{}_{:=\widetilde{\mathscr{V}}_{K+1, m}^{\varepsilon} \underbrace{m-1}_{j=0} \widetilde{\mathscr{V}}_{K+1, K, *}^{\varepsilon}\left[\left(\mathcal{S}_{*}^{\varepsilon}\right)^{p} \widetilde{\mathscr{V}}_{K, m, *}^{\varepsilon}\right]} \\
& +\sum_{j=0}^{K-1} \sum_{p=1}^{K}\left(\mathcal{S}_{*}^{\varepsilon}\right)^{j} \widetilde{\mathscr{V}}_{K+1, K, *}^{\varepsilon}\left[\left(\mathcal{S}_{*}^{\varepsilon}\right)^{p} \varrho_{K}^{\varepsilon} e\right] .
\end{aligned}
$$

For all $m \in \llbracket 0, K-1 \rrbracket, p \in \llbracket 0, m \rrbracket$, the $\widetilde{\mathscr{V}}_{K+1, K, *}^{\varepsilon}\left[\left(\mathcal{S}_{*}^{\varepsilon}\right)^{p} \widetilde{\mathscr{V}}_{K, m}^{\varepsilon}\right] \in \mathscr{P}_{m-p} \subset \mathscr{P}_{m}$ and only depend on the

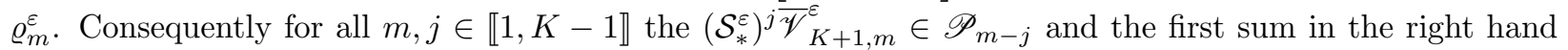
side of 73 can be restricted to $j \in \llbracket 0, m \rrbracket$.

Similarly for all $m \in \llbracket 0, K-1 \rrbracket, p \in \llbracket 0, m-1 \rrbracket$, the $\widetilde{\mathscr{V}}_{K+1, K, *}^{\varepsilon}\left[\left(\mathcal{S}_{*}^{\varepsilon}\right)^{p} \widetilde{\mathscr{V}}_{K, m}^{\varepsilon}\right] \in \mathscr{P}_{m-1-p} \subset \mathscr{P}_{m}$ so the second sum in the right hand side of $(73)$ can be restricted to $j \in \llbracket 0, m-1 \rrbracket$.

Using these remarks in (71) shows that (70) holds if we define:

$$
\begin{aligned}
& \forall m \in \llbracket 1, K-1 \rrbracket, \mathscr{V}_{K+1, m, *}^{\varepsilon}=\widetilde{\mathscr{V}}_{K+1, m, *}^{\varepsilon}+\overline{\mathscr{V}}_{K+1, m, *}^{\varepsilon} \in \mathscr{P}_{m-1}, \\
& \forall m \in \llbracket 0, K-1 \rrbracket, \mathscr{V}_{K+1, m}^{\varepsilon}=\widetilde{\mathscr{V}}_{K+1, m}^{\varepsilon}+\overline{\mathscr{V}}_{K+1, m}^{\varepsilon} \in \mathscr{P}_{m} \\
& \text { and } \mathscr{V}_{K+1, K}^{\varepsilon}=\widetilde{\mathscr{V}}_{K+1, K}^{\varepsilon}+\sum_{p=1}^{K} \widetilde{\mathscr{V}}_{K+1, K, *}^{\varepsilon}\left[\left(\mathcal{S}_{*}^{\varepsilon}\right)^{p} \varrho_{K}^{\varepsilon} e\right] \in \mathscr{P}_{K}
\end{aligned}
$$

With Proposition 4.3 in hand, by an iterative argument, we can state the main result of this paragraph. Namely that the $\nu_{n}^{\varepsilon}$ can be expressed in terms of the $\varrho_{m}^{\varepsilon}$ for $m \in \llbracket 0, n+1 \rrbracket$, more precisely

Proposition 4.4 For all $n \geq 1, m \in \llbracket 0, K \rrbracket$, there exist linear operators $\mathscr{W}_{n, m}^{\varepsilon}$ only depending on $\varrho_{m}^{\varepsilon}$ and satisfying $\mathscr{W}_{n, m}^{\varepsilon} \in \mathscr{P}_{m}$ such that

$$
\nu_{n}^{\varepsilon} v_{n, *}=\mathscr{G}_{n} g+\sum_{m=0}^{n-1} \sum_{j=0}^{m}\left(\mathcal{S}_{*}^{\varepsilon}\right)^{j} \mathscr{W}_{n, m}^{\varepsilon}+\sum_{j=1}^{\min (n, K)}\left(\mathcal{S}_{*}^{\varepsilon}\right)^{j} \varrho_{n}^{\varepsilon} e,
$$

where for all $n \geq 0, \mathscr{G}_{n}$ is a linear operator (only depending on the source term) such that: $\mathscr{G}_{n}=0$ if $n \leq K$ and $\mathscr{G}_{K+1}:=\sum_{j=0}^{K}\left(\mathcal{S}_{*}^{\varepsilon}\right)^{j} \phi_{0, *}$.

Second reformulation: $\varrho_{n}^{\varepsilon}$ as a function of the $\varrho_{m}^{\varepsilon}, m \in \llbracket 0, n-1 \rrbracket$. The aim of this paragraph is to give a relation expressing $\varrho_{n+1}^{\varepsilon}$ in terms of the $\varrho_{m}^{\varepsilon}$, for $m \in \llbracket 0, n \rrbracket$. 
Taking the scalar product of the boundary condition (23) at the order $n+1$ by $b$ (see Definition 4.2 ) reads, thanks to Proposition 4.1 .

$$
\begin{aligned}
b \cdot B_{0} \sum_{k \in \mathscr{I}} \mathscr{F}_{n+1, k, n p_{\mid x_{2}=0}^{\varepsilon}=} & \delta_{n, K} b \cdot g-\sum_{\ell \in \mathscr{O}} \mathscr{G}_{n+1, \ell, n p_{\mid x_{2}=0}^{\varepsilon}}+\mathscr{G}_{n+1, \ell, p_{\mid x_{2}=0}^{\varepsilon}} \\
& +b \cdot B_{0} \sum_{k \in \mathscr{I}} \sum_{\ell \in \mathscr{O}} e^{\frac{i}{\varepsilon}\left(\xi_{k}-\xi_{\ell}\right)} \varrho_{n+1}^{\varepsilon}\left(t-\alpha_{k, \ell}, x_{1}-\beta_{k, \ell}\right) P^{\ell} \phi_{1} B_{1} e_{k} \\
& +b \cdot B_{0} \sum_{k \in \mathscr{I}} \sum_{\ell \in \mathscr{O}} e^{\frac{i}{\varepsilon}\left(\xi_{k}-\xi_{\ell}\right)} \nu_{n+1}^{\varepsilon}\left(t-\alpha_{k, \ell}, x_{1}-\beta_{k, \ell}\right) P^{\ell} \phi_{1} B_{1} v_{n+1, k, *} .
\end{aligned}
$$

In the spirit of the previous paragraph, in order to fix the ideas and save some notations, we describe in this paragraph the computations for $n=K$. From 68 we thus have:

$$
\begin{aligned}
b \cdot B_{0}\left(\sum_{m=0}^{K} \mathbb{F}_{K+1, m}^{\varepsilon}+\sum_{m=1}^{K} \mathbb{F}_{K+1, m, *}^{\varepsilon}\right)= & b \cdot g-b \cdot B_{0}\left(\sum_{m=0}^{K} \mathbb{G}_{K+1, m}^{\varepsilon}+\sum_{m=1}^{K} \mathbb{G}_{K+1, m, *}^{\varepsilon}\right) \\
& +\widetilde{\mathcal{R}}^{\varepsilon}\left(\varrho_{K+1}^{\varepsilon} e\right)+\widetilde{\mathcal{R}}^{\varepsilon}\left(\nu_{K+1}^{\varepsilon} v_{K+1, *}\right),
\end{aligned}
$$

where we defined for a scalar function $f$ defined on $\partial \Omega_{0, T}$ and $v \in \mathbb{C}^{N}$ we defined

$$
\widetilde{\mathcal{R}}^{\varepsilon}(f v):=b \cdot B_{0} \sum_{k \in \mathscr{I}} \sum_{\ell \in \mathscr{O}} e^{\frac{i}{\varepsilon}\left(\xi_{k}-\xi_{\ell}\right)} f\left(t-\alpha_{k, \ell}, x_{1}-\beta_{k, \ell}\right) P^{\ell} \phi_{1} B_{1} P^{k} v .
$$

The aim of the end of the paragraph is to rewrite $(76)$ under the generic form

$$
(I-\mathscr{H})\left(\varrho_{K}^{\varepsilon}\right)=\mathscr{F}_{-1}(g)+\sum_{m=0}^{K-1} \mathscr{F}_{m}\left(\varrho_{m}^{\varepsilon}\right)+\mathscr{F}_{K+1}\left(\varrho_{K+1}^{\varepsilon}\right),
$$

where the $\mathscr{F}_{j}$ are some linear operators to be determined and where $\mathscr{H}$ is a linear operator "costing at least the time needed to perform a full regeneration". As a consequence, the Neumann series expansion of $I-\mathscr{H}$, contains a finite number of terms $\varrho_{K}^{\varepsilon}$

In order to do so, we make 76 more explicit in terms $\varrho_{K}^{\varepsilon}$. In the following we list all the terms involving

- Obvious terms. Because of 68 , the terms $\mathbb{F}_{K+1, K}^{\varepsilon}$ and $\mathbb{G}_{K+1, K}^{\varepsilon}$ in 76 clearly involve $\varrho_{K}^{\varepsilon}$. Moreover from 65 we can write:

$$
\begin{aligned}
b \cdot B_{0} \sum_{m=0}^{K} \mathbb{F}_{K+1, m}^{\varepsilon} & =b \cdot B_{0} \sum_{m=0}^{K-1} \mathbb{F}_{K+1, m}^{\varepsilon}+b \cdot B_{0} \mathbb{F}_{K+1, K}^{\varepsilon} \\
& =b \cdot B_{0} \sum_{m=0}^{K-1} \mathbb{F}_{K+1, m}^{\varepsilon}+i b \cdot B_{0} \sum_{k \in \mathscr{I}}\left(\mathcal{T} \varrho_{K}^{\varepsilon}\right) R^{k} P^{k} e
\end{aligned}
$$


and from 62 and 66 some computations show that we can write:

$$
\begin{aligned}
b \cdot B_{0} \sum_{m=0}^{K} \mathbb{G}_{K+1, m}^{\varepsilon}= & \cdot B_{0} \sum_{m=0}^{K-1} \mathbb{G}_{K+1, m}^{\varepsilon} \\
& -i b \cdot B_{0} \sum_{k \in \mathscr{I}} \sum_{\ell \in \mathscr{O}} e^{\frac{i}{\varepsilon}\left(\xi_{k}-\xi_{\ell}\right)}\left(\mathcal{T} \varrho_{K}^{\varepsilon}\right)\left(t-\alpha_{k, \ell}, x_{1}-\beta_{k, \ell}\right) R^{\ell} P^{\ell} \phi_{1} B_{1} P^{k} e \\
& -i b \cdot B_{0} \sum_{k \in \mathscr{I}} \sum_{\ell \in \mathscr{O}} e^{\frac{i}{\varepsilon}\left(\xi_{k}-\xi_{\ell}\right)}\left(\mathcal{T}_{\varrho_{K}^{\varepsilon}}^{\varepsilon}\right)\left(t-\alpha_{k, \ell}, x_{1}-\beta_{k, \ell}\right) P^{\ell} \phi_{1} B_{1} R^{k} P^{k} e \\
& +i b \cdot B_{0} \sum_{k \in \mathscr{I}} \sum_{\ell \in \mathscr{O}} \frac{e^{\frac{i}{\varepsilon}\left(\xi_{k}-\xi_{\ell}\right)}}{\mathbf{v}_{k, 2}}\left(\mathcal{T}^{2} \varrho_{K}^{\varepsilon}\right)\left(t-\alpha_{k, \ell}, x_{1}-\beta_{k, \ell}\right) P^{\ell} \phi_{1} B_{1} \widetilde{Q}^{k} Q^{k} R^{k} P^{k} e \\
& -i b \cdot B_{0} \sum_{k \in \mathscr{I}} \sum_{\ell \in \mathscr{O}} \frac{e^{\frac{i}{\varepsilon}\left(\xi_{k}-\xi_{\ell}\right)}}{\mathbf{v}_{\ell, 2}}\left(\mathcal{T}^{2} \varrho_{K}^{\varepsilon}\right)\left(t-\alpha_{k, \ell}, x_{1}-\beta_{k, \ell}\right) \widetilde{Q}^{\ell} Q^{\ell} R^{\ell} P^{\ell} \phi_{1} B_{1} P^{k} e \\
& +i b \cdot B_{0} \sum_{k \in \mathscr{I}} \sum_{\ell \in \mathscr{O}} \sum_{\ell^{\prime} \in \mathscr{O}} e^{\frac{i}{\varepsilon}\left(\xi_{k}-\xi_{\ell}\right)}\left(\mathcal{T} \varrho_{K}^{\varepsilon}\right)\left(t-\alpha_{k, \ell}, x_{1}-\beta_{k, \ell}\right) P^{\ell} \phi_{1} B_{1} P^{\ell^{\prime}} \phi_{1} B_{1} R^{k} P^{k} e .
\end{aligned}
$$

So that at this step of the discussion using (78) and $(79)$ we write $(76)$ as follows:

$$
\begin{aligned}
i\left(\mathcal{C}-\widetilde{\mathcal{H}}^{\varepsilon}\right)\left(\varrho_{K}^{\varepsilon} e\right)+b \cdot B_{0} \sum_{m=1}^{K} \mathbb{F}_{K+1, m, *}^{\varepsilon}+b \cdot B_{0} \sum_{m=1}^{K} \mathbb{G}_{K+1, m, *}^{\varepsilon}= & b \cdot g-b \cdot B_{0} \sum_{m=0}^{K-1} \mathbb{F}_{K+1, m}^{\varepsilon} \\
& -b \cdot B_{0} \sum_{m=0}^{K-1} \mathbb{G}_{K+1, m}^{\varepsilon} \\
& +\widetilde{\mathcal{R}}^{\varepsilon}\left(\varrho_{K+1}^{\varepsilon} e\right)+\widetilde{\mathcal{R}}^{\varepsilon}\left(\nu_{K+1}^{\varepsilon} v_{K+1, *}\right)
\end{aligned}
$$

where for $v \in \mathbb{C}^{N}$ and $f$ a scalar function the operators $\mathcal{C}: \mathbb{C}^{N} \rightarrow \mathbb{C}$ and $\widetilde{\mathcal{H}}^{\varepsilon}: \mathbb{C}^{N} \rightarrow \mathbb{C}$ are defined by

$$
\mathcal{C}(f v):=(\mathcal{T} f) \times b \cdot B_{0} \sum_{k \in \mathscr{I}} R^{k} P^{k} v
$$

and

$$
\begin{aligned}
\widetilde{\mathcal{H}}^{\varepsilon}(f v):= & \cdot B_{0} \sum_{k \in \mathscr{I}} \sum_{\ell \in \mathscr{O}} e^{\frac{i}{\varepsilon}\left(\xi_{k}-\xi_{\ell}\right)}(\mathcal{T} f)\left(t-\alpha_{k, \ell}, x_{1}-\beta_{k, \ell}\right) R^{\ell} P^{\ell} \phi_{1} B_{1} P^{k} v \\
& +b \cdot B_{0} \sum_{k \in \mathscr{I}} \sum_{\ell \in \mathscr{O}} e^{\frac{i}{\varepsilon}\left(\xi_{k}-\xi_{\ell}\right)}(\mathcal{T} f)\left(t-\alpha_{k, \ell}, x_{1}-\beta_{k, \ell}\right) P^{\ell} \phi_{1} B_{1} R^{k} P^{k} v \\
& -b \cdot B_{0} \sum_{k \in \mathscr{I}} \sum_{\ell \in \mathscr{O}} \frac{e^{\frac{i}{\varepsilon}\left(\xi_{k}-\xi_{\ell}\right)}}{\mathbf{v}_{k, 2}}\left(\mathcal{T}^{2} f\right)\left(t-\alpha_{k, \ell}, x_{1}-\beta_{k, \ell}\right) P^{\ell} \phi_{1} B_{1} \widetilde{Q}^{k} Q^{k} R^{k} P^{k} v \\
& +b \cdot B_{0} \sum_{k \in \mathscr{I}} \sum_{\ell \in \mathscr{O}} \frac{e^{\frac{i}{\varepsilon}\left(\xi_{k}-\xi_{\ell}\right)}}{\mathbf{v}_{\ell, 2}}\left(\mathcal{T}^{2} f\right)\left(t-\alpha_{k, \ell}, x_{1}-\beta_{k, \ell}\right) \widetilde{Q}^{\ell} Q^{\ell} R^{\ell} P^{\ell} \phi_{1} B_{1} P^{k} v \\
& -b \cdot B_{0} \sum_{k \in \mathscr{I}} \sum_{\ell \in \mathscr{O}} \sum_{\ell^{\prime} \in \mathscr{O}} e^{\frac{i}{\varepsilon}\left(\xi_{k}-\xi_{\ell}\right)}(\mathcal{T} f)\left(t-\alpha_{k, \ell}, x_{1}-\beta_{k, \ell}\right) P^{\ell} \phi_{1} B_{1} P^{\ell^{\prime}} \phi_{1} B_{1} R^{k} P^{k} v .
\end{aligned}
$$

The definitions of $\mathcal{C}$ and $\widetilde{\mathcal{H}}^{\varepsilon}$ in themselves are of little interest. The only point to keep in mind is that $\mathcal{C}$ evaluates at time $t$ whereas $\widetilde{\mathcal{H}}^{\varepsilon}$ evaluates at times $t-\alpha_{k, \ell}$ in particular for any $m \in \mathbb{N}$ we have that $\widetilde{\mathcal{H}}^{\varepsilon}\left(\mathscr{P}_{m}\right) \subset \mathscr{P}_{m-1}$.

In 80 all the terms that depend directly on $\varrho_{K}^{\varepsilon}$ have been treated. Indeed the right hand side only depends on the $\varrho_{m}^{\varepsilon}, m \in \llbracket 0, K+1 \rrbracket \backslash\{K\}$ and $\nu_{K+1}^{\varepsilon}$, while the left hand side depends on the $\nu_{m}^{\varepsilon}$ for $m \in \llbracket 1, K \rrbracket$.

However from the first reformulation we can make the terms depending on the $\nu_{m}^{\varepsilon}$ for $m \in \llbracket 1, K \rrbracket$ more explicit in terms of $\varrho_{K}^{\varepsilon}$ it is the aim of the following. 
- "Hidden" terms coming from $\nu_{K}^{\varepsilon}$ Reiterating ${ }^{10}$ the same computations as the ones performed to deal with the terms depending directly on $\varrho_{K}^{\varepsilon}$ in $[76$ we can write 80 under the form:

$$
\begin{gathered}
i\left(\mathcal{C}-\widetilde{\mathcal{H}}^{\varepsilon}\right)\left(\varrho_{K}^{\varepsilon} e\right)+i\left(\mathcal{C}-\widetilde{\mathcal{H}}^{\varepsilon}\right)\left(\nu_{K}^{\varepsilon} v_{K, *}\right)= \\
-b \cdot B_{0} \sum_{m=1}^{K-1} \mathbb{F}_{K+1, m, *}^{\varepsilon}-b \cdot B_{0} \sum_{m=1}^{K-1} \mathbb{G}_{K+1, m, *}^{\varepsilon} \\
+\widetilde{\mathcal{R}}^{\varepsilon}\left(\varrho_{K+1}^{\varepsilon} e\right)+\widetilde{\mathcal{R}}^{\varepsilon}\left(\nu_{K+1}^{\varepsilon} v_{K+1, *}^{K-1}\right) .
\end{gathered}
$$

Because of Proposition 4.4 (applied with $n=K$ ) combined to the linearity of $\mathcal{C}$ and $\tilde{\mathcal{H}}$, we can write:

$$
\begin{aligned}
i\left(\mathcal{C}-\widetilde{\mathcal{H}}^{\varepsilon}\right)\left(\nu_{K}^{\varepsilon} v_{K, *}\right)= & i \sum_{m=0}^{K-1} \sum_{j=0}^{m} \mathcal{C}\left(\mathcal{S}_{*}^{\varepsilon}\right)^{j} \mathscr{W}_{K, m}^{\varepsilon}-i \sum_{m=1}^{K-1} \sum_{j=0}^{m-1} \widetilde{\mathcal{H}}^{\varepsilon}\left(\mathcal{S}_{*}^{\varepsilon}\right)^{j} \mathscr{W}_{K, m}^{\varepsilon} \\
& +i \sum_{j=1}^{K} \mathcal{C}\left(\mathcal{S}_{*}^{\varepsilon}\right)^{j} \varrho_{K}^{\varepsilon} e-i \sum_{j=1}^{K-1} \widetilde{\mathcal{H}}^{\varepsilon}\left(\mathcal{S}_{*}^{\varepsilon}\right)^{j} \varrho_{K}^{\varepsilon} e
\end{aligned}
$$

where we recall that the $\mathscr{W}_{K, m}^{\varepsilon}$ are linear operators depending on $\varrho_{m}^{\varepsilon}$ and such that $\mathscr{W}_{K, m}^{\varepsilon} \in \mathscr{P}_{m}$. Consequently we write 84 under the form:

$$
\begin{aligned}
i\left(\mathcal{C}-\overline{\mathcal{H}}^{\varepsilon}\right)\left(\varrho_{K}^{\varepsilon} e\right)= & b \cdot g-b \cdot B_{0} \sum_{m=0}^{K-1} \mathbb{F}_{K+1, m}^{\varepsilon}-b \cdot B_{0} \sum_{m=0}^{K-1} \mathbb{G}_{K+1, m}^{\varepsilon} \\
& -i b \cdot B_{0} \sum_{m=0}^{K-1} \sum_{j=0}^{m} \mathcal{C}\left(\mathcal{S}_{*}^{\varepsilon}\right)^{j} \mathscr{W}_{K, m}^{\varepsilon}+i b \cdot B_{0} \sum_{m=1}^{K-1} \sum_{j=0}^{m-1} \widetilde{\mathcal{H}}^{\varepsilon}\left(\mathcal{S}_{*}^{\varepsilon}\right)^{j} \mathscr{W}_{K, m}^{\varepsilon} \\
& -b \cdot B_{0} \sum_{m=1}^{K-1} \mathbb{F}_{K+1, m, *}^{\varepsilon}-b \cdot B_{0} \sum_{m=1}^{K-1} \mathbb{G}_{K+1, m, *}^{\varepsilon} \\
& +\widetilde{\mathcal{R}}^{\varepsilon}\left(\varrho_{K+1}^{\varepsilon} e\right)+\widetilde{\mathcal{R}}^{\varepsilon}\left(\nu_{K+1}^{\varepsilon} v_{K+1, *}\right),
\end{aligned}
$$

where we defined:

$$
\overline{\mathcal{H}}^{\varepsilon}=\widetilde{\mathcal{H}}^{\varepsilon}-i \sum_{j=1}^{K} \mathcal{C}\left(\mathcal{S}_{*}^{\varepsilon}\right)^{j}+i \sum_{j=1}^{K-1} \widetilde{\mathcal{H}}^{\varepsilon}\left(\mathcal{S}_{*}^{\varepsilon}\right)^{j},
$$

and in particular we remark, because the sums in the right hand side in this definition start at $j=1$ that $\overline{\mathcal{H}}^{\varepsilon}\left(\mathscr{P}_{m}\right) \subset \mathscr{P}_{m-1}$.

The left hand side of $(85)$ only depends on $\varrho_{K}^{\varepsilon}$. The two first lines in the right hand side only depend on the $\varrho_{m}^{\varepsilon}$ for $m \in \llbracket 0, K-1 \rrbracket$ and the third one on the $\nu_{m}^{\varepsilon}$ for $m \in \llbracket 1, K-1 \rrbracket$. Consequently in order to obtain an equation depending on the $\varrho_{m}^{\varepsilon}$ only we express the third line of the right hand side of $\varrho_{m}^{\varepsilon}$ only.

From Proposition 4.4 it turns out that (recall the slight abuse of notations $\mathbb{F}_{K+1, m, *}^{\varepsilon}=\mathbb{F}_{K+1, m, *}^{\varepsilon}\left[\nu_{m}^{\varepsilon} v_{m, *}\right]$

\footnotetext{
${ }^{10}$ Note that the terms $\varrho_{K}^{\varepsilon}$ and $\nu_{K}^{\varepsilon}$ play exactly the same role as in 61, 62, 65, and 66.
} 
and $\left.\mathbb{G}_{K+1, m, *}^{\varepsilon}=\mathbb{G}_{K+1, m, *}^{\varepsilon}\left[\nu_{m}^{\varepsilon} v_{m, *}\right]\right)$

$$
\begin{aligned}
-b \cdot B_{0} \sum_{m=1}^{K-1} \mathbb{F}_{K+1, m, *}^{\varepsilon}= & -b \cdot B_{0} \sum_{m=1}^{K-1} \sum_{p=0}^{m-1} \sum_{j=0}^{p} \mathbb{F}_{K+1, m, *}^{\varepsilon}\left[\left(\mathcal{S}_{*}^{\varepsilon}\right)^{j} \mathscr{W}_{m, p}^{\varepsilon}\right] \\
& -b \cdot B_{0} \sum_{m=1}^{K-1} \sum_{j=1}^{m} \mathbb{F}_{K+1, m, *}^{\varepsilon}\left[\left(\mathcal{S}_{*}^{\varepsilon}\right)^{j} \varrho_{m}^{\varepsilon} e\right], \\
-b \cdot B_{0} \sum_{m=1}^{K-1} \mathbb{G}_{K+1, m, *}^{\varepsilon}= & -b \cdot B_{0} \sum_{m=1}^{K-1} \sum_{p=0}^{m-1} \sum_{j=0}^{p} \mathbb{G}_{K+1, m, *}^{\varepsilon}\left[\left(\mathcal{S}_{*}^{\varepsilon}\right)^{j} \mathscr{W}_{m, p}^{\varepsilon}\right] \\
& -b \cdot B_{0} \sum_{m=1}^{K-1} \sum_{j=1}^{m} \mathbb{G}_{K+1, m, *}^{\varepsilon}\left[\left(\mathcal{S}_{*}^{\varepsilon}\right)^{j} \varrho_{m}^{\varepsilon} e\right], .
\end{aligned}
$$

Consequently 85 can be rewritten under the form:

$$
i\left(\mathcal{C}-\overline{\mathcal{H}}^{\varepsilon}\right)\left(\varrho_{K}^{\varepsilon} e\right)=b \cdot g+\sum_{m=0}^{K-1} \widetilde{\mathscr{X}_{K, m}^{\varepsilon}}+\widetilde{\mathcal{R}}^{\varepsilon}\left(\varrho_{K+1}^{\varepsilon} e\right)+\widetilde{\mathcal{R}}^{\varepsilon}\left(\nu_{K+1}^{\varepsilon} v_{K+1, *}\right),
$$

where for all $m \in \llbracket 0, K-1 \rrbracket, \widetilde{\mathscr{X}_{K, m}^{\varepsilon}}$ is a linear operator that only depends on $\varrho_{m}^{\varepsilon}$ satisfying that $\widetilde{\mathscr{X}_{K, m}^{\varepsilon}} \in \mathscr{P}_{m}$. These operators are explicitly given by:

$$
\begin{aligned}
\widetilde{\mathscr{X}_{K, m}^{\varepsilon}}:=b \cdot B_{0}( & \underbrace{-\mathbb{F}_{K+1, m}^{\varepsilon}-\mathbb{G}_{K+1, m}^{\varepsilon}}_{\in \mathscr{P}_{m}}-i \sum_{j=0}^{m} \underbrace{\mathcal{C}\left(\mathcal{S}_{*}^{\varepsilon}\right)^{j} \mathscr{W}_{K, m}^{\varepsilon}}_{\in \mathscr{P}_{m-j}}+i \sum_{j=0}^{m-1} \underbrace{\widetilde{\mathcal{H}}^{\varepsilon}\left(\mathcal{S}_{*}^{\varepsilon}\right)^{j} \mathscr{W}_{K, m}^{\varepsilon}}_{\in \mathscr{P}_{m-1-j}} \\
& -\sum_{p=0}^{m-1} \sum_{j=0}^{p} \underbrace{\left(\mathbb{F}_{K+1, m, *}^{\varepsilon}+\mathbb{G}_{K+1, m, *}^{\varepsilon}\right)\left[\left(\mathcal{S}_{*}^{\varepsilon}\right)^{j} \mathscr{W}_{m, p}^{\varepsilon}\right]}_{\in \mathscr{P}_{m-j}} \\
& -\sum_{j=1}^{m} \underbrace{\left(\mathbb{F}_{K+1, m, *}^{\varepsilon}+\mathbb{G}_{K+1, m, *}^{\varepsilon}\right)\left[\left(\mathcal{S}_{*}^{\varepsilon}\right)^{j} \mathscr{W}_{m, p}^{\varepsilon}\right]}_{\in \mathscr{P}_{m-j-1}}) .
\end{aligned}
$$

Consequently the only term that does not depend on the $\varrho_{m}^{\varepsilon}, m \in \llbracket 0, K+1 \rrbracket$ in $(86)$ is the one depending on $\nu_{K+1}^{\varepsilon}$. The last step is just to reformulate this term.

- End of the reformulation: the term depending on $\nu_{K+1}^{\varepsilon}$ Exactly as it as been done for the terms depending on the $\nu_{m}^{\varepsilon}$, we use Proposition 4.4 in order to express the term $\widetilde{\mathcal{R}}^{\varepsilon}\left(\nu_{K+1}^{\varepsilon} v_{K+1, *}\right)$ in the right hand side of $(86)$ in terms of the $\varrho_{m}^{\varepsilon}$. From the linearity and the definition of $\widetilde{\mathcal{R}}^{\varepsilon}$ we have:

$$
\begin{aligned}
\widetilde{\mathcal{R}}^{\varepsilon}\left(\nu_{K+1}^{\varepsilon} v_{K+1, *}\right)= & \sum_{j=0}^{K-1} \underbrace{\widetilde{\mathcal{R}}^{\varepsilon}\left(\mathcal{S}_{*}^{\varepsilon}\right)^{j} \phi_{0, *} g}_{\in \mathscr{P}_{K-1-j}}+\sum_{m=1}^{K-1} \sum_{j=0}^{m-1} \underbrace{\widetilde{\mathcal{R}}^{\varepsilon}\left(\mathcal{S}_{*}^{\varepsilon}\right)^{j} \mathscr{W}_{K+1, m}^{\varepsilon}}_{\in \mathscr{P}_{m-j-1}}+\sum_{j=0}^{K-1} \underbrace{\widetilde{\mathcal{R}}^{\varepsilon}\left(\mathcal{S}_{*}^{\varepsilon}\right)^{j} \mathscr{W}_{K+1, K}^{\varepsilon}}_{\in \mathscr{P}_{K-j-1}} \\
& +\sum_{j=1}^{K-1} \underbrace{\widetilde{\mathcal{R}}^{\varepsilon}\left(\mathcal{S}_{*}^{\varepsilon}\right)^{j} \varrho_{K+1}^{\varepsilon} e}_{\in \mathscr{P}_{K-j-1}},
\end{aligned}
$$

so that we rewrite (86) in its final form:

$$
\left(I-\mathcal{H}_{K}^{\varepsilon}\right)\left(\varrho_{K}^{\varepsilon} e\right)=-i \underbrace{\widetilde{\mathcal{C}}(b \cdot g)}_{\in \mathscr{P}_{K}}+\mathscr{E}_{g}+\sum_{m=0}^{K-1} \mathscr{X}_{K, m}^{\varepsilon}+\mathcal{R}^{\varepsilon}\left(\varrho_{K+1}^{\varepsilon} e\right)
$$


where for a scalar function $f$ we defined

$$
\widetilde{\mathcal{C}} f:=\left(b \cdot B_{0} \sum_{k \in \mathscr{I}} R^{k} e_{k}\right)^{-1} \mathcal{T}^{-1} f e
$$

and 11

$$
\begin{aligned}
\mathcal{H}_{K}^{\varepsilon} & :=\widetilde{\mathcal{C}}\left(\overline{\mathcal{H}}^{\varepsilon}-i \sum_{j=0}^{K-1} \widetilde{\mathcal{R}}^{\varepsilon}\left(\mathcal{S}_{*}^{\varepsilon}\right)^{j} \mathscr{W}_{K+1, K}^{\varepsilon}[\cdot]\right) \text { is s.t. } \forall m \in \mathbb{N}, \mathcal{H}^{\varepsilon}\left(\mathscr{P}_{m}\right) \subset \mathscr{P}_{m-1}, \\
\mathcal{R}^{\varepsilon} & :=-i \widetilde{\mathcal{C}}\left(\widetilde{\mathcal{R}}^{\varepsilon}+\sum_{j=1}^{K-1} \widetilde{\mathcal{R}}^{\varepsilon}\left(\mathcal{S}_{*}^{\varepsilon}\right)^{j}\right) \text { is s.t. } \forall m \in \mathbb{N}, \mathcal{R}^{\varepsilon}\left(\mathscr{P}_{m}\right) \subset \mathscr{P}_{m-1}, \\
\mathscr{E}_{g} & :=-i \sum_{j=0}^{K-1} \underbrace{\widetilde{\mathcal{C}}\left(\widetilde{\mathcal{R}}^{\varepsilon}\left(\mathcal{S}_{*}^{\varepsilon}\right)^{j} \phi_{0, *} g\right)}_{\in \mathscr{P}_{K-j-1}} \in \mathscr{P}_{K-1},
\end{aligned}
$$

and where for all $m \in \llbracket 0, K-1 \rrbracket, \mathscr{X}_{K, m}^{\varepsilon}$ only depends (linearly) on $\varrho_{m}^{\varepsilon}, \mathscr{X}_{K, m}^{\varepsilon} \in \mathscr{P}_{m}$ is given by:

$$
\mathscr{X}_{K, m}^{\varepsilon}=-i \widetilde{\mathcal{C}}\left(\widetilde{\mathscr{X}_{K, m}}+\sum_{j=0}^{K-1} \widetilde{\mathcal{R}}^{\varepsilon}\left(\mathcal{S}_{*}^{\varepsilon}\right)^{j} \mathscr{W}_{K+1, m}^{\varepsilon}\right) \text {. }
$$

Because the operator $\mathcal{H}_{K}^{\varepsilon}$, "costs" (in terms of time) at least the time needed to performed a full regeneration of self-interacting phases, we can inverse $\left(I-\mathcal{H}_{K}^{\varepsilon}\right)$ from its Neumann series expansion. Consequently, (88) becomes:

$$
\varrho_{K}^{\varepsilon} e=-i \sum_{j_{K}=0}^{K}\left(\mathcal{H}_{K}^{\varepsilon}\right)^{j_{K}} \widetilde{\mathcal{C}}(b \cdot g)+\sum_{j_{K}=0}^{K-1}\left(\mathcal{H}_{K}^{\varepsilon}\right)^{j_{K}} \mathscr{E}_{g}+\sum_{m=0}^{K-1} \sum_{j_{K}=0}^{m}\left(\mathcal{H}_{K}^{\varepsilon}\right)^{j_{K}} \mathscr{X}_{K, m}^{\varepsilon}+\sum_{j_{K}=0}^{K-1}\left(\mathcal{H}_{K}^{\varepsilon}\right)^{j_{K}} \mathcal{R}^{\varepsilon}\left(\varrho_{K+1}^{\varepsilon} e\right),
$$

which is an equation determining $\varrho_{K}^{\varepsilon}$ in terms of the $\varrho_{m}^{\varepsilon}$ for $m \in \llbracket 0, k-1 \rrbracket \cup\{K+1\}$.

The generic equation for $\varrho_{n}^{\varepsilon}$; last reformulation The aim of this paragraph is to show that the unknown component of the trace $\varrho_{K-1}^{\varepsilon}$ can be express in terms of the $\varrho_{m}^{\varepsilon}$ for $m \in \llbracket 0, K-2 \rrbracket \cup\{K+1\}$

Proposition 4.5 There exist a linear operator $\mathcal{H}_{K-1}^{\varepsilon}$ satisfying that for all $m \in \llbracket 0, K \rrbracket, \mathcal{H}_{K-1}^{\varepsilon}\left(\mathscr{P}_{m}\right) \subset$ $\mathscr{P}_{m-1}$ and for all $m \in \llbracket 0, K-2 \rrbracket$ a linear operator $\mathscr{X}_{K-1, m}^{\varepsilon}$ (depending only on $\varrho_{m}^{\varepsilon}$ ) satisfying that $\mathscr{X}_{K-1, m}^{\varepsilon} \in$ $\mathscr{P}_{m}$ such that we have the equality:

$$
\begin{aligned}
\varrho_{K-1}^{\varepsilon} e= & -i \sum_{j_{K}+j_{K-1}=0}^{K-1}\left(\mathcal{H}_{K-1}^{\varepsilon}\right)^{j_{K-1}} \mathcal{R}^{\varepsilon}\left(\mathcal{H}_{K}^{\varepsilon}\right)^{j_{K}} \widetilde{\mathcal{C}}(b \cdot g)+\sum_{j_{K}+j_{K-1}=0}^{K-2}\left(\mathcal{H}_{K-1}^{\varepsilon}\right)^{j_{K-1}} \mathcal{R}^{\varepsilon}\left(\mathcal{H}_{K}^{\varepsilon}\right)^{j_{K}} \mathscr{E}_{g} \\
& +\sum_{m=0}^{K-2} \sum_{j_{K-1}=0}^{m}\left(\mathcal{H}_{K-1}^{\varepsilon}\right)^{j_{K-1}} \underline{\mathscr{X}}_{K-1, m}^{\varepsilon}+\sum_{j_{K}+j_{K-1}=0}^{K-2}\left(\mathcal{H}_{K-1}^{\varepsilon}\right)^{j_{K-1}} \mathcal{R}^{\varepsilon}\left(\mathcal{H}_{K}^{\varepsilon}\right)^{j_{K}} \mathcal{R}^{\varepsilon}\left(\varrho_{K+1}^{\varepsilon} e\right) .
\end{aligned}
$$

Proof : If we reiterate exactly the same computations as the ones performed to obtain the equation (86) but this time with an initialization at $n=K-1$ in 75 we can show that there exist an operator $\mathcal{H}_{K-1}^{\mathcal{E}, \sharp}$ satisfying that for all $m \in \llbracket 0, K \rrbracket, \mathcal{H}_{K-1}^{\varepsilon, \sharp}\left(\mathscr{P}_{m}\right) \subset \mathscr{P}_{m-1}$ and for all $m \in \llbracket 0, K-2 \rrbracket$ linear operators $\mathscr{X}_{K-1, m}^{\varepsilon, \sharp}$ (only depending on the $\varrho_{m}^{\varepsilon}$ ) satisfying $\mathscr{X}_{K-1, m}^{\varepsilon, \sharp} \in \mathscr{P}_{m}$ such that

$$
\left(I-\mathcal{H}_{K-1}^{\varepsilon, \sharp}\right)\left(\varrho_{K-1}^{\varepsilon} e\right)=\sum_{m=0}^{K-2} \underline{\mathscr{X}}_{K-1, m}^{\varepsilon, \sharp}+\mathcal{R}^{\varepsilon}\left(\varrho_{K}^{\varepsilon} e\right) .
$$

\footnotetext{
${ }^{11}$ Note that because of the terms depending on $\mathscr{W}_{K+1, K}^{\varepsilon}$ in its definition, $\mathcal{H}_{K}^{\varepsilon}$ effectively depends on the choice $n=K+1$ in the previous computations. The subscript $K$ in the notation is thus there to highlight this dependency, because the operator to be inverted to determine $\varrho_{m}^{\varepsilon}$ will change with $m$. It was not the case for $\mathscr{S}_{*}^{\varepsilon}$
} 
Indeed, compared to the computations leading to $(86)$ the initialization at $n=K-1$ in $(75)$ makes the two terms depending on the source term in the right hand side of (86) disappear (because, for $n=K$, they come from the Kronecker symbol $\delta_{n, K}$ in the right hand of 75 and from the term depending on $\left.\nu_{K+1}^{\varepsilon}\right)$.

However if we define

$$
\mathcal{H}_{K-1}^{\varepsilon, \sharp}:=\widetilde{\mathcal{C}}\left(\overline{\mathcal{H}}^{\varepsilon}-i \sum_{j=0}^{K-2} \widetilde{\mathcal{R}}^{\varepsilon}\left(\mathcal{S}_{*}^{\varepsilon}\right)^{j} \mathscr{W}_{K, K-1}^{\varepsilon}\right) \text { and } \mathscr{X}_{K-1, m}^{\varepsilon, \sharp}:=-i \widetilde{\mathcal{C}}\left(\widetilde{\mathscr{X}_{K-1, m}}+\sum_{j=0}^{K-2} \widetilde{\mathcal{R}}^{\varepsilon}\left(\mathcal{S}_{*}^{\varepsilon}\right)^{j} \mathscr{W}_{K, m}^{\varepsilon}\right)
$$

that is that we essentially take the same definitions (up to the first subscript) as for $\mathcal{H}_{K}^{\varepsilon}$ and $\mathscr{X}_{K, m}^{\varepsilon}$ (see $(90)$ and (92) then (95) holds.

Indeed from its definition (see (91)) it is clear that $\mathcal{R}^{\varepsilon}$ does not depend on the initialization.

From (93) combined with the fact for all $m \in \llbracket 0, K \rrbracket, \mathcal{R}^{\varepsilon}\left(\mathscr{P}_{m}\right) \subset \mathscr{P}_{m-1}$ it turns out that:

$$
\begin{aligned}
\mathcal{R}^{\varepsilon}\left(\varrho_{K}^{\varepsilon} e\right)= & -i \sum_{j_{K}=0}^{K-1} \mathcal{R}^{\varepsilon}\left(\mathcal{H}_{K}^{\varepsilon}\right)^{j_{K}} \widetilde{\mathcal{C}}(b \cdot g)+\sum_{j_{K}=0}^{K-2} \mathcal{R}^{\varepsilon}\left(\mathcal{H}_{K}^{\varepsilon}\right)^{j_{K}} \mathscr{E}_{g}+\sum_{m=1}^{K-1} \sum_{j_{K}=0}^{m-1} \mathcal{R}^{\varepsilon}\left(\mathcal{H}_{K}^{\varepsilon}\right)^{j_{K}} \underline{\mathscr{X}}_{K, m}^{\varepsilon} \\
& +\sum_{j_{K}=0}^{K-2} \mathcal{R}^{\varepsilon}\left(\mathcal{H}_{K}^{\varepsilon}\right)^{j_{K}} \mathcal{R}^{\varepsilon}\left(\varrho_{K+1}^{\varepsilon} e\right)
\end{aligned}
$$

equation that we can use in the last term of the right hand side of 95 to rewrite this equation under the form:

$$
\begin{aligned}
\left(I-\mathcal{H}_{K-1}^{\varepsilon}\right)\left(\varrho_{K-1}^{\varepsilon} e\right)= & -i \sum_{j_{K}=0}^{K-1} \mathcal{R}^{\varepsilon}\left(\mathcal{H}_{K}^{\varepsilon}\right)^{j_{K}} \widetilde{\mathcal{C}}(b \cdot g)+\sum_{j_{K}=0}^{K-2} \mathcal{R}^{\varepsilon}\left(\mathcal{H}_{K}^{\varepsilon}\right)^{j_{K}} \mathscr{E}_{g}+\sum_{m=0}^{K-2} \underline{\mathcal{X}}_{K-1, m}^{\varepsilon} \\
& +\sum_{j_{K}=0}^{K-2} \mathcal{R}^{\varepsilon}\left(\mathcal{H}_{K}^{\varepsilon}\right)^{j_{K}} \mathcal{R}^{\varepsilon}\left(\varrho_{K+1}^{\varepsilon} e\right),
\end{aligned}
$$

where we introduced

$$
\begin{array}{r}
\mathcal{H}_{K-1}^{\varepsilon}:=\mathcal{H}_{K-1}^{\varepsilon, \sharp}+\sum_{j=0}^{K-2} \mathcal{R}^{\varepsilon}\left(\mathcal{H}_{K}^{\varepsilon}\right)^{j} \mathscr{X}_{K, K-1}^{\varepsilon}, \\
\forall m \in \llbracket 0, K-2 \rrbracket, \mathscr{X}_{K-1, m}^{\varepsilon}:=\mathscr{X}_{K-1, m}^{\varepsilon, \sharp}+\sum_{j=0}^{m-1} \mathcal{R}^{\varepsilon}\left(\mathcal{H}_{K}^{\varepsilon}\right)^{j} \mathscr{X}_{K, m}^{\varepsilon}
\end{array}
$$

From their definitions it is clear that $\mathcal{H}_{K-1}^{\varepsilon}$ and $\mathscr{X}_{K-1, m}^{\varepsilon}$ satisfy the required properties. Because $\mathcal{H}_{K-1}^{\varepsilon}\left(\mathscr{P}_{m}\right) \subset \mathscr{P}_{m-1}$, we can invert $\left(I-\mathcal{H}_{K-1}^{\varepsilon}\right)$ by taking its Neumann series expansion and (94) follows.

By iteration (or more precisely, several applications of Proposition 4.5) we then obtain

Proposition 4.6 For all $q \in \llbracket 0, K \rrbracket$ there exist linear operators $\mathcal{H}_{K-q}^{\varepsilon}$ satisfying that for all $m \in \llbracket 0, K \rrbracket$, $\mathcal{H}_{K-q}^{\varepsilon}\left(\mathscr{P}_{m}\right) \subset \mathscr{P}_{m-1}$ and for all $m \in \llbracket 0, K-q-1 \rrbracket$ a linear operator $\mathscr{X}_{K-q, m}^{\varepsilon}$ (depending only on $\varrho_{m}^{\varepsilon}$ ) satisfying that $\mathscr{X}_{K-1, m}^{\varepsilon} \in \mathscr{P}_{m}$ such that if we introduce $j=\left(j_{K}, j_{K-1}, \ldots, j_{K-q}\right) \in \mathbb{N}^{q+1}$ and defin $£^{12}$

$$
\mathbb{H}_{j}^{\varepsilon}:=\left(\bigcirc_{r=-q}^{-1}\left(\mathcal{H}_{K+r}^{\varepsilon}\right)^{j_{K+r}} \mathcal{R}^{\varepsilon}\right)\left(\mathcal{H}_{K}^{\varepsilon}\right)^{j_{K}}
$$

\footnotetext{
${ }^{12}$ We use the convention $\bigodot_{r=0}^{q} A_{r}=A_{0} \circ A_{1} \circ \cdots \circ A_{q}$ if $q \geq 0$ and $\bigodot_{r=0}^{q} A_{r}=I$ if $q<0$.
} 
then we have the equality:

$$
\begin{aligned}
\varrho_{K-q}^{\varepsilon} e= & -i \sum_{|j| \leq K-q} \mathbb{H}_{j}^{\varepsilon} \widetilde{\mathcal{C}}(b \cdot g)+\sum_{|j| \leq K-q-1} \mathbb{H}_{j}^{\varepsilon} \mathscr{E}_{g}+\sum_{m=0}^{K-q-1} \sum_{j_{K-q}=0}^{m}\left(\mathcal{H}_{K-q}^{\varepsilon}\right)^{j_{K-q}} \underline{\mathscr{X}}_{K-q, m}^{\varepsilon} \\
& +\sum_{|j| \leq K-q-1} \mathbb{H}_{j}^{\varepsilon} \mathcal{R}^{\varepsilon}\left(\varrho_{K+1}^{\varepsilon} e\right),
\end{aligned}
$$

where $|j|:=\sum_{r=0}^{q} j_{K-r}$.

\subsubsection{The explicit expression of $\varrho_{0}^{\varepsilon}$, end of the determination of the leading order amplitudes}

From Proposition 4.6 (applied to $q=K$ ) we directly obtain the explicit expression of $\varrho_{0}^{\varepsilon}$ in terms of the boundary source term. More precisely, we have

Proposition 4.7 The unknown trace component $\varrho_{0}^{\varepsilon}$ e is given by

$$
\varrho_{0}^{\varepsilon} e=-i\left(\mathcal{R}^{\varepsilon}\right)^{K} \widetilde{\mathcal{C}}(b \cdot g)=(-i)^{K+1}\left(\widetilde{\mathcal{C}} \widetilde{\mathcal{R}}^{\varepsilon}\right)^{K} \widetilde{\mathcal{C}}(b \cdot g),
$$

where we recall that $\widetilde{\mathcal{R}}^{\varepsilon}$ is defined in $(77)$ and $\widetilde{C}$ is defined in $(89)$.

Moreover there exist source terms $g \in \mathscr{P}_{K}$ such that $\varrho_{0}^{\varepsilon}$ is not identically zero.

Proof : We apply Proposition 4.6 with $q=K$. The only non trivial sum in the right hand side of (98) is the first one and it is restricted to $j=0_{\mathbb{N}^{K+1}}$. Consequently $\varrho_{0}^{\varepsilon} e=-i \mathbb{H}_{0^{\mathbb{N}} K+1} \widetilde{\mathcal{C}}(b \cdot g)$ and the first equality in (99) follows from the definition of $\mathbb{H}_{j}$ (see (97)).

To justify the second inequality recall that by definition:

$$
\mathcal{R}^{\varepsilon}=-i \widetilde{\mathcal{C}}\left(\widetilde{\mathcal{R}}^{\varepsilon}+\sum_{j=1}^{K-1} \widetilde{\mathcal{R}}^{\varepsilon}\left(\mathcal{S}_{*}^{\varepsilon}\right)^{j}\right):=-i \widetilde{\mathcal{C}}\left(\widetilde{\mathcal{R}}^{\varepsilon}+\mathcal{V}^{\varepsilon}\right) .
$$

So for all $m \in \llbracket 0, K \rrbracket, \mathcal{R}^{\varepsilon}\left(\mathscr{P}_{m}\right) \subset \mathscr{P}_{m-1}$ and $\mathcal{V}^{\varepsilon}\left(\mathscr{P}_{m}\right) \subset \mathscr{P}_{m-2}$ (because the sum defining $\mathcal{V}^{\varepsilon}$ starts at $j=1$ ). Consequently if we expand $\left(\mathcal{R}^{\varepsilon}\right)^{K}$ then all the terms containing at least one occurrence of $\mathcal{V}^{\varepsilon}$ vanish when they are applied to $\widetilde{\mathcal{C}}(b \cdot g)$. So the second equality in 99 is proven.

To conclude we have to justify that for some source terms $g$ we have that $\varrho_{0}^{\varepsilon}$ is not identically zero. Let for example $g:=\psi\left(t, x_{1}\right) b$, where $\psi \in \mathscr{D}(]-\infty, T[\times \mathbb{R})$ vanishes for negative times. Then we have $\mathscr{C}(b \cdot g)=$ $|b|^{2}\left(b \cdot B_{0} \sum_{k \in \mathscr{I}} R^{k} e_{k}\right)^{-1} \mathscr{T}^{-1} \psi \neq 0$. Because of the definition of $\widetilde{\mathcal{R}}^{\varepsilon}$ we can separate the functional part of $\mathscr{C}(b \cdot g)$ and the vectorial one and we just need to justify that $\widetilde{\mathcal{R}}^{\varepsilon}\left(\mathscr{T}^{-1} e\right) \neq 0$ which is generically true (and is always satisfied under Assumption 3.3 up to impose moreover that $\operatorname{ker} B_{0} \cap \operatorname{ker} B_{1}=\{0\}$ )

Proposition 4.7 then completes the construction of the leading order amplitudes. Indeed, the amplitudes $u_{0, k}^{\varepsilon}$ and $u_{0, \ell}^{\varepsilon}$ for $k \in \mathscr{I}, \ell \in \mathscr{O}$ can be explicitly expressed in terms of the source term $g$ by using (99) and $\nu_{0}^{\varepsilon} \equiv 0$ in $(30)$ and $(32)$.

\subsection{Determination of higher order amplitudes and summary}

In this paragraph we describe the main steps in the determination of the higher order amplitudes and we illustrate our discussion for the amplitudes of order one, namely the $u_{1, k}^{\varepsilon}$ for $k \in \mathscr{H}$.

First recall that as soon as $n \geq 1$, the amplitudes $u_{n, k}^{\varepsilon}$ are not polarized any more so that we have to determine $\left(I-P^{k}\right) u_{n, k}^{\varepsilon}$ and $P^{k} u_{n, k}^{\varepsilon}$ separately. 
- About the unpolarized part: because the leading order amplitudes are determined, the construction of the unpolarized part of the $u_{1, k}^{\varepsilon}$ for $k \in \mathscr{H}$ is automatic. Indeed, from 42 and 40 the $\left(I-P^{k}\right) u_{1, k}^{\varepsilon}$ are explicitly given in terms of $\varrho_{0}^{\varepsilon}$ which is known from Proposition 4.7 .

More generically, if all the $\varrho_{m}^{\varepsilon}$ and $\nu_{m}^{\varepsilon}$ are known for $m \in \llbracket 0, n-1 \rrbracket$, from Proposition 4.1 the same holds at the order $n$ (because $\mathscr{F}_{n, k, n p}^{\varepsilon}$ and $\mathscr{G}_{n, \ell, n p}^{\varepsilon}$ depend linearly on the $\varrho_{m}^{\varepsilon}$ and $\nu_{m}^{\varepsilon}$ for $m \in \llbracket 0, n-1 \rrbracket$ ).

- About the polarized part: From (47) and (49), the $P^{k} u_{1, k}^{\varepsilon}, k \in \mathscr{I}$ and the $P^{\ell} u_{1, \ell}^{\varepsilon}, \ell \in \mathscr{O}$ depend explicitly on $\varrho_{1}^{\varepsilon}, \nu_{1}^{\varepsilon}$ and $\varrho_{0}^{\varepsilon}$.

So that from Proposition 4.1 combined with (54) (which determines $\nu_{1}^{\varepsilon}$ in terms of $\varrho_{0}^{\varepsilon}$ and $\varrho_{1}^{\varepsilon}$ ) it is sufficient to construct $\varrho_{1}^{\varepsilon}$ to end the construction of the $P^{k} u_{1, k}^{\varepsilon}, k \in \mathscr{H}$.

To determine $\varrho_{1}^{\varepsilon}$ one may use Proposition 4.6 (with $q=K-1$ ) so that 98 determines $\varrho_{1}^{\varepsilon}$ in terms of the source term $g$ (the two first term in the right hand side of $(98)$ ) and $\varrho_{0}^{\varepsilon}$ (the third term).

Unfortunately the last term in the right hand side of $(98)$ then becomes $\mathcal{R}^{K}\left(\varrho_{K+1}^{\varepsilon} e\right) \in \mathscr{P}_{0}$ (indeed, the amplitude of order one may perfectly a priori depend on the one of order $K+1$ which has made $K$ completes regenerations).

To overcome this difficulty, the simplest way is to start from $(75)$ with the initialization $n=K+1$ (recall that we used $n=K$ to obtain the relation on (93) ) and to reiterate the same computations/reformulations made to derive (93). This method leads to an equation of the form:

$$
\varrho_{K+1}^{\varepsilon} e=\mathscr{E}_{g, K+1}+\sum_{m=0}^{K} \sum_{j=0}^{m}\left(\mathcal{H}_{K+1}^{\varepsilon}\right)^{j}{\underline{X^{\varepsilon}}}_{K+1, m}+\sum_{j=0}^{K-1}\left(\mathcal{H}_{K+1}^{\varepsilon}\right){ }^{j} \mathcal{R}^{\varepsilon}\left(\varrho_{K+2}^{\varepsilon} e\right),
$$

where $\mathscr{E}_{g, K+1}$ is a term depending explicitly on $g$ and where the $\mathscr{X}_{K+1, m}^{\varepsilon}$ and $\mathcal{H}_{K+1}^{\varepsilon}$ have the same expressions as the $\mathscr{X}_{K, m}^{\varepsilon}$ and $\mathcal{H}_{K}^{\varepsilon}$ (see (87) and (90)) with the subscript $K$ replaced by $K+1$. Let us stress that the other occurrences of $K$ in (87) and (90) are consequences of the profile spaces so that they stay unchanged.

Similarly the last sum in the right hand side of 100 is still indexed by $j \in \llbracket 0, K-1 \rrbracket$ because $\varrho_{K+2}^{\varepsilon} \in \mathscr{P}_{K}$.

So reiterating the same computations as the ones performed to obtain Propositions 4.5 and 4.6 from 75 gives (up to some harmless modifications in the definitions of the $\mathbb{H}_{j}^{\varepsilon}$ and the $\mathscr{X}_{K, m}^{\varepsilon}$ ) the analogous of 998$)$ for $\varrho_{K+1}^{\varepsilon}$ that is

$$
\varrho_{K+1-q}^{\varepsilon} e=\widetilde{\mathscr{E}}_{g, K+1, q}+\sum_{m=0}^{K-q} \sum_{j_{K-q}=0}^{m}\left(\mathcal{H}_{K+1-q}^{\varepsilon}\right)^{j_{K-q}}{\underline{\mathscr{X}_{K+1-q, m}^{\varepsilon}}}^{\varepsilon} \sum_{|j| \leq K-q-1} \mathbb{H}_{j}^{\varepsilon} \mathcal{R}^{\varepsilon}\left(\varrho_{K+2}^{\varepsilon} e\right),
$$

where $\widetilde{\mathscr{E}}_{g, K+1, q}$ explicitly depends on the source term. So the choice $q=K$ in 101 gives the desired value of $\varrho_{1}^{\varepsilon}$ in terms of the source term $g$ and $\varrho_{0}^{\varepsilon}$ (coming from the only non zero term in the first sum of the right hand side of (101), namely $\mathscr{X}_{1,0}^{\varepsilon}$ ) only. Indeed the crucial point is that the contribution of $\varrho_{K+2}^{\varepsilon}$ in the right hand side of 101 is zero for $q=K$.

This completes the determination of $\varrho_{1}^{\varepsilon}$ and consequently the one of the polarized part of the amplitudes of order one.

More generically, Proposition 4.1 ensures that to conclude the construction of the $P^{k} u_{n, k}^{\varepsilon}, k \in \mathscr{H}$ it is sufficient to know the $\varrho_{m}^{\varepsilon}$ and the $\nu_{m}^{\varepsilon}$ for all $m \in \llbracket 0, n-1 \rrbracket$. Indeed the terms $\mathscr{F}_{n, k, p}^{\varepsilon}$ and $\mathscr{G}_{n, \ell, p}^{\varepsilon}$ depend on the $\varrho_{m}^{\varepsilon}$ and the $\nu_{m}^{\varepsilon}$ for all $m \in \llbracket 0, n-1 \rrbracket$ and the last terms in the right hand sides of (59), 60) only depend on $\varrho_{n}^{\varepsilon}$ and $\nu_{n}^{\varepsilon}$.

Thanks to Proposition 4.4 the construction of the $P^{k} u_{n, k}^{\varepsilon}, k \in \mathscr{H}$ is thus over if we know all the $\varrho_{m}^{\varepsilon}$ for $m \in \llbracket 0, n \rrbracket$. 


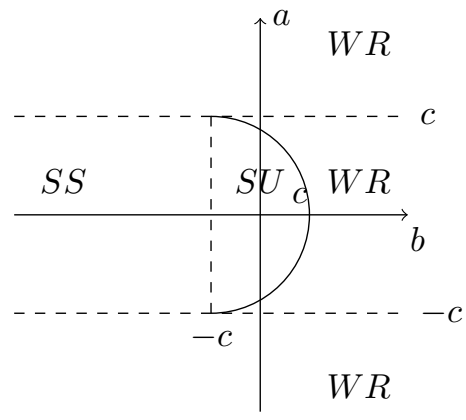

Figure 4: Influence of the parameters $(a, b)$ on the strong well-posedness of (102).

\section{Examples and comments}

\subsection{Examples}

Theorem 3.2 applies for example to the wave equation or the linearisation of Euler equations. Moreover these two examples show clearly that $W R$-boundary conditions for hyperbolic boundary value problems are far to be anecdotal.

The $2 d$-wave equation (see[BGRSZ02] and [CG10]) We consider the boundary value problem for the classical waves equation

$$
\begin{cases}\partial_{t t}^{2} u=c^{2} \Delta u & \text { in } \Omega_{T}, \\ \partial_{t} u_{\mid x_{2}=0}+a\left(\partial_{x_{2}} u\right)_{\mid x_{2}=0}+b\left(\partial_{x_{1}} u\right)_{\mid x_{2}=0}=g_{0} & \text { on } \partial \Omega_{0, T}, \\ u_{\mid t \leq 0}=0 & \text { on } \Gamma,\end{cases}
$$

and equipped with any boundary condition on the side $\partial \Omega_{1, T}$ such that the problem in the half-space $\left\{x_{2}<1\right\}$ is strongly well-posed. In 102 the parameter $a, b \in \mathbb{R}$ and the parameter $c>0$ stands for the speed of sound.

The scalar wave equation can be seen as a linear system of hyperbolic partial differential equations of order one and size $N=3$. The associated system is constantly hyperbolic and non characteristic.

The parameters such that the boundary condition on $\partial \Omega_{0, T}$ is in the $W R$-class are also known (we refer to [BGRSZ02] for the details of the computation) and are illustrate in Figure 4 (in which $S S$ (resp. $S U$ ) stands for the set of problems that are strongly well-posed (resp. ill-posed )).

Linearisation of Euler equation We consider the linearised Euler equation around a constant subsonic incoming (for the side $\partial \Omega_{0, T}$ ) state. This system reads

$$
\begin{cases}\partial_{t} u+A_{1} \partial_{1} u+A_{2} \partial_{2} u=f & \text { in } \Omega_{T}, \\ B_{0} u_{\mid x_{2}=0}=g_{0} & \text { on } \partial \Omega_{0, T}, \\ B_{1} u_{\mid x_{2}=1}=g_{1} & \text { on } \partial \Omega_{1, T}, \\ u_{\mid t \leq 0}=0 & \text { on } \Omega,\end{cases}
$$

where the matrices $A_{1}$ and $A_{2}$ are given by

$$
A_{1}=\left[\begin{array}{ccc}
0 & -1 & 0 \\
-1 & 0 & 0 \\
0 & 0 & 0
\end{array}\right], A_{2}=\left[\begin{array}{ccc}
M & 0 & -1 \\
0 & M & 0 \\
-1 & 0 & M
\end{array}\right]
$$

in which $M \in]-1,0[$ stands for the Mach number. The system 103 is constantly hyperbolic and noncharacteristic. The boundary matrix $B_{0}$ is taken under the form

$$
B_{0}:=\left[\begin{array}{lll}
1 & a & b
\end{array}\right] \text {, }
$$




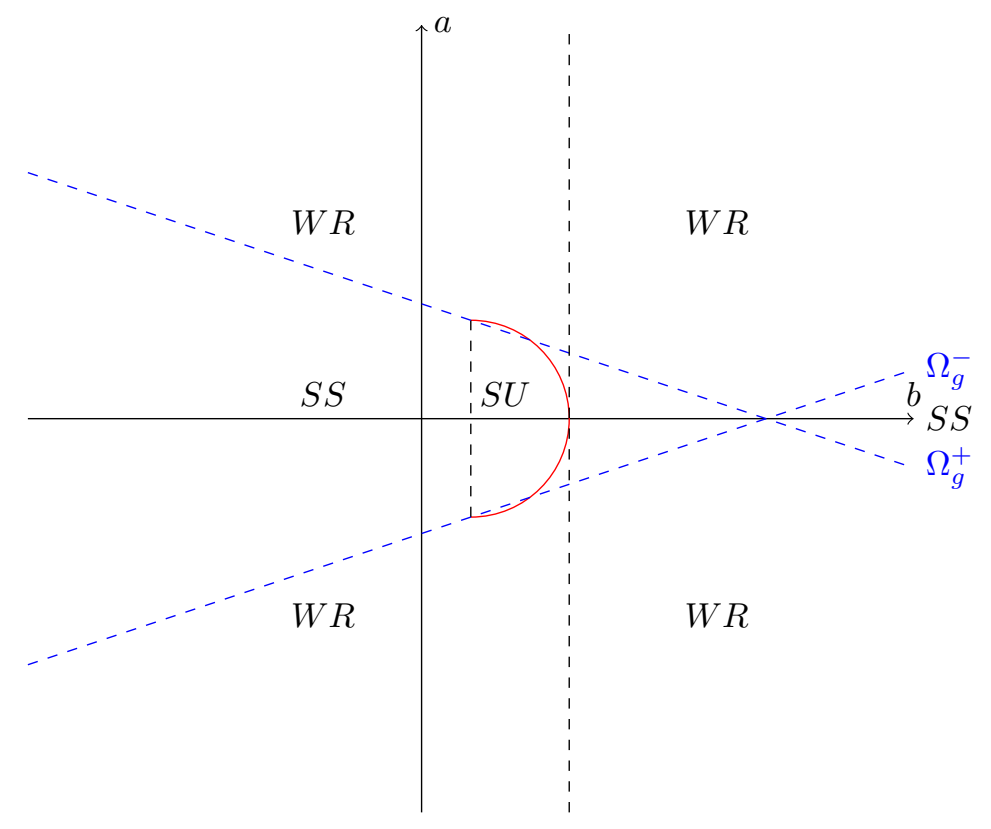

Figure 5: The influence of the boundary condition on the strong well-posedness of 103.

where $a, b \in \mathbb{R}$. The boundary matrix $B_{1} \in \mathbf{M}_{2 \times 3}(\mathbb{R})$ is assumed to be strictly dissipative (we refer for example to BGS07 for a precise definition) or at least to satisfy the uniform Kreiss-Lopatinskii condition.

Depending on the values of $a$ and $b$ we have the following behaviours for the class of belonging of (103) (see [Ben14] for the details of the computations). So that the boundary condition $B_{0}$ is in the $W R$ class and consequently Theorem 3.2 applies for all boundary parameters, $a, b$ such that $b \neq 1$, lying in the set

$$
\begin{aligned}
W R & :=\left\{(a, b) \in \mathbb{R}^{2} \backslash b<-M \text { and }|a|>\frac{1+M b}{\sqrt{1-M^{2}}}\right\} \cup\left\{(a, b) \in \mathbb{R}^{2} \backslash b<-\frac{1}{M} \text { and }|a|>-\frac{1+M b}{\sqrt{1-M^{2}}}\right\} . \\
& =\left\{(a, b) \in \mathbb{R}^{2} \backslash b<-M \text { and }|a|>\Omega_{g}^{+}\right\} \cup\left\{(a, b) \in \mathbb{R}^{2} \backslash b<\frac{1}{M} \text { and }|a|>\Omega_{g}^{-}\right\} .
\end{aligned}
$$

\subsection{Conclusion and comments}

In this article we have shown that if one of the boundary conditions of the strip degenerates in the $W R$-class then the loss of derivative of the solution with respect to the regularity of the source term on the boundary has to be at least bounded by below by a step function increasing with the final time of resolution.

The source of the instability is exactly the same as in the half-space but the geometry of the strip in itself permits to regenerate this instability an arbitrary number of time if the final time of resolution is large enough. In particular one can generically (unless that $B_{1}$ is particular enough to destroy the self-interaction phenomenon) not expect that a "good" choice of the boundary condition on $B_{1}$ compensate the instability generated by $B_{0}$.

On the one hand, one can not expect weak well-posedness in infinite time to hold. But on the other hand if the final time of resolution is fixed once for all then the construction of the geometric optics expansion exposed in Section 4 seems to indicate that the loss of regularity of the solution on the boundary in bounded by above by $\left[\frac{T}{\alpha}\right]+2$ so that it remains finite.

All the results above have been stated when the strip is defined by $\mathbb{R} \times] 0,1[$ in order to have a readable expression of the transport operator along the boundary $\partial \Omega_{0}$ namely $\mathcal{T}$. However compared to [Ben17. we 
do note used the precise value of the coefficient associated to $\partial_{1}$ to solve this transport equation. It seems to indicate that the results of this article can be mutadis mutandis extended to higher dimensional strips that is to say to $\left.\mathbb{R}^{d-1} \times\right] 0,1[$ for $d>2$.

In the half-space geometry it is also known that for a problem in the $W R$-class there is in addition of the loss of one derivative on the boundary one loss in the interior. Theorem 3.2 does not give any information about a lower bound on the loss of regularity in the interior.

However it seems that a construction of the geometric optics expansions for a non trivial source term in the interior can give essentially the same time depending loss of regularity in the interior.

At last, in this article we just consider the case of the degeneracy of the uniform Kreiss-Lopatinskii condition for a frequency of hyperbolic type. However other kind of degeneracies are possible depending on the type of the considered frequency. We give here some results and reasonable conjectures about the associated losses of derivatives for the other kind of frequency.

- $\zeta \in \Xi \backslash \Xi_{0}$. In this case the boundary value problem in the half-space to known to generates Hadamard instability and consequently the same should occur for the associated problem in the strip.

- $\underline{\zeta} \in \mathbb{E}$. In such a framework it has been shown by $\underline{\text { ST88 }}$ in the half-space is

$$
\|u\|_{L^{2}\left(\widetilde{\Omega}_{T}\right)}^{2}+\left\|u_{\mid x_{d}=0}\right\|_{H^{-1 / 2}\left(\partial \widetilde{\Omega}_{T}\right)}^{2} \leq C_{T}\left(\|f\|_{L^{2}\left(\widetilde{\Omega}_{T}\right)}^{2}+\|g\|_{H^{1 / 2}\left(\partial \widetilde{\Omega}_{T}\right)}^{2}\right),
$$

so that we have a loss of one derivative for the boundary term only.

In particular the problem is strongly well-posed if and only if it is homogeneous on the boundary. If it not the case any more then using the fact that elliptic modes are associated to exponentially decreasing with respect to the normal variable $x_{d}$, these wave packets can not propagate the singularity from one side of the boundary to the other and consequently the singularity is not amplified (see [Bena]). As a consequence the energy estimate in the strip geometry is the same as the one in the half-space that is to say a loss of a single derivative on the boundary $\partial \Omega_{0}$ (if it is this condition that does not satisfiy the uniform Kreiss-Lopatinskii condition):

$$
\begin{aligned}
\|u\|_{L^{2}\left(\Omega_{T}\right)}^{2}+\left\|u_{\mid x_{d}=0}\right\|_{H^{-1 / 2}\left(\partial \Omega_{0, T}\right)}^{2} & +\left\|u_{\mid x_{d}=1}\right\|_{L^{2}\left(\partial \Omega_{1, T}\right)}^{2} \\
& \leq C_{T}\left(\|f\|_{L^{2}\left(\Omega_{T}\right)}^{2}+\left\|g_{0}\right\|_{H^{1 / 2}\left(\partial \Omega_{0, T}\right)}^{2}+\left\|g_{1}\right\|_{L^{2}\left(\partial \Omega_{1, T}\right)}^{2}\right) .
\end{aligned}
$$

- $\underline{\zeta} \in \mathbb{M}$. In such a framework it has been shown in Cou02 that depending on the part of the stable subspace in which the uniform Kreiss-Lopantiskii breaks down the energy estimate in the half-space can be

$$
\|u\|_{L^{2}\left(\widetilde{\Omega}_{T}\right)}^{2}+\left\|u_{\mid x_{d}=0}\right\|_{H^{1 / 2}\left(\partial \widetilde{\Omega}_{T}\right)}^{2} \leq C_{T}\left(\|f\|_{\left.\left.L_{x_{d}}^{2}\left(H_{t, x^{\prime}}^{1 / 2}(]-\infty, T\right] \times \mathbb{R}^{d-1}\right)\right)}^{2}+\|g\|_{H^{1 / 2}\left(\partial \widetilde{\Omega}_{T}\right)}^{2}\right),
$$

if the uniform Kreiss-Lopatinskii condition breaks down for an elliptic mode and

$$
\|u\|_{L^{2}\left(\widetilde{\Omega}_{T}\right)}^{2}+\left\|u_{\mid x_{d}=0}\right\|_{H^{1 / 2}\left(\partial \widetilde{\Omega}_{T}\right)}^{2} \leq C_{T}\left(\|f\|_{\left.\left.L_{x_{d}}^{2}\left(H_{t, x^{\prime}}^{1}(]-\infty, T\right] \times \mathbb{R}^{d-1}\right)\right)}^{2}+\|g\|_{H^{1 / 2}\left(\partial \widetilde{\Omega}_{T}\right)}^{2}\right),
$$

if the uniform Kreiss-Lopatinskii condition is not satisfies on a hyperbolic mode. Consequently in the first case we observe a loss of one half of derivative in the interior and one derivative on the boundary while in the second case the losses are one in the interior and one on the boundary.

Once again using the fact that elliptic modes can not propagate the singularity the energy estimate for a degeneracy of the uniform Kreiss-Lopatinskii condition on an elliptic mode should be the same as the one in the half-space that is

$$
\begin{aligned}
\|u\|_{L^{2}\left(\Omega_{T}\right)}^{2}+ & \left\|u_{\mid x_{d}=0}\right\|_{L^{2}\left(\partial \Omega_{0, T}\right)}^{2}+\left\|u_{\mid x_{d}=1}\right\|_{L^{2}\left(\partial \Omega_{0, T}\right)}^{2} \\
& \leq C_{T}\left(\|f\|_{\left.\left.L_{x_{d}}^{2}\left(H_{t, x^{\prime}}^{1 / 2}(]-\infty, T\right] \times \mathbb{R}^{d-1}\right)\right)}^{2}+\left\|g_{0}\right\|_{H^{1 / 2}\left(\partial \Omega_{0, T}\right)}^{2}+\left\|g_{1}\right\|_{L^{2}\left(\partial \Omega_{1, T}\right)}^{2}\right),
\end{aligned}
$$

while if the uniform Kreiss-Lopatinskii condition degenerates for a hyperbolic mode, the singularity is transported and thus amplifies with time and we are in the framework studied in this article. 


\section{References}

[AM87] M. Artola and A. Majda. Nonlinear development of instabilities in supersonic vortex sheets. I. The basic kink modes. Phys. D, 28(3):253-281, 1987.

[Bena] A. Benoit. Weak well-posedness of hyperbolic boundary value problems in a strip: when instabilities do not meet the geometry (preprint) https://hal.archives-ouvertes.fr/hal-01783948v1,.

[Benb] A. Benoit. Wkb expansions for hyperbolic boundary value problems in a strip: selfinteraction meets strong well posedness (to appear in Journal of the Institute of Mathematics of Jussieu) https://hal.archives-ouvertes.fr/hal-01783948v1,.

[Ben14] A. Benoit. Geometric optics expansions for linear hyperbolic boundary value problems and optimality of energy estimates for surface waves. Differential Integral Equations, 27(5-6):531562,2014 .

[Ben17] A. Benoit. Geometric optics expansions for hyperbolic corner problems ii, huge amplification phenomenon. SIAM J., Math Anal, 49(5):3335-3395, 2017.

[BGRSZ02] S. Benzoni-Gavage, F. Rousset, D. Serre, and K. Zumbrun. Generic types and transitions in hyperbolic initial-boundary-value problems. Proc. Roy. Soc. Edinburgh Sect. A, 132(5):1073$1104,2002$.

[BGS07] S. Benzoni-Gavage and D. Serre. Multidimensional hyperbolic partial differential equations. Oxford Mathematical Monographs. Oxford University Press, 2007.

[CG10] J.-F. Coulombel and O. Guès. Geometric optics expansions with amplification for hyperbolic boundary value problems: linear problems. Ann. Inst. Fourier (Grenoble), 60(6):2183-2233, 2010 .

[Cou02] J.-F. Coulombel. Stabilité multidimensionnelle d'interfaces dynamiques. Application aux transitions de phase liquide-vapeur. PhD thesis, ENS Lyon, 2002.

[Cou05] J.-F. Coulombel. Well-posedness of hyperbolic initial boundary value problems. J. Math. Pures Appl. (9), 84(6):786-818, 2005.

[CSFM14] F. Colombini, D. Santo, F. Fanelli, and G. Metivier. The well-posedness issue in sobolev spaces for hyperbolic systems with zygmund-type coefficients. Communications in Partial Differential Equations, 40, 032014.

[CW17] J.-F. Coulombel and M. Williams. The mach stem equation and amplification in strongly nonlinear geometric optics. American Journal of Mathematics, 139(4):967-1046, 2017.

[Her63] R. Hersh. Mixed problems in several variables. J. Math. Mech., 12:317-334, 1963.

[Kre70] H.-O. Kreiss. Initial boundary value problems for hyperbolic systems. Comm. Pure Appl. Math., 23:277-298, 1970.

[Lax57] P. D. Lax. Asymptotic solutions of oscillatory initial value problems. Duke Math. J., 24:627-646, 1957.

[Les07] V. Lescarret. Wave transmission in dispersive media. Math. Models Methods Appl. Sci., 17(4):485-535, 2007.

[Maj88] A. Majda, M. Artola. Nonlinear geometric optics for hyperbolic mixed problems. In Analyse mathématique et applications, pages 319-356. Gauthier-Villars, 1988.

[Mar10] A. Marcou. Rigorous weakly nonlinear geometric optics for surface waves. Asymptot. Anal., 69(3-4):125-174, 2010. 
[Mét00] G. Métivier. The block structure condition for symmetric hyperbolic systems. Bull. London Math. Soc., 32(6):689-702, 2000.

[MR83] A. Majda and R. Rosales. A theory for spontaneous Mach stem formation in reacting shock fronts. I. The basic perturbation analysis. SIAM J. Appl. Math., 43(6):1310-1334, 1983.

[Rau12] J. Rauch. Hyperbolic partial differential equations and geometric optics, volume 133 of Graduate Studies in Mathematics. American Mathematical Society, Providence, RI, 2012.

[SS75] L. Sarason and J. A. Smoller. Geometrical optics and the corner problem. Arch. Rational Mech. Anal., 56:34-69, 1974/75.

[ST88] M. Sablé-Tougeron. Existence pour un problème de l'élastodynamique Neumann non linéaire en dimension 2. Arch. Rational Mech. Anal., 101(3):261-292, 1988. 